Nuclear Physics B161 (1979) 171-237

c) North-Holland Publishing Company

\title{
GENERAL THEORY OF VIRTUAL HEAVY-PARTICLE EFFECTS IN RENORMALIZABLE FIELD THEORIES (I) *
}

\author{
Choonkyu LEE \\ Department of Physics, The University of Michigan, Ann Arbor, Michigan 48109, USA
}

Received 21 May 1979

(Revised 23 July 1979)

We develop a systematic method of isolating the effects of virtual heavy particles in renormalizable field theories. With a $\phi^{4}$-type field-theory model involving two real scalar fields (one with a heavy mass $M$, and the other light), we show in detail that, up to order $1 / M^{2}$ (but to all orders in renormalized couplings), effects of virtual heavy particles can be completely incorporated into pure light-particle theory via effective local vertices which involve operators of canonical dimension at most six. All the coupling strengths for such effective local interactions are of order $1 / M^{2}$ (the decoupling theorem) and are systematically calculable in renormalized perturbation theory. We also derive a closed set of Callan-Symanzik equations which are satisfied by these coupling strengths. Using these equations, we explicitly sum all the leading logarithms (i.e., $g \log M \sim \mathrm{O}(1)$ ) which appear in the perturbative calculations of the effective coupling strengths.

\section{Introduction}

In field-theoretic studies of strong interaction physics, there has been much interest recently in isolating genuine large-momentum processes from complicated longdistance dynamics like confinement - so called factorization. This has been studied in terms of the operator product expansion [1] or from the viewpoint of mass singularity cancellation [2]. In the language of the former, genuine large-momentum processes are described by the so-called coefficient functions, and long-distance dynamics by the matrix elements of various local operators. In an asymptotically free field theory like QCD, the coefficient functions may be reliably calculated through perturbation theory and further improved by using the renormalization group [3]. In this paper, we discuss another kind of factorization: heavy particles versus light particles. Specifically, we wish to calculate systematically effects of virtual heavy particles on light-particle Green functions in renormalizable field theories, when all the external momenta are much smaller than the masses of heavy particles.

In renormalizable field theories without spontaneous symmetry breaking, Appel-

^ Supported in part by the Department of Energy. 
quist and Carazzone [4] have shown that the heavy particle (of mass $M$ ) decouples from light-particle physics, except for renormalization effects and corrections of order $1 / M^{2}$ (the decoupling theorem). Since renormalization effects are absorbed into the very definitions of physical parameters of the theory, this theorem practically denies any observable effects due to the existence of virtual heavy particles, to the zeroth order in $M^{-1}$. However, we do want to understand the low-energy manifestations of the heavy particles: for instance, effects of heavy muon loops in QED, the influence of heavy quarks in total $\mathrm{e}^{+} \mathrm{e}^{-}$annihilation, and, in fact, the whole subject of the weak interaction. These are order $1 / M^{2}$ effects and, in this paper, we present the precise theoretical framework to study them systematically.

We develop a simple theoretical formalism to isolate all the order $1 / M^{2}$ effects via a set of effective local vertices with calculable coupling strengths. Thus, let $m$ denote the physical light masses and $g$ the coupling strengths among these light objects, defined at appropriate normalization points. Let $M$ be the heavy masses and $G$ be the coupling strengths which involve at least one of the heavy particles. Let $T_{n}(g, m, G, M)$ be the amputated $n$ light-particle Green functions. Similarly, let $\widetilde{T}_{n}(g, m)$ be the same Green functions calculated with all heavy-particle lines taken out. We will show that, to order $1 / M^{2}$, we have the factorization:

$$
T_{n}(g, m, G, M)=\tilde{T}_{n}(g, m)+\frac{1}{M^{2}} \sum_{i} C_{i}(g, m, G, M) \widetilde{T}_{n}\left(\tilde{O}_{i}, g, m\right),
$$

where $C_{i}(g, m, G, M)$ are universal coupling strengths which can be systematically calculated in renormalized perturbation theory. $\widetilde{O}_{i}$ are local vertices involving operators made up of the light-particle fields alone and with canonical dimension at most six. $\widetilde{T}_{n}\left(\widetilde{O}_{i}, g, m\right)$ denote amputated Green functions with $\widetilde{O}_{i}$ once inserted, in the pure light-particle theory.

In renormalized perturbation theory, the effective coupling strengths $C_{i}(g, m, G$. $M)$ are at most of order $\left(\log \left(M^{2} / m^{2}\right)\right)^{n}(n$; a non-negative integer $)$, with $n$ in general increasing as the number of loops in relevant Feynman diagrams increases. It is often desirable to sum such powers of $\log \left(M^{2} / \mathrm{m}^{2}\right)$ in a systematic fashion. This can be most easily achieved if one can write down a set of closed Callan-Symanzik equations [5] for $C_{i}(g, m, G, M)$. However, the situation here is more complicated, compared to that in previously known uses of the Callan-Symanzik equations. Complications are due to the facts that:

(i) the usual right-hand sides (up to twice light-mass insertions) of the CallanSymanzik equations must not be neglected;

(ii) the Callan-Symanzik coefficients $\beta(g), \gamma(g)$, etc., also obtain $\mathrm{O}\left(1 / M^{2}\right)$ contributions from virtual heavy particles.

We have resolved these difficulties by using the homogeneous Callan-Symanzik equations developed by the author [6].

To present the main theoretical ideas without worrying too much about technical complications (like the consideration of local gauge invariance), in the present paper we have chosen a simple renormalizable field theory to prove the above state- 
ments: a $\phi^{4}$-type field theory involving two real scalar fields, one with a heavy mass $M$ and the other light. Extension to realistic field theories (e.g., QED with a heavy muon or QCD with an extra heavy quark) and physical applications will be dealt with in forthcoming publications. With our formalism, we are now able to study systematically the influence of heavy quarks in $\mathrm{e}^{+} \mathrm{e}^{-}$annihilation (or in deep inelastic scattering processes) when the incident energy is much below their production thresholds. We hope that after some extensions to incorporate spontaneously broken theories, we may be able to express arbitrary higher-order effects of virtual heavy particles in unified or grand-unified models, in terms of simple effective local vertices of the corresponding light-particle theory alone *

The plan of our paper is as follows. In sect. 2, we derive the factorization (1). In sect. 3 , we derive the Callan-Symanzik equations satisfied by the effective coupling strengths. In sect. 4, we present explicit lower-order calculations and explain how the complete leading log sum can be obtained from these calculations by using the CallanSymanzik equations. In sect. 5 , we summarize and discuss our results. Brief discussions on more realistic field theories will be also given here to give a rough idea on the scope of physical applications of our formalism. In appendix A, we discuss the factorization analogous to eq. (1) for the heavy-particle two-point function, and also for the four-point function with two external heavy particles and two external light particles. These results are used in sect. 3. In appendix B, we briefly indicate necessary modifications when different renormalization prescriptions, other than the ones used in the main text, are employed.

\section{Factorization}

We consider a scalar field theory (the $\phi-\psi$ theory) which involves two real scalar fields, $\phi$ and $\psi$, interacting via quartic couplings. The action for the system may be written as

$$
\begin{aligned}
S= & \int \mathrm{d}^{4} x\left[\frac{1}{2} \rho_{\phi}\left(\partial_{\mu} \phi\right)^{2}+\frac{1}{2} \rho_{\psi}\left(\partial_{\mu} \psi\right)^{2}-\frac{1}{2} \mu^{2} \rho_{\phi} \phi^{2}-\frac{1}{2} M^{2} \rho_{\psi} \psi^{2}-\frac{1}{4 !} \alpha \rho_{\phi}^{2} \phi^{4}\right. \\
& \left.-\frac{1}{4 !} \beta \rho_{\psi}^{2} \psi^{2}-\frac{1}{4} \gamma \rho_{\phi} \rho_{\psi} \phi^{2} \psi^{2}+\mathcal{L}_{\mathrm{c}}\right],
\end{aligned}
$$

where $(\mu, M),\left(\rho_{\phi}, \rho_{\psi}\right)$ and $(\alpha, \beta, \gamma)$ denote renormalized masses, renormalized wavefunction parameters, and renormalized coupling constants, respectively. Here, $\mathcal{L}_{\mathrm{c}}$ denotes renormalization counterterms. Since the lagrangian counterterms $\mathcal{L}_{\mathrm{c}}$ consist of local operators of canonical dimension at most four, the action (2) may also

\footnotetext{
* Some interesting papers which deal with related problems may be found in refs. [12,13]. Brief discussions are also given in sect. 5 .
} 
be written in the following multiplicative form

$$
\begin{aligned}
S= & \int \mathrm{d}^{4} x\left[\frac{1}{2} \rho_{\mathrm{B} \phi}\left(\partial_{\mu} \phi\right)^{2}+\frac{1}{2} \rho_{\mathrm{B} \psi}\left(\partial_{\mu} \psi\right)^{2}-\frac{1}{2} \mu_{\mathrm{B}}^{2} \rho_{\mathrm{B} \phi} \phi^{2}-\frac{1}{2} M_{\mathrm{B}}^{2} \rho_{\mathrm{B} \psi} \psi^{2}\right. \\
& \left.-\frac{1}{4 !} \alpha_{\mathrm{B}} \rho_{\mathrm{B} \phi}^{2} \phi^{4}-\frac{1}{4 !} \beta_{\mathrm{B}} \rho_{\mathrm{B} \psi}^{2} \psi^{4}-\frac{1}{4} \gamma_{\mathrm{B}} \rho_{\mathrm{B} \phi} \rho_{\mathrm{B} \psi} \phi^{2} \psi^{2}\right]
\end{aligned}
$$

where $\left(\mu_{\mathrm{B}}, M_{\mathrm{B}}\right),\left(\rho_{\mathrm{B} \phi}, \rho_{\mathrm{B} \psi}\right)$ and $\left(\alpha_{\mathrm{B}}, \beta_{\mathrm{B}}, \gamma_{\mathrm{B}}\right)$ denote bare masses, bare wave-function parameters, and bare coupling constants, respectively. We assume that the mass of $\psi$-particle, $M$, is much larger than the mass of $\phi$-particle, $\mu$. In this limit, we are interested in calculating effects of virtual heavy particles, here described by $\psi$, on light-particle Green functions with external momenta much smaller than $\boldsymbol{M}$.

We denote the renormalized $(n+N)$-point proper vertex function with $n$ external $\phi$-legs and $N$ external $\psi$-legs by $\Gamma_{n, N}\left(p_{1}, \ldots, p_{n}, P_{1}, \ldots, P_{N}\right)$. (See fig. 1). As our convention, dotted lines will be used to indicate $\phi$-lines, and solid lines to indicate $\psi$-lines throughout the paper. Also, all the external momenta will be specified with respect to incoming directions. Then, the renormalization counterterms in the action (2) may be chosen such that the following normalization conditions are satisfied:

$$
\begin{aligned}
& \left.\Gamma_{2,0}(p,-p)\right|_{p^{2}=0}=-i \mu^{2} \rho_{\phi}, \\
& \frac{\partial}{\partial p^{2}} \Gamma_{2,0}(p,-p) \underset{p^{2}=0}{\mid}=i \rho_{\phi}, \\
& \Gamma_{0,2}(P,-P) \underset{P^{2}=M^{2}}{\mid}=0, \\
& \frac{\partial}{\partial P^{2}} \Gamma_{0,2}(P,-P) \underset{P^{2}=M^{2}}{\mid}=i \rho_{\phi}, \\
& \Gamma_{4,0}\left(p_{1}, p_{2}, p_{3}, p_{4}\right) \underset{p_{i}=0}{\mid}=-i \alpha \rho_{\phi}^{2}, \\
& \Gamma_{0,4}\left(P_{1}, P_{2}, P_{3}, P_{4}\right) \quad=\quad P_{i}^{2}=M^{2} \quad=-i \beta \rho_{\psi}^{2}, \\
& \left(P_{1}+P_{2}\right)^{2}=\left(P_{2}+P_{3}\right)^{2}=\frac{4}{3} M^{2} \\
& \Gamma_{2,2}\left(p_{1}, p_{2},\right)_{p_{i}=0, P_{1}^{2}=P_{2}^{2}=M^{2}}^{\mid}=-i \gamma \rho_{\phi} \rho_{\psi} .
\end{aligned}
$$

Precise details of these normalization conditions are not important, as long as one chooses the normalization points for light particles (i.e., $\phi$-fields) at momenta much smaller than $M$. (The latter is necessary for the decoupling theorem to be valid in 


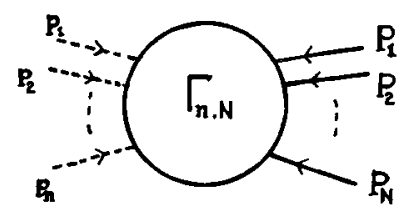

Fig. 1. $\mathrm{A}(n+N)$-point proper vertex function.

the first place). Also, in eqs. $(4 a-g)$ we renormalized the theory at zero momenta for external $\phi$-fields, only for the sake of simplicity. In appendix B, cases with different normalization conditions will be considered.

We now address ourselves the following question: For the function $\Gamma_{n, 0}\left(p_{1}, \ldots, p_{n}\right)$ in the limit of $\mu, p_{1}, \ldots, p_{n}<<M$, is it possible to describe the effects of virtual heavy particles in terms of some simple quantities of pure light-particle theory? According to the decoupling theorem [4], the effects are in general of order $1 / M^{2}$. But, do these order $1 / M^{2}$ effects appear in a certain systematic fashion? To be more precise, let us consider a theory of light particles (the $\phi$ theory) with the action

$$
\tilde{S}=\int \mathrm{d}^{4} x\left\{\frac{1}{2} \rho_{\phi}\left(\partial_{\mu} \phi\right)^{2}-\frac{1}{2} \mu^{2} \rho_{\phi}^{2} \phi^{2}-\frac{1}{4 !} \alpha \rho_{\phi}^{2} \phi^{4}+\widetilde{\mathcal{L}}_{\mathrm{c}}\right\},
$$

or, in a multiplicative form,

$$
\tilde{S}=\int \mathrm{d}^{4} x\left\{\frac{1}{2} \tilde{\rho}_{\mathrm{B} \phi}\left(\partial_{\mu} \phi\right)^{2}-\frac{1}{2} \tilde{\mu}_{\mathrm{B}}^{2} \tilde{\rho}_{\mathrm{B} \phi}^{2} \phi^{2}-\frac{1}{4 !} \tilde{\alpha}_{\mathrm{B}} \tilde{\rho}_{\mathrm{B} \phi}^{2} \phi^{4}\right\} .
$$

Denoting the $n$-point proper vertex function in this theory by $\tilde{\Gamma}_{n}\left(p_{1}, \ldots, p_{n}\right)$, we may impose the following normalization conditions:

$$
\begin{aligned}
& \left.\widetilde{\Gamma}_{2}(p,-p)\right|_{p^{2}=0}=-i \mu^{2} \rho_{\phi}, \\
& \frac{\partial}{\partial p^{2}} \widetilde{\Gamma}_{2}(p,-p) \underset{p^{2}=0}{\mid}=i \rho_{\phi}, \\
& \tilde{\Gamma}_{4}\left(p_{1}, p_{2}, p_{3}, p_{4}\right) \underset{p_{i}=0}{\mid}=-i \alpha \rho_{\phi}^{2},
\end{aligned}
$$

namely the same values as those in the $\phi-\psi$ theory with the action (2) at the common normalization points. (See the conditions (4a), (4b), and (4e)). Here, we have assigned the same values for observed (i.e., renormalized) parameters in both theories since renormalization effects are not directly observable. (This also implies that

$$
\left.\tilde{\mathcal{L}}_{\mathrm{c}} \neq \tilde{\mathscr{L}}_{\mathrm{c}}, \quad \rho_{\mathrm{B} \phi} \neq \tilde{\rho}_{\mathrm{B} \phi}, \quad \mu_{\mathrm{B}}^{2} \neq \tilde{\mu}_{\mathrm{B}}^{2}, \quad \alpha_{\mathrm{B}} \neq \tilde{\alpha}_{\mathrm{B}}\right) .
$$




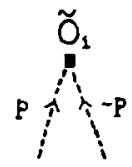

$P_{\phi}\left(p^{2}\right)^{2}$

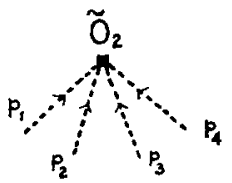

$\rho_{\phi}^{2}\left(p_{1}^{2}+p_{2}^{2}+p_{3}^{2}+p_{4}^{2}\right)$

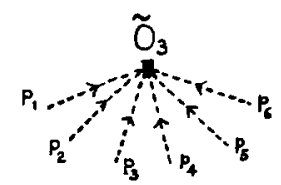

$\rho_{\phi}^{3}$

Fig. 2. Tree vertices for $\widetilde{O}_{1}, \widetilde{O}_{2}, \widetilde{O}_{3}$.

We now introduce the function

$$
H_{n}\left(p_{1}, \ldots, p_{n}\right)=\Gamma_{n, 0}\left(p_{1}, \ldots, p_{n}\right)-\tilde{\Gamma}_{n}\left(p_{1}, \ldots, p_{n}\right),
$$

and then the question we have just addressed ourselves may be put as follows: Is there any systematic method of expressing $H_{n}\left(p_{1}, \ldots, p_{n}\right)$ in terms of some simple quantities of the pure light-particle theory only? To all orders in renormalized perturbation theory, we will now show that, for $\mu, p_{1}, \ldots, p_{n}<<M^{\star}$,

$$
H_{n}\left(p_{1}, \ldots, p_{n}\right)=\sum_{i} G_{i} \tilde{\Gamma}_{n}\left(\tilde{O}_{i}, p_{1}, \ldots, p_{n}\right)+\mathrm{O}\left(\frac{1}{M^{4}}\right),
$$

where the $G_{i}$ 's are of order $1 / M^{2}$ and $\widetilde{O}_{i}$ denotes certain local vertices in the $\phi$-field theory as specified below.

In $\widetilde{O}_{i}$, one should in general include all the local vertices involving operators of canonical dimension not larger than six. However, with the normalization conditions $(4 \mathrm{a}-\mathrm{g}),(7 \mathrm{a}-\mathrm{c})$ and the normalization conditions for $\widetilde{O}_{i}$ 's as specified below, the most convenient set of the local vertices are provided by the following vertices which involve only dimension-six operators,

$$
\begin{aligned}
& \widetilde{O}_{1}=\frac{\rho_{\phi}}{2} \int \mathrm{d}^{4} x N\left[\phi\left(\partial^{2}\right)^{2} \phi(x)\right], \\
& \widetilde{O}_{2}=-\frac{\rho_{\phi}^{2}}{6} \int \mathrm{d}^{4} x N\left[\phi^{3} \partial^{2} \phi(x)\right], \\
& \widetilde{O}_{3}=\frac{\rho_{\phi}^{3}}{6 !} \int \mathrm{d}^{4} x N\left[\phi^{6}(x)\right] .
\end{aligned}
$$

Precise definitions of these local vertices can be given (traditionally in momentum space) by specifying the corresponding tree vertices and appropriate subtraction rules in calculating higher-order matrix elements involving these new vertices. The tree vertices are shown in fig. 2. Assuming minimum subtractions (i.e., subtract only for the purpose of making certain divergent subgraphs finite and no further

* Here, the notation $p_{1}, \ldots, p_{n}<<M$ implies that every individual component of the four-momenta $p_{1}, \ldots, p_{n}$ is much smaller than $M$. 
finite renormalizations), the subtraction rules for the above operators can be completely specified by the following normalization conditions:

$$
\begin{aligned}
& \left.\widetilde{\Gamma}_{2}\left(\widetilde{O}_{i}, p,-p\right)\right|_{p^{2}=0}=0, \quad(i=1,2,3), \\
& \left.\frac{\partial}{\partial p^{2}} \tilde{\Gamma}_{2}\left(\tilde{O}_{i}, p,-p\right)\right|_{p^{2}=0}=0, \quad(i=1,2,3), \\
& \left.\tilde{\Gamma}_{4}\left(\tilde{O}_{i}, p_{1}, p_{2}, p_{3}, p_{4}\right)\right|_{p_{i}=0}=0, \quad(i=1,2,3), \\
& \widetilde{\Gamma}_{2}\left(\tilde{O}_{1}, p,-p\right) \underset{p^{2} \rightarrow 0}{=} \rho_{\phi}\left[\left(p^{2}\right)^{2}+\mathrm{O}\left(\left(p^{2}\right)^{3}\right)\right] \\
& \widetilde{\Gamma}_{4}\left(\tilde{O}_{1}, p_{1}, p_{2}, p_{3}, p_{4}\right) \underset{p_{i} \rightarrow 0}{=} \mathrm{O}\left(p_{i}^{4}\right) \\
& \widetilde{\Gamma}_{6}\left(\tilde{O}_{1}, p_{1}, p_{2}, \ldots, p_{6}\right) \underset{p_{i} \rightarrow 0}{=} \mathrm{O}\left(p_{i}^{2}\right) \\
& \tilde{\Gamma}_{2}\left(\tilde{O}_{2}, p,-p\right) \underset{p^{2} \rightarrow 0}{=} \mathrm{O}\left(\left(p^{2}\right)^{3}\right) \\
& \widetilde{\Gamma}_{4}\left(\widetilde{O}_{2}, p_{1}, p_{2}, p_{3}, p_{4}\right) \underset{p_{i} \rightarrow 0}{=} \rho_{\phi}^{2}\left[p_{1}^{2}+p_{2}^{2}+p_{3}^{2}+p_{4}^{2}\right]+\mathrm{O}\left(p_{i}^{4}\right) \\
& \widetilde{\Gamma}_{6}\left(\widetilde{O}_{2}, f_{1}, \ldots, p_{6}\right) \underset{p_{i} \rightarrow 0}{=} \mathrm{O}\left(p_{i}^{2}\right) \\
& \widetilde{\Gamma}_{2}\left(\tilde{O}_{3}, p,-p\right) \underset{p^{2} \rightarrow 0}{=} \mathrm{O}\left(\left(p^{2}\right)^{3}\right) \\
& \widetilde{\Gamma}_{4}\left(\tilde{O}_{3}, p_{1}, p_{2}, p_{3}, p_{4}\right) \underset{p_{i} \rightarrow 0}{=} \mathrm{O}\left(p_{i}^{4}\right), \\
& \widetilde{\Gamma}_{6}\left(\widetilde{O}_{3}, p_{1}, \ldots, p_{6}\right) \underset{p_{i} \rightarrow 0}{=} \rho_{\phi}^{3}+\mathrm{O}\left(p_{i}^{2}\right) .
\end{aligned}
$$

The normalization conditions $(11 \mathrm{a}-\mathrm{c})$ are for the subtraction of quartic and quadratic divergences and, as will be seen later, are closely related to original renormalization subtractions in the $\phi-\psi$ theory with the action (2).

To prove the factorization (9), it is convenient to adopt the elegant language due to Zimmermann in defining renormalization subtractions [7]. But, in the present paper, we use a slight deviation from Zimmerman's original approach to facilitate our discussions.

Let us first look at the $\phi-\psi$ theory. The action (2) with $\mathcal{L}_{\mathrm{c}}=0$ provides Feyn- 


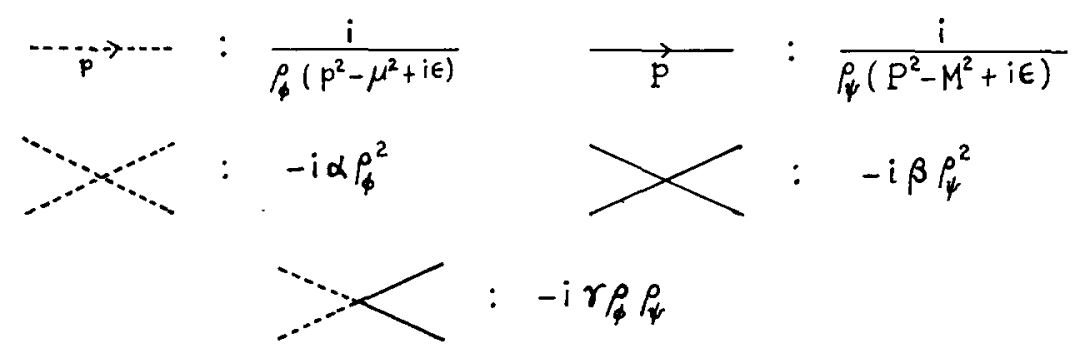

1ig. 3. Feyman rules for the $\phi-\psi$ theory.

man rules for unrenormalized Green functions, with the rules for tree graphs shown in fig. 3. Higher-order, unrenormalized, Green functions are expressed in terms of the tree-graph Feynman rules by using the Gell-Mann-Low theorem and Wick theorem [8]. The proper vertex function $\Gamma_{n, N}^{u}\left(p_{1}, \ldots, p_{n}, P_{1}, \ldots, P_{N}\right)$ is in general illdefined due to ultraviolet divergence problems. To make them well-defined, we may regulate those unsubtracted functions by well-known regularization methods, either by the Pauli-Villars regularization or by the dimensional continuation method. To be definite, let us take the Pauli-Villars method with the typical regulator mass denoted by $\Lambda$. Then the renormalized function $\Gamma_{n, N}\left(p_{1}, \ldots, p_{n}, P_{1}, \ldots, P_{N}\right)$ is given by the forest formula [7]

$$
\Gamma_{n, N}\left(p_{1}, \ldots, p_{n}, P_{1}, \ldots, P_{N}\right)=\lim _{\Lambda^{2} \rightarrow \infty} \sum_{\Omega} \prod_{\gamma \in \Omega}\left(-t_{\gamma}^{(0)}\right) \Gamma_{n, N}^{\mathrm{u}}\left(p_{1} \ldots, p_{n}, P_{1}, \ldots, P_{N}\right),
$$

where the sum goes over all the forests of each Feynman graph contributing to $\Gamma_{n, N}^{\mathrm{u}}$.

Let us explain the formula (12) briefly. A forest $\Omega$ is defined as a set of non-overlapping proper subgraphs, $\gamma$, of a given Feynman graph G. (Note that Zimmermann [7] defines a forest in terms of non-overlapping renormalization parts, rather than in terms of arbitrary non-overlapping proper subgraphs. Instead, we will put some restrictions upon the Taylor operator $t_{\gamma}^{(0)}$ ). Thus, any two elements $\gamma, \gamma^{\prime}$ in a forest satisfy either $\gamma \subset \gamma^{\prime}$ or $\gamma \supset \gamma^{\prime}$ or $\gamma \cap \gamma^{\prime}=0$. A forest $\Omega$ containing the whole graph $\mathrm{G}$ itself is callel full, and a forest not containing the whole graph is called normal. A proper subgraph $\gamma$ is defined by a non-empty set of lines of $\mathrm{G}$ which are connected and one-particle-irreducible. According to this definition, the empty set or a trivial diagram consisting of a single vertex and no line is not a proper subgraph. On the other hand, the one-particle-irreducible whole graph $\mathrm{G}$ is considered as a proper subgraph if $\mathrm{G}$ does not correspond to a tree graph. Also, $\Omega$ may be empty set, and it is important to include this empty set in the sum over forests in eq. (12). The Taylor operator $t_{\gamma}^{(0)}$ operates on the part of the integrand which precisely represents the proper subgraph $\gamma$. (The superscript '(0)' in $t_{\gamma}^{(0)}$ indicates minimal or normal subtractions). Let the proper subgraph $\gamma$ correspond to a $(k+K)$-point vertex function with the external momenta $q_{1}, \ldots, q_{k}, Q_{1}, \ldots, Q_{K}$, and then we may denote the cor- 


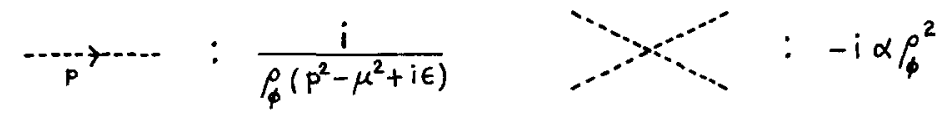

Fig. 4. Feynman rules for the $\phi$ theory.

responding Feynman amplitude simply by $\Gamma_{k, K}^{\mathrm{u}}\left(q_{1}, \ldots, q_{k}, Q_{1}, \ldots, Q_{K}\right)$ in a representative sense. Then the Taylor operator $t_{\gamma}^{(0)}$ is specified by

$$
\begin{aligned}
& t^{(0)} \Gamma_{2,0}^{u}(q,-q)=\Gamma_{2,0}^{\mathrm{u}}(q,-q) \underset{q^{2}=0}{\mid}+q^{2}\left[\frac{\partial}{\partial q^{2}} \Gamma_{2,0}^{\mathrm{u}}(q,-q)\right]_{q^{2}=0} \\
& t^{(0)} \Gamma_{4,0}^{\mathrm{u}}\left(q_{1}, q_{2}, q_{3}, q_{4}\right)=\Gamma_{4,0}^{\mathrm{u}}\left(q_{1}, q_{2}, q_{3}, q_{4}\right) \underset{q_{i}=0}{1}, \\
& t^{(0)} \Gamma_{0,2}^{\mathrm{u}}(Q,-Q)=\Gamma_{0,2}^{\mathrm{u}}(Q,-Q) \underset{Q^{2}=M^{2}}{\mid}+\left(Q^{2}-M^{2}\right)\left[\frac{\partial}{\partial Q^{2}} \Gamma_{0,2}^{\mathrm{u}}(Q,-Q)\right]_{Q^{2}=M^{2}}^{\mid}, \\
& t^{(0)} \Gamma_{0,4}^{\mathrm{u}}\left(Q_{1}, Q_{2}, Q_{3}, Q_{4}\right)=\Gamma_{0,4}^{\mathrm{u}}\left(Q_{1}, Q_{2}, Q_{3}, Q_{4}\right) \\
& \begin{array}{c}
Q_{i}^{2}=M^{2} \\
\left(Q_{1}+Q_{2}\right)^{2}=\left(Q_{2}+Q_{3}\right)^{2}=\left(Q_{1}+Q_{3}\right)^{2}=\frac{4}{3} M^{2}
\end{array} \\
& t^{(0)} \Gamma_{2,2}^{\mathrm{u}}\left(q_{1}, q_{2}, Q_{1}, Q_{2}\right)=\Gamma_{2,2}^{\mathrm{u}}\left(q_{1}, q_{2}, Q_{1}, Q_{2}\right) \underset{q_{i}=0, Q_{1}^{2}=Q_{2}^{2}=M^{2}}{\mid}, \\
& t^{(0)} \Gamma_{k, K}^{\mathrm{u}}\left(q_{1}, \ldots, q_{k}, Q_{1}, \ldots, Q_{K}\right)=0, \quad(k+K>4) .
\end{aligned}
$$

In the product $\Pi\left(-t_{\gamma}^{(0)}\right)$ in the forest formula $(12)$, the factors are ordered such that $t_{\gamma}^{(0)}$ stands to the left of $t_{\gamma^{\prime}}^{(0)}$, if $\gamma \supset \gamma^{\prime}$. For the empty forest, the product $\Pi\left(-t_{\gamma}^{(0)}\right)$ is simply reduced to 1 . With these prescriptions, one can show that the expression (12) has a well-defined limit and satisfies the normalization conditions $(4 \mathrm{a}-\mathrm{g})$. It is also matter of combinatorics to show that these renormalization subtractions correspond to multiplicative renormalizations, as are manifest in the second form of the action, eq. (3).

Almost parallel considerations may be given for the $\phi$-field theory with the action (5). Tree-graph rules for unsubtracted Green functions are shown in fig. 4 . The renormalized proper vertex function $\widetilde{\Gamma}_{n}\left(p_{1}, \ldots, p_{n}\right)$ in this theory is given by the forest formula

$$
\tilde{\Gamma}_{n}\left(p_{1}, \ldots, p_{n}\right)=\lim _{\Lambda^{2} \rightarrow \infty} \sum_{\Omega} \prod_{\gamma \in \Omega}\left(-t_{\gamma}^{(0)}\right) \tilde{\Gamma}_{n}^{u}\left(p_{1}, \ldots, p_{n}\right)
$$


with the Taylor operator $t_{\gamma}^{(0)}$ specified by

$$
\begin{aligned}
& t^{(0)} \widetilde{\Gamma}_{2}^{\mathrm{u}}(q,-q)=\widetilde{\Gamma}_{2}^{\mathrm{u}}(q,-q) \underset{q^{2}=0}{\mid}+q^{2}\left[\frac{\partial}{\partial q^{2}} \tilde{\Gamma}_{2}^{\mathrm{u}}(q,-q)\right]_{q^{2}=0}, \\
& t^{(0)} \widetilde{\Gamma}_{4}^{\mathrm{u}}\left(q_{1}, q_{2}, q_{3}, q_{4}\right)=\widetilde{\Gamma}_{4}^{\mathrm{u}}\left(q_{1}, q_{2}, q_{3}, q_{4}\right) \underset{q_{i}=0}{\mid,}, \\
& t^{(0)} \widetilde{\Gamma}_{k}^{\mathrm{u}}\left(q_{1}, \ldots, q_{k}\right)=0, \quad(k>4) .
\end{aligned}
$$

The renormalized functions $\widetilde{\Gamma}_{n}$ defined by the formula (14) satisfy the normalization conditions $(7 \mathrm{a}-\mathrm{c})$. Also, renormalized proper vertex functions with a certain operator vertex insertion may be defined in an analogous manner, if the tree-graph rule for the operator vertex to calculate unsubtracted functions has been given. In particular, the renormalized vertex functions $\widetilde{\Gamma}_{n}\left(\widetilde{O}_{i}, p_{1}, \ldots, p_{n}\right)$ with the operator vertices $\widetilde{O}_{i}$ (see fig. 2 for the tree-graph rules) are given by the forest formulae

$$
\tilde{\Gamma}_{n}\left(\tilde{O}_{i}, p_{1}, \ldots, p_{n}\right)=\lim _{\Lambda^{2} \rightarrow \infty} \sum_{\Omega} \prod_{\gamma \in \Omega}\left(-t_{\gamma}^{(0)}\right) \widetilde{\Gamma}_{n}^{u}\left(\widetilde{O}_{i}, p_{1}, \ldots, p_{n}\right)
$$

with the Taylor operator $t_{\gamma}^{(0)}$ specified as follows:

(i) If the proper subgraph $\gamma$ corresponds to a vertex function which does not include the $\widetilde{O}_{i}$ vertex, $t_{\gamma}^{(0)}$ is given by eqs. $(15 \mathrm{a}-\mathrm{c})$.

(ii) If the proper subgraph $\gamma$ includes the $\widetilde{O}_{i}$ vertex, $t_{\gamma}^{(0)}$ is determined by

$$
\begin{aligned}
& t^{(0)} \widetilde{\Gamma}_{2}^{\mathrm{u}}\left(\widetilde{O}_{i}, q,-q\right)=\widetilde{\Gamma}_{2}^{\mathrm{u}}\left(\widetilde{O}_{i}, q,-q\right) \underset{q^{2}=0}{\mid}+q^{2} \frac{\partial}{\partial q^{2}} \widetilde{\Gamma}_{2}^{\mathrm{u}}\left(\tilde{O}_{i}, q,-q\right) \underset{q^{2}=0}{\mid} \\
& +\frac{1}{2}\left(q^{2}\right)^{2}\left[\frac{\partial^{2}}{\partial\left(q^{2}\right)^{2}} \widetilde{\Gamma}_{2}^{u}\left(\widetilde{Q}_{i}, q,-q\right)\right]_{q^{2}=0}^{\mid}, \\
& t^{(0)} \tilde{\Gamma}_{4}^{\mathrm{u}}\left(\widetilde{O}_{i}, q_{1}, q_{2}, q_{3}, q_{4}\right)=\widetilde{\Gamma}_{4}^{\mathrm{u}}\left(\widetilde{O}_{i}, q_{1}, \ldots, q_{4}\right) \underset{q_{i}=0}{\mid} \\
& +\left.\frac{1}{2} \sum_{k \neq l} q_{k} q_{l}\left[\frac{\partial^{2}}{\partial q_{k} \partial q_{l}} \widetilde{\Gamma}_{4}^{\mathrm{u}}\left(\widetilde{O}_{i}, q_{1}, \ldots, q_{4}\right)\right]\right|_{q_{i}=0} \\
& +\frac{1}{2} \sum_{k} q_{k}^{2}\left[\frac{\partial^{2}}{\partial q_{k}^{2}} \widetilde{\Gamma}_{4}\left(\tilde{O}_{i}, q_{1}, \ldots, q_{4}\right)\right]_{q_{i}=0}, \\
& t^{(0)} \widetilde{\Gamma}_{6}^{\mathrm{u}}\left(\widetilde{O}_{i}, q_{1}, \ldots, q_{6}\right)=\left.\widetilde{\Gamma}_{6}^{\mathrm{u}}\left(\widetilde{O}_{i}, q_{1}, \ldots, q_{6}\right)\right|_{q_{i}=0}, \\
& t^{(0)} \tilde{\Gamma}_{k}^{\mathrm{u}}\left(\tilde{O}_{i}, q_{1}, \ldots, q_{k}\right)=0, \quad(k>6) .
\end{aligned}
$$

(In eq. (17b), the variable $q_{k}$ may refer to any of $q_{1 \mu}, q_{2 \mu}, q_{3 \mu}, q_{4 \mu}$ with an arbitrary 
Lorentz index.) Again, it is a simple matter to show that $\widetilde{\Gamma}_{n}\left(O_{i}, p_{1}, \ldots, p_{n}\right)$ defined by the forest formula (16) satisfies the normalization conditions $(11 \mathrm{a}-\ell)$.

We are now ready to prove the factorization (9). The proof which follows is based on the adaptation of Zimmermann's algebraic identity to our problem [7]. Let us define the unsubtracted function

$$
H_{n}^{\mathrm{u}}\left(p_{1}, \ldots, p_{n}\right)=\Gamma_{n, 0}^{\mathrm{u}}\left(p_{1}, \ldots, p_{n}\right)-\widetilde{\Gamma}_{n}^{\mathrm{u}}\left(p_{1}, \ldots, p_{n}\right),
$$

which is represented by all one-particle-irreducible Feynman graphs with $n$ external $\phi$-legs and some internal $\psi$-lines. Then, the renormalized function $H_{n}\left(p_{1}, \ldots, p_{n}\right)$ as defined by eq. (8) can be given by the forest formula

$$
H_{n}\left(p_{1}, \ldots, p_{n}\right)=\sum_{\Omega} \prod_{\gamma \in \Omega}\left(-t_{\gamma}^{(0)}\right) H_{n}^{\mathrm{u}}\left(p_{1}, \ldots, p_{n}\right),
$$

where the limit $\Lambda^{2} \rightarrow \infty$ is implicitly assumed (here and henceforth) and the sum goes over all the forests in the $\phi-\psi$ theory. If $\Omega$ is not an empty forest, the elements $\gamma$ in general correspond to a certain proper vertex function, $\Gamma_{n, N}^{\mathrm{u}}$. When a proper subgraph $\gamma \in \Omega$ corresponds to $\Gamma_{n, 0}^{\mathrm{u}}$, it is convenient to separate the case when $\gamma$ includes some internal $\psi$-lines from the case when $\gamma$ includes only $\phi$-lines. Hence, for the latter case we shall say that $\gamma$ corresponds to $\widetilde{\Gamma}_{n}^{\mathrm{u}}$, and for the former case to $H_{n}^{\mathrm{u}}$. We may then say that the elements $\gamma$ with $t_{\gamma}^{(0)} \neq 0$ correspond to one of $\widetilde{\Gamma}_{2}^{\mathrm{u}}, \widetilde{\Gamma}_{4}^{\mathrm{u}}, H_{2}^{\mathrm{u}}, H_{4}^{\mathrm{u}}, \Gamma_{0.2}^{\mathrm{u}}, \Gamma_{2.2}^{\mathrm{u}}, \Gamma_{0.4}^{\mathrm{u}}$.

To prove the factorization (9), it is useful to consider the oversubtracted function $\bar{H}_{n}\left(p_{1}, \ldots, p_{n}\right)$ which may be written as

$$
\bar{H}_{n}\left(p_{1}, \ldots, p_{n}\right)=\sum_{\Omega} \prod_{\gamma \in \Omega}\left(-\bar{t}_{\gamma}\right) H_{n}^{\mathrm{u}}\left(p_{1}, \ldots, p_{n}\right) .
$$

The Taylor operator $\bar{t}_{\gamma}$ is given by

$$
\begin{array}{ll}
\bar{t}_{\gamma}=t_{\gamma}^{(2)}, \begin{array}{l}
\text { if } \gamma \text { corresponds to } H_{2}^{\mathrm{u}}, H_{4}^{\mathrm{u}} \text { or } H_{6}^{\mathrm{u}} \text { and there are } \\
\text { no internal } \psi \text {-lines left outside } \gamma,
\end{array} \\
\bar{t}_{\gamma}=t_{\gamma}^{(0)}, \quad \text { otherwise } .
\end{array}
$$

The Taylor operator $t_{\gamma}^{(2)}$ indicates oversubtraction and is precisely specified by $(c f$. eqs. $(17 \mathrm{a}-\mathrm{d}))$

$$
\begin{gathered}
t^{(2)} H_{2}^{\mathrm{u}}(q,-q)=\left.H_{2}^{\mathrm{u}}(q,-q){ }_{q^{2}=0}\right|^{2}+q^{2}\left[\frac{\partial}{\partial q^{2}} H_{2}^{\mathrm{u}}(q,-q)\right]_{q^{2}=0} \\
+\frac{1}{2}\left(q^{2}\right)^{2}\left[\frac{\partial^{2}}{\partial\left(q^{2}\right)^{2}} H_{2}^{\mathrm{u}}(q,-q)\right]_{q^{2}=0}, \\
t^{(2)} H_{4}^{\mathrm{u}}\left(q_{1}, q_{2}, q_{3}, q_{4}\right)=\left.H_{4}^{\mathrm{u}}\left(q_{1}, \ldots, q_{4}\right)\right|_{q_{i}=0}+\frac{1}{2} \sum_{k \neq l} q_{k} q_{l}\left[\frac{\partial^{2}}{\partial q_{k} \partial q_{l}} H_{4}^{\mathrm{u}}\left(q_{1}, \ldots, q_{4}\right)\right]_{q_{i}=0}
\end{gathered}
$$




$$
\begin{aligned}
& +\left.\frac{1}{2} \sum_{k} q_{k}^{2}\left[\frac{\partial^{2}}{\partial q_{k}^{2}} H_{4}^{\mathrm{u}}\left(q_{1}, \ldots, q_{4}\right)\right]\right|_{q_{i}=0}, \\
& t^{(2)} H_{6}^{\mathrm{u}}\left(q_{1}, \ldots, q_{6}\right)=H_{6}^{\mathrm{u}}\left(q_{1}, \ldots, q_{6}\right) \underset{q_{i}=0}{\mid}, \\
& t^{(2)} H_{k}^{\mathrm{u}}\left(q_{1}, \ldots, q_{k}\right)=0, \quad(k>6) .
\end{aligned}
$$

Here, for any forest, we may consider the set $\vartheta$ consisting of all the proper subgraphs satisfying the condition (21a). Then, any two elements $\gamma, \gamma^{\prime} \in \mathcal{V}$ must satisfy $\gamma^{\prime} \subset \gamma$ or $\gamma^{\prime} \supset \gamma$, since there should not be any internal $\psi$-lines left outside $\gamma\left(\right.$ or $\left.\gamma^{\prime}\right)$.

We now rearrange the formula (20). Let us first define the set of all forests $\Omega$ for a given Feynman graph as $U(\Omega)$. Then, for any forest $\Omega \in \cup(\Omega)$ which includes at least one element belonging to $\mathcal{V}$ we may split

$$
\bar{t}_{\gamma}=t_{\gamma}^{(0)}+\left(t_{\gamma}^{(2)}-t_{\gamma}^{(0)}\right), \quad \text { for any } \gamma \in \mathcal{V} \text {. }
$$

Substituting eq. (23) into eq. (20), we obtain

$$
\bar{H}_{n}\left(p_{1}, \ldots, p_{n}\right)=\sum_{\Omega \in \cup(\Omega)} \sum_{F \in \mathrm{F}(\Omega)} \prod_{\gamma \in \Omega} F_{\gamma} H_{n}^{\mathrm{u}}\left(p_{1}, \ldots, p_{n}\right),
$$

where $F(\Omega)$ is the family of the functions with the property

$$
F_{\gamma}= \begin{cases}\text { either }-t_{\gamma}^{(0)} \text { or }-\left(t_{\gamma}^{(2)}-t_{\gamma}^{(0)}\right), & \text { if } \gamma \in \mathcal{V}, \\ -t_{\gamma}^{(0)}, & \text { if } \gamma \notin \mathcal{V} .\end{cases}
$$

For any $\Omega$, there must be a function $F_{0}$ in $\mathrm{F}(\Omega)$ which assigns $-t_{\gamma}^{(0)}$ for all $\gamma \in \Omega$. Taking out all terms with $F=F_{0}$ and using the forest formula (19), we find

$$
\bar{H}_{n}\left(p_{1}, \ldots, p_{n}\right)=H_{n}\left(p_{1}, \ldots, p_{n}\right)+X_{n}\left(p_{1}, \ldots, p_{n}\right),
$$

where

$$
X_{n}\left(p_{1}, \ldots, p_{n}\right)=\sum_{\Omega \in \cup^{\prime}(\Omega)} \sum_{\substack{F \in F(\Omega) \\ F \neq F_{0}}} \prod_{\gamma \in \Omega} F_{\gamma} H_{n}^{\mathrm{u}}\left(p_{1}, \ldots, p_{n}\right),
$$

with $U^{\prime}(\Omega)$ denoting the set of all forests which have at least one element belonging to $\mathcal{V}$.

In the sum (27), we now note that, for any $\Omega$ and $F \neq F_{0}$, there is a smallest element $\tau \in \mathcal{V}$ which gets the assignment

$$
F_{\tau}=-\left(t_{\tau}^{(2)}-t_{\tau}^{(0)}\right)
$$

Thus, for given $\Omega$ and $F$, we may decompose

$$
\Omega=\Omega_{1}+\Omega_{2}+\{\tau\},
$$


where

$\Omega_{1}:$ the set of all $\gamma \in \Omega(\gamma \neq \tau)$ with $\gamma \supset \tau$ or $\gamma \cap \tau=0$,

$\Omega_{2}$ ? the set of all $\gamma \in \Omega(\gamma \neq \tau)$ with $\gamma \subset \tau$.

Note that $\Omega_{2}$ is a normal forest of $\tau$, i.e., $\Omega_{2} \in \cup(\tau)$ with $\cup(\tau)$ denoting the set of all normal forests of $\tau$. The decomposition (29) implies that, for a given forest $\Omega$,

$$
\sum_{\substack{F \in F(\Omega) \\ F \neq F_{0}}} \prod_{\gamma \in \Omega} F_{\gamma} H^{\mathfrak{u}}=\left[\prod_{\gamma \in \Omega_{1}}\left(-\bar{t}_{\gamma}\right)\right]\left[-\left(t_{\tau}^{(2)}-t_{\tau}^{(0)}\right)\right]\left[\prod_{\gamma^{\prime} \in \Omega_{2}}\left(-t_{\gamma}^{(0)}\right)\right] H^{\mathrm{u}} .
$$

With this information, it is a simple matter to write $X_{n}\left(p_{1}, \ldots, p_{n}\right)$ in the form

$$
\begin{aligned}
& \left.X_{n}\left(p_{1}, \ldots, p_{n}\right)=\sum_{\tau \in \mathrm{T}} \sum_{\Omega_{1} \in \mathrm{M}_{\tau}} \sum_{\Omega_{2} \in U(\tau)} \prod_{\gamma \in \Omega_{1}}\left(-\bar{t}_{\gamma}\right)\right] \cdot\left[-\left(t_{\tau}^{(2)}-t_{\tau}^{(0)}\right)\right] \\
& \cdot\left[\prod_{\gamma^{\prime} \in \Omega_{2}}\left(-t_{\gamma}^{(0)}\right)\right] H^{\mathrm{u}},
\end{aligned}
$$

where

$\mathrm{T}$ : the set of all proper subgraphs $\tau$ having the property that $\tau$ corresponds to $H_{2}^{\mathrm{u}}, H_{4}^{\mathrm{u}}$, or $H_{6}^{\mathrm{u}}$ and there are no internal $\psi$-lines left outside $\tau$,

$\mathrm{M}_{\tau}$ : the set of forests $\Omega_{1}$ having the property that each $\gamma \in \Omega_{1}$ satisfies $\tau \subset \gamma$ or $\tau \cap \gamma=0\left(\mathrm{M}_{\tau}\right.$ may be empty),

$\cup(\tau)$ : the set of all normal forests of $\tau$.

From eqs. (26) and (31), we conclude that

$$
\begin{aligned}
& H_{n}\left(p_{1}, \ldots, p_{n}\right)=-\sum_{\tau \in \mathrm{T}} \sum_{\Omega_{1} \in \mathrm{M}_{\tau}} \sum_{\Omega_{2} \in U(\tau)}\left[\prod_{\gamma \in \Omega_{1}}\left(-\bar{t}_{\gamma}\right)\right] \cdot\left[-\left(t_{\tau}^{(2)}-t_{\tau}^{(0)}\right)\right] \\
& \cdot\left[\prod_{\gamma^{\prime} \in \Omega_{2}}\left(-t_{\gamma}^{(0)}\right)\right] H_{n}^{\mathrm{u}}\left(p_{1}, \ldots, p_{n}\right)+\bar{H}_{n}\left(p_{1}, \ldots, p_{n}\right) .
\end{aligned}
$$

In the expression (31) for $X_{n}\left(p_{1}, \ldots, p_{n}\right)$, we note that all the internal $\psi$-lines are inside the proper subgraph $\tau$. Then, we may define the constants $G_{1}, G_{2}, G_{3}$ by

$$
\begin{gathered}
\left(t^{(2)}-t^{(0)}\right) \sum_{\Omega}^{\text {normal }} \prod_{\gamma \in \Omega}\left(-t_{\gamma}^{(0)}\right) H_{2}^{\mathrm{u}}(q,-q)=\rho_{\phi}\left(q^{2}\right)^{2} G_{1}, \\
\left(t^{(2)}-t^{(0)}\right) \sum_{\Omega}^{\text {normal }} \prod_{\gamma \in \Omega}\left(-t_{\gamma}^{(0)}\right) H_{4}^{\mathrm{u}}\left(q_{1}, q_{2}, q_{3}, q_{4}\right)=\rho_{\phi}^{2}\left(q_{1}^{2}+q_{2}^{2}+q_{3}^{2}+q_{4}^{2}\right) G_{2}, \\
\left(t^{(2)}-t^{(0)}\right) \sum_{\Omega}^{\text {normal }} \prod_{\gamma \in \Omega}\left(-t_{\gamma}^{(0)}\right) H_{6}^{\mathrm{u}}\left(q_{1}, \ldots, q_{6}\right)=\rho_{\phi}^{3} G_{3},
\end{gathered}
$$

where $\Sigma_{\Omega}^{\text {normal }}$ indicates the sum over normal forests only and in eq. (33b) we have 
used the Bose and Lorentz symmetries. It is now a simple matter to write the expression (31) in the form

$$
\begin{aligned}
& X_{n}\left(p_{1}, \ldots, p_{n}\right)=\sum_{i=1}^{3} \sum_{\Omega} \prod_{\gamma \in \Omega}\left(-t_{\gamma}^{(0)}\right) \widetilde{\Gamma}_{n}^{\mathrm{u}}\left(\tilde{O}_{i}, p_{1}, \ldots, p_{n}\right) G_{i} \\
& \quad=\sum_{i=1}^{3} \widetilde{\Gamma}_{n}\left(\tilde{O}_{i}, p_{1}, \ldots, p_{n}\right) G_{i},
\end{aligned}
$$

where the local operator vertices $\widetilde{O}_{i}(i=1,2,3)$ are given in eqs. $(10 \mathrm{a}-\mathrm{c})$. Note that oversubtractions for $H_{n}^{\mathrm{u}}$ in eq. (32) turned into minimal subtractions for $\widetilde{\Gamma}_{n}^{\mathrm{u}}\left(\widetilde{O}_{i}, p_{1}\right.$, $\ldots, p_{n}$ ) in eq. (34). Due to the decoupling theorem, the constants $G_{1}, G_{2}, G_{3}$ defined in eqs. $(33 \mathrm{a}-\mathrm{c})$ are all of order $1 / M^{2}$. On the other hand, it is a simple exercise in application of the Weinberg power-counting theorem [9] to prove that the oversubtracted function $\bar{H}_{n}\left(p_{1}, \ldots, p_{n}\right)$ in eq. (32) is at most of order $1 / M^{4}$. Thus, in the limit of $\mu, p_{1}, \ldots, p_{n}<<M$, eq. (32) yields the factorization

$$
\begin{gathered}
H_{n}\left(p_{1}, \ldots, p_{n}\right)=\sum_{i=1}^{3} \widetilde{\Gamma}_{n}\left(\widetilde{O}_{i} p_{1}, \ldots, p_{n}\right) G_{i}+\mathrm{O}\left(\frac{1}{M^{4}}\right) \\
=\frac{1}{M^{2}} \sum_{i=1}^{3} \widetilde{\Gamma}_{n}\left(\widetilde{O}_{i}, p_{1}, \ldots, p_{n}\right) C_{i}+\mathrm{O}\left(\frac{1}{M^{4}}\right)
\end{gathered}
$$

where we have defined $C_{i}=M^{2} G_{i}(i=1,2,3)$.

Once the factorization (9) for proper vertex functions is established, it is a simple matter to deduce the factorization for amputated, connected, Green functions (i.e., matrix elements). Let us denote the amputated, connected, Green function in the $\phi-\psi$ theory with $n$ external $\phi$-legs (of momenta $p_{1}, \ldots, p_{n}$ ) and no external $\psi$-legs by $T_{\mathrm{n}, 0}\left(p_{1}, \ldots, p_{n}\right)$, and the corresponding function with $n$ external $\phi$-legs in the $\phi$ theory by $\widetilde{T}_{n}\left(p_{1}, \ldots, p_{n}\right)$. The functions $T_{n, 0}\left(p_{1}, \ldots, p_{n}\right)$ and $\widetilde{T}_{n}\left(p_{1}, \ldots, p_{n}\right)$ can be always represented as skeleton graphs in respective theories, with all the skeleton blobs corresponding to proper vertex functions and all the bridge lines between these skeleton blobs corresponding to one-particle-reducible lines. One-particlereducible lines are lines with the property that, when any of those lines is cut, the whole graph becomes disconnected. Consequently, the four-momentum assigned to any bridge line is uniquely given as a certain linear combination of external momenta $p_{1}, \ldots, p_{n}$, and thus should be considered as being much smaller than $M$ in the limit we are concerned. Moreover, it is obvious that, with all the external legs for the whole graph corresponding to $\phi$-fields, all the one-particle-reducible lines will be $\phi$-lines in both theories being considered; i.e., skeleton blobs for the function $T_{n, 0}\left(p_{1}, \ldots, p_{n}\right)$ correspond to either $\widetilde{\Gamma}_{m}$ or $H_{m}$. Those sekeleton graphs for $T_{n, 0}\left(p_{1}, \ldots, p_{n}\right)$ with all the skeleton blobs corresponding to $\widetilde{\Gamma}_{m}$ will precisely reproduce $\widetilde{T}_{n}\left(p_{1}, \ldots, p_{n}\right)$. On the other hand, for the skeleton graphs with a single 
$H$-blob and all the rest corresponding to $\widetilde{\Gamma}$-blobs, we may use the factorization (9) for the $H$-blob. This obviously turns the proper vertex functions with $\widetilde{O}_{i}$ once inserted, into the amputated Green functions with $\widetilde{O}_{i}$ once inserted, $\widetilde{T}_{n}\left(\widetilde{O}_{i}, p_{1}, \ldots, p_{n}\right)$. Skeleton graphs with $H$-blobs more than one yield at most $\mathrm{O}\left(1 / M^{4}\right)$ contributions, due to the decoupling theorem [4]. We may thus conclude that amputated, connected, Green functions factorize in the form

$$
T_{n, 0}\left(p_{1}, \ldots, p_{n}\right)=\widetilde{T}_{n}\left(p_{1}, \ldots, p_{n}\right)+\frac{1}{M^{2}} \sum_{i=1}^{3} \widetilde{T}_{n}\left(\tilde{O}_{i}, p_{1}, \ldots, p_{n}\right) C_{i}+\mathrm{O}\left(\frac{1}{M^{4}}\right),
$$

which is the result announced in sect. 1.

\section{The Callan-Symanzik equations}

In sect. 2 we have shown that, up to order $1 / M^{2}$, virtual heavy particles $(\psi)$ induce effective local vertices $\widetilde{O}_{i}(i=1,2,3)$ in the light-particle physics, with the coupling strengths $G_{i} \equiv\left(1 / M^{2}\right) C_{i}$. In renormalized perturbation theory, the constants $C_{i}$ may be directly obtained via eqs. $(33 \mathrm{a}-\mathrm{c})$, or more simply, by matching the both sides of eq. (9), assuming the factorization as stated there. In general, these perturbative calculations yield powers of $\log \left(M^{2} / \mu^{2}\right)$ for the effective coupling strengths $C_{i}$. In this section, we wish to develop a systematic method of summing those powers of logarithms which appear in higher orders. To deal with this problem, the traditional form of the Callan-Symanzik equations [5] needs to be significantly improved due to reasons mentioned in sect. 1. Thus, we may begin with brief discussions on the homogeneous Callan-Symanzik equations developed in ref. [6] ${ }^{*}$.

Consider the following modified $\phi$-field theory (the $\phi^{\prime}$ theory) with the action

$$
\tilde{S}^{\prime}=\int \mathrm{d}^{4} x\left\{\frac{1}{2} \widetilde{\rho}_{\mathrm{B} \phi}\left(\partial_{\mu} \phi\right)^{2}-\frac{1}{2} \tilde{m}_{\mathrm{B}}^{2} \tilde{\rho}_{\mathrm{B} \phi} \phi^{2}-\frac{1}{4 !} \tilde{\alpha}_{\mathrm{B}} \tilde{\rho}_{\mathrm{B} \phi}^{2} \phi^{4}\right\},
$$

which is different from the action (6) only by the presence of $\tilde{m}_{\mathrm{B}}^{2}$ instead of $\tilde{\mu}_{\mathrm{B}}^{2}$. Bare parameters other than $\tilde{m}_{\mathrm{B}}^{2}$ are chosen identically to the ones given in the action (6). As for the bare mass $\tilde{m}_{\mathrm{B}}^{2}$, we write

$$
\tilde{m}_{\mathrm{B}}^{2}=\tilde{\mu}_{\mathrm{B}}^{2}+\tilde{\lambda}_{\mathrm{B}}^{2}=\tilde{\mu}_{\mathrm{B}}^{2}+\lambda^{2} \tilde{Z}_{\mu},
$$

where $\widetilde{Z}_{\mu}$ is independent of $\lambda^{2}$. Thus, as far as (appropriately regulated) bare theories are concerned, the two theories defined by the actions (6) and (35) are identical for $\lambda^{2}=0$. In a subtractive language of defining the full action, we may write

$$
\tilde{S}^{\prime}=\int \mathrm{d}^{4} x\left[\frac{1}{2} \rho_{\phi}\left(\partial_{\mu} \phi\right)^{2}-\frac{1}{2}\left(\mu^{2}+\lambda^{2}\right) \rho_{\phi} \phi^{2}-\frac{1}{4 !} \alpha \rho_{\phi}^{2} \phi^{4}+\tilde{\mathcal{L}}_{\mathrm{c}}^{\prime}\right]
$$

\footnotetext{
* These equations may be viewed as a generalization of the improved renormalization group equation of Weinberg [11].
} 

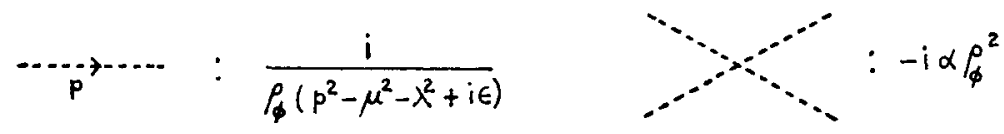

Fig. 5. Feynman rules for the $\phi^{\prime}$ theory.

with

$$
\begin{aligned}
& \mathcal{L}_{\mathrm{c}}^{\prime}=\int \mathrm{d}^{4} x\left\{\frac{1}{2}\left(\tilde{\rho}_{\mathrm{B} \phi}-\rho_{\phi}\right)\left(\partial_{\mu} \phi\right)^{2}-\frac{1}{2}\left[\left(\tilde{\mu}_{\mathrm{B}}^{2}+\tilde{\lambda}_{\mathrm{B}}^{2}\right) \tilde{\rho}_{\mathrm{B} \phi}-\left(\mu^{2}+\lambda^{2}\right) \rho_{\phi}\right] \phi^{2}\right. \\
& \left.\quad-\frac{1}{4}\left[\widetilde{\alpha}_{\mathrm{B}} \tilde{\rho}_{\mathrm{B} \phi}^{2}-\alpha \rho_{\phi}^{2}\right] \phi^{4}\right\} .
\end{aligned}
$$

Note that we introduced $\lambda^{2}$ as a new free parameter of the theory which appears even in the tree approximation. We now must ask whether the bare theory defined by the action (37) yields a consistent renormalized theory. The answer is yes and we will briefly describe the procedure below.

The action (37) with $\widetilde{\mathscr{L}}_{\mathrm{c}}^{\prime}=0$ provides the Feynman rules for the unsubtracted (but appropriately regulated) Green functions. These Feynman rules are shown in fig. 5. Then, the renormalized proper vertex function $\widetilde{\Gamma}_{n}^{\prime}\left(p_{1}, \ldots, p_{n} ; \lambda^{2}\right)$ in this theory may be defined by the forest formula ( $c f$., eq. (14))

$$
\widetilde{\Gamma}_{n}^{\prime}\left(p_{1}, \ldots, p_{n} ; \lambda^{2}\right)=\sum_{\Omega} \prod_{\gamma \in \Omega}\left(-t_{\gamma}^{\prime(0)}\right) \widetilde{\Gamma}_{n}^{\prime \mathrm{u}}\left(p_{1}, \ldots, p_{n} ; \lambda^{2}\right)
$$

where the Taylor operator $t_{\gamma}^{\prime(0)}$ is specified by

$$
\begin{aligned}
& t^{\prime(0)} \widetilde{\Gamma}_{2}^{\prime u}\left(q,-q ; \lambda^{2}\right)=\widetilde{\Gamma}_{2}^{\prime u}\left(q,-q ; \lambda^{2}\right) \underset{q^{2}=\lambda^{2}=0}{\mid}+q^{2}\left[\frac{\partial}{\partial q^{2}} \widetilde{\Gamma}_{2}^{\prime u}\left(q,-q ; \lambda^{2}\right)\right]_{q^{2}=\lambda^{2}=0} \\
& +\lambda^{2}\left[\frac{\partial}{\partial \lambda^{2}} \tilde{\Gamma}_{2}^{\prime u}\left(q,-q, \lambda^{2}\right)\right]_{q^{2}=\lambda^{2}=0}, \\
& t^{\prime(0)} \widetilde{\Gamma}_{4}^{\prime \mathrm{u}}\left(q_{1}, q_{2}, q_{3}, q_{4} ; \lambda^{2}\right)=\left.\widetilde{\Gamma}_{4}^{\prime \mathrm{u}}\left(q_{1}, q_{2}, q_{3}, q_{4} ; \lambda^{2}\right)\right|_{q_{i}=0, \lambda^{2}=0}, \\
& t^{\prime(0)} \widetilde{\Gamma}_{k}^{\prime u}\left(q_{1}, \ldots, q_{k} ; \lambda^{2}\right)=0, \quad(k>4) .
\end{aligned}
$$

It is evident from these subtraction rules that we will have the same bare wave-function parameter and bare coupling constant as the corresponding bare parameters in the action (6), and the total bare mass $\tilde{m}_{\mathrm{B}}^{2}$ will have the form (36). It is also a matter of simple power counting [9] to show that these subtraction rules lead to a finite theory. The renormalized vertex functions in this theory satisfy the following normalization conditions:

$$
\widetilde{\Gamma}_{2}^{\prime}\left(p,-p ; \lambda^{2}\right)_{p^{2}=\lambda^{2}=0}^{\mid}=-i \mu^{2} \rho_{\phi},
$$




$$
\begin{aligned}
& {\left[\frac{\partial}{\partial p^{2}} \widetilde{\Gamma}_{2}^{\prime}\left(p,-p ; \lambda^{2}\right)\right]_{p^{2}=\lambda^{2}=0}=i \rho_{\phi},} \\
& {\left[\frac{\partial}{\partial \lambda^{2}} \tilde{\Gamma}_{2}^{\prime}\left(p,-p ; \lambda^{2}\right)\right]_{p^{2}=\lambda^{2}=0}=-i \rho_{\phi},} \\
& \widetilde{\Gamma}_{4}^{\prime}\left(p_{1}, p_{2}, p_{3}, p_{4} ; \lambda^{2}\right) \underset{p_{i}=0, \lambda^{2}=0}{\mid}=-i \alpha \rho_{\phi}^{2} .
\end{aligned}
$$

Note that, though mass dependence in the unsubtracted functions $\widetilde{\Gamma}_{n}^{\prime u}\left(p_{1}, \ldots, p_{n} ; \lambda^{2}\right)$ always appears in the form of $\mu^{2}+\lambda^{2}$, it is not so with the renormalized functions $\widetilde{\Gamma}_{n}^{\prime}\left(p_{1}, \ldots, p_{n}, \lambda^{2}\right)$. Also, one may note that

$$
\widetilde{\Gamma}_{n}^{\prime}\left(p_{1}, \ldots, p_{n} ; \lambda^{2}=0\right) \equiv \widetilde{\Gamma}_{n}\left(p_{1}, \ldots, p_{n}\right) .
$$

We now derive the Callan-Symanzik equation in the $\phi^{\prime}$ theory. Multiplicative renormalizability of the theory implies that

$$
\widetilde{\Gamma}_{n}^{\prime}\left(p_{1}, \ldots, p_{n} ; \lambda^{2}\right)=\widetilde{\Gamma}_{n}^{\prime \mathrm{u}}\left(p_{1}, \ldots, p_{n} ; \widetilde{m}_{\mathrm{B}}^{2}, \tilde{\rho}_{\mathrm{B} \phi}, \tilde{\alpha}_{\mathrm{B}}, \Lambda^{2}\right),
$$

where $\Lambda^{2}$ is an ultraviolet cutoff and $\widetilde{\Gamma}_{n}^{\prime \prime}\left(p_{1}, \ldots, p_{n} ; \widetilde{m}_{\mathrm{B}}^{2}, \tilde{\rho}_{\mathrm{B} \phi}, \widetilde{\alpha}_{\mathrm{B}}, \Lambda^{2}\right)$ denotes the function obtained by replacing $\mu^{2}+\lambda^{2}, \rho_{\phi}, \alpha$ by $\widetilde{m}_{\mathrm{B}}^{2}, \widetilde{\rho}_{\mathrm{B} \phi}, \widetilde{\alpha}_{\mathrm{B}}$ in the unsubtracted vertex function $\widetilde{\Gamma}_{n}^{\prime \mathbf{u}}\left(p_{1}, \ldots, p_{n} ; \lambda^{2}\right)$. With this relation in mind, we consider the following one-parameter variation:vary $\mu^{2}$ for fixed $\widetilde{m}_{\mathrm{B}}^{2}, \widetilde{\rho}_{\mathrm{B} \phi}, \widetilde{\alpha}_{\mathrm{B}}, \Lambda^{2}$. Under such variation, $\widetilde{\Gamma}_{n}^{\prime \mathbf{u}}\left(p_{1}, \ldots, p_{n} ; \tilde{m}_{\mathrm{B}}^{2}, \tilde{\rho}_{\mathrm{B} \phi}, \widetilde{\alpha}_{\mathrm{B}}, \Lambda^{2}\right)$ is invariant and thus, from eq. (43), we obtain

$$
\begin{aligned}
& \mu^{2} \frac{\widetilde{D}}{D \mu^{2}} \Gamma_{\mathrm{n}}^{\prime} \equiv\left[\mu^{2} \frac{\partial}{\partial \mu^{2}}\right. \\
& \left.\quad+\mu^{2} \frac{\widetilde{D} \alpha}{D \mu^{2}} \frac{\partial}{\partial \alpha}+\mu^{2} \frac{\widetilde{D} \lambda^{2}}{D \mu^{2}} \frac{\partial}{\partial \lambda^{2}}+\mu^{2} \frac{\widetilde{D} \rho_{\phi}}{D \mu^{2}} \frac{\partial}{\partial \rho_{\phi}}\right] \widetilde{\Gamma}_{n}^{\prime}\left(p_{1}, \ldots, p_{n} ; \lambda^{2}\right)=0
\end{aligned}
$$

where $\mu^{2} \widetilde{D} / D \mu^{2}$ denotes the above one-parameter variation.

Here, we may factor out the complete $\rho_{\phi}$ dependence from renormalized vertex functions by writing

$$
\begin{aligned}
& \tilde{\Gamma}_{n}^{\prime}\left(p_{1}, \ldots, p_{n} ; \lambda^{2}\right)=\rho_{\phi}^{n / 2} \widetilde{V}_{n}\left(p_{1}, \ldots, p_{n} ; \lambda^{2}\right), \\
& \widetilde{V}_{n}\left(p_{1}, \ldots, p_{n} ; \lambda^{2}\right)=\widetilde{\Gamma}_{n}^{\prime}\left(p_{1}, \ldots, p_{n} ; \lambda^{2}\right) \underset{\rho_{\phi}=1}{\mid},
\end{aligned}
$$

and also define the dimensionless constants $\widetilde{B}_{\alpha}, \tilde{\gamma}_{\phi}$ by

$$
\widetilde{B}_{\alpha}=\frac{1}{\alpha} \mu^{2} \frac{\tilde{D} \alpha}{D \mu^{2}}, \quad \tilde{\gamma}_{\phi}=\frac{1}{\rho_{\phi}} \mu^{2} \frac{\widetilde{D} \rho_{\phi}}{D \mu^{2}} .
$$


Then, for the function $\widetilde{V}_{n}$, eq. (44) yields the equation

$$
\left[\mu^{2} \frac{\partial}{\partial \mu^{2}}+\widetilde{B}_{\alpha} \alpha \frac{\partial}{\partial \alpha}+\mu^{2} \frac{\widetilde{D} \lambda^{2}}{D \mu^{2}} \frac{\partial}{\partial \lambda^{2}}+\frac{1}{2} n \widetilde{\gamma}_{\phi}\right] \widetilde{V}_{n}\left(p_{1}, \ldots, p_{n} ; \lambda^{2}\right)=0
$$

For the term $\mu^{2} \widetilde{D} \lambda^{2} / D \mu^{2}$ we note from the relation (36) that

$$
0=\mu^{2} \frac{\widetilde{D}}{D \mu^{2}} \tilde{m}_{\mathrm{B}}^{2}=\mu^{2} \frac{\widetilde{D}}{D \mu^{2}} \tilde{\mu}_{\mathrm{B}}^{2}+\lambda^{2} \mu^{2} \frac{\widetilde{D}}{D \mu^{2}} \widetilde{Z}_{\mu}+\widetilde{Z}_{\mu} \mu^{2} \frac{\widetilde{D}}{D \mu^{2}} \lambda^{2} .
$$

From eq. (48), we may thus write

$$
\mu^{2} \frac{\widetilde{D} \lambda^{2}}{D \mu^{2}}=-\mu^{2}\left(1-\widetilde{\Theta}_{1}\right)+\lambda^{2} \widetilde{\Theta}_{2}
$$

where $\widetilde{\Theta}_{1}, \widetilde{\Theta}_{2}$ are dimensionless constants defined by

$$
\widetilde{Z}_{\mu}^{-1} \frac{\widetilde{D}}{D \mu^{2}} \tilde{\mu}_{\mathrm{B}}^{2}=1-\widetilde{\Theta}_{1}, \quad-\mu^{2} \widetilde{Z}_{\mu}^{-1} \frac{\widetilde{D}}{D \mu^{2}} \widetilde{Z}_{\mu}=\Theta_{2} .
$$

Inserting eq. (49) into eq. (47), we obtain the Callan-Symanzik equation of the form [6]

$$
\left\{\mu^{2} \frac{\partial}{\partial \mu^{2}}+\widetilde{B}_{\alpha} \alpha \frac{\partial}{\partial \alpha}-\left[\left(1-\widetilde{\Theta}_{1}\right) \mu^{2}-\widetilde{\Theta}_{2} \lambda^{2}\right] \frac{\partial}{\partial \lambda^{2}}+\frac{1}{2} n \widetilde{\gamma}_{\phi}\right\} \widetilde{V}_{n}\left(p_{1}, \ldots, p_{n} ; \lambda^{2}\right)=0
$$

Since eq. (51) is an equation for renormalized vertex functions, the Callan-Symanzik coefficients $\widetilde{B}_{\alpha}, \widetilde{\Theta}_{1}, \widetilde{\Theta}_{2}, \widetilde{\gamma}_{\phi}$ should be finite and, according to our definitions, muct be dimensionless functions of $\alpha$ only without any dependence on $\lambda^{2} / \mu^{2}$. In fact, these coefficients can be expressed in terms of appropriate derivatives of renormalized vertex functions, viz.,

$$
\begin{aligned}
& \widetilde{\Theta}_{1}=-\tilde{\gamma}_{\phi} \\
& i \widetilde{\gamma}_{\phi}-\left(1-\widetilde{\Theta}_{1}\right) \mu^{2}\left[\frac{\partial}{\partial \lambda^{2}} \frac{\partial}{\partial p^{2}} \widetilde{V}_{2}\left(p,-p ; \lambda^{2}\right)\right]_{p^{2}=\lambda^{2}=0}=0 \\
& -i\left(\widetilde{\Theta}_{2}+\tilde{\gamma}_{\phi}\right)-\left(1-\widetilde{\Theta}_{1}\right) \mu^{2}\left[\frac{\partial^{2}}{\partial\left(\lambda^{2}\right)^{2}} \widetilde{V}_{2}\left(p,-p ; \lambda^{2}\right)\right]_{p^{2}=\lambda^{2}=0}=0 \\
& -i \widetilde{B}_{\alpha} \alpha-2 i \tilde{\gamma}_{\phi} \alpha-\left(1-\widetilde{\Theta}_{1}\right) \mu^{2}\left[\frac{\partial}{\partial \lambda^{2}} \tilde{V}_{4}\left(p_{1}, p_{2}, p_{3}, p_{4} ; \lambda^{2}\right)\right]_{p_{i}=0, \lambda^{2}=0}=0
\end{aligned}
$$

Eqs. (52a-d) determine the Callan-Symanzik coefficients $\widetilde{B}_{\alpha}, \widetilde{\Theta}_{1}, \widetilde{\Theta}_{2}, \widetilde{\gamma}_{\phi}$ completely. Here, it may be worthwhile to note that, upon setting $\lambda^{2}=0$, eq. (51) reduces to the conventional Callan-Symanzik equation [5] with its right-hand side written 
in the form,

$$
\left(1-\widetilde{\Theta}_{1}\right) \mu^{2}\left[\frac{\partial}{\partial \lambda^{2}} \widetilde{V}_{n}\left(p_{1}, \ldots, p_{n} ; \lambda^{2}\right)\right]_{\lambda^{2}=0} .
$$

We now turn to the $\phi-\psi$ theory. To obtain the Callan-Symanzik equation which is useful for our purpose, we consider the following field theory, called the $(\phi-\psi)^{\prime}$ theory, with the action

$$
\begin{aligned}
S^{\prime} & =\int \mathrm{d}^{4} x\left\{\frac{1}{2} \rho_{\mathrm{B} \phi}\left(\partial_{\mu} \phi\right)^{2}+\frac{1}{2} \rho_{\mathrm{B} \psi}\left(\partial_{\mu} \psi\right)^{2}-\frac{1}{2} m_{\mathrm{B}}^{2} \rho_{\mathrm{B} \phi} \phi^{2}-\frac{1}{2} M_{\mathrm{B}}^{\prime 2} \rho_{\mathrm{B} \psi} \psi^{2}\right. \\
& \left.-\frac{1}{4 !} \alpha_{\mathrm{B}} \rho_{\mathrm{B} \phi}^{2} \phi^{4}-\frac{1}{4 !} \beta_{\mathrm{B}} \rho_{\mathrm{B} \psi}^{2} \psi^{4}-\frac{1}{4} \gamma_{\mathrm{B}} \rho_{\mathrm{B} \psi} \rho_{\mathrm{B} \phi} \phi^{2} \psi^{2}\right\} \\
& =\int \mathrm{d}^{4} x\left\{\frac{1}{2} \rho_{\phi}\left(\partial_{\mu} \phi\right)^{2}+\frac{1}{2} \rho_{\psi}\left(\partial_{\mu} \psi\right)^{2}-\frac{1}{2}\left(\mu^{2}+\lambda^{2}\right) \rho_{\phi} \phi^{2}-\frac{1}{2}\left(M^{2}+\kappa^{2}\right) \rho_{\psi} \psi^{2}\right. \\
& \left.-\frac{1}{4 !} \alpha \rho_{\phi}^{2} \phi^{4}-\frac{1}{4 !} \beta \rho_{\phi}^{2} \psi^{4}-\frac{1}{4} \gamma \rho_{\psi} \rho_{\phi} \phi^{2} \psi^{2}+\mathcal{L}_{\mathrm{c}}^{\prime}\right\}
\end{aligned}
$$

where the bare parameters $\rho_{\mathrm{B} \phi}, \rho_{\mathrm{B} \psi}, \alpha_{\mathrm{B}}, \beta_{\mathrm{B}}, \gamma_{\mathrm{B}}$ are identical to the corresponding bare parameters used in the action (3) and the bare masses $m_{\mathrm{B}}^{2}, M_{\mathrm{B}}^{\prime 2}$ are given by

$$
\begin{aligned}
& m_{\mathrm{B}}^{2}=\mu_{\mathrm{B}}^{2}+\lambda^{2} Z_{\mu}+\kappa^{2} Z_{\mu}^{\prime}, \\
& M_{\mathrm{B}}^{\prime 2}=M_{\mathrm{B}}^{2}+\lambda^{2} Z_{M}+\kappa^{2} Z_{M}^{\prime} .
\end{aligned}
$$

In eq. (55), $\mu_{\mathrm{B}}^{2}$ and $M_{\mathrm{B}}^{2}$ are the same bare masses used in the action (3), and $Z_{\mu}, Z_{\mu}^{\prime}$, $Z_{M}, Z_{M}^{\prime}$ are independent of $\lambda^{2}, \kappa^{2}$. The corresponding renormalized theory can be defined as below.

The action (54) with $\mathcal{L}_{\mathrm{c}}^{\prime} \equiv 0$ provides the Feynman rules for unsubtracted Green functions. These Feynman rules are shown in fig. 6 . Then, the renormalized proper vertex function $\Gamma_{n, N}^{\prime}\left(p_{1}, \ldots, p_{n}, P_{1}, \ldots, P_{N} ; \lambda^{2}, \kappa^{2}\right)$ may be given by the forest formula [7]

$$
\begin{aligned}
& \Gamma_{n, N}^{\prime}\left(p_{1}, \ldots, p_{n}, P_{1}, \ldots, P_{N} ; \lambda^{2}, \kappa^{2}\right) \\
& \quad=\sum_{\Omega} \sum_{\gamma \in \Omega}\left(-t_{\gamma}^{\prime(0)}\right) \Gamma_{n, N}^{\prime \mathrm{u}}\left(p_{1}, \ldots, p_{n}, P_{1}, \ldots, P_{N} ; \lambda^{2}, \kappa^{2}\right),
\end{aligned}
$$

where the Taylor operator $t_{\gamma}^{\prime(0)}$ is the minimum subtraction operator analogous to the one specified by eqs. $(40 \mathrm{a}-\mathrm{c})$, this time treating external momenta, $\lambda^{2}$, and $\kappa^{2}$ on an equal footing. The vertex functions renormalized in such a manner will satisfy the following normalization conditions:

$$
\Gamma_{2,0}^{\prime}\left(p,-p ; \lambda^{2}, \kappa^{2}\right) \underset{p^{2}=\lambda^{2}=\kappa^{2}=0}{\mid}=-i \mu^{2} \rho_{\phi},
$$




$$
\begin{aligned}
& \frac{\partial}{\partial p^{2}} \Gamma_{2,0}^{\prime}\left(p,-p ; \lambda^{2} \kappa^{2}\right) \underset{p^{2}=\lambda^{2}=\kappa^{2}=0}{\mid}=i \rho_{\phi}, \\
& \frac{\partial}{\partial \lambda^{2}} \Gamma_{2,0}^{\prime}\left(p,-p ; \lambda^{2}, \kappa^{2}\right) \underset{p^{2}=\lambda^{2}=\kappa^{2}=0}{\left.\right|^{2}}=-i \rho_{\phi}, \\
& \frac{\partial}{\partial \kappa^{2}} \Gamma_{2,0}^{\prime}\left(p,-p ; \lambda^{2}, \kappa^{2}\right) \underset{p^{2}=\lambda^{2}=\kappa^{2}=0}{\mid}=0, \\
& \left.\Gamma_{4,0}^{\prime}\left(p_{1}, p_{2}, p_{3}, p_{4} ; \lambda^{2}, \kappa^{2}\right)\right|_{p_{i}=0, \lambda^{2}=\kappa^{2}=0}=-i \alpha \rho_{\phi}^{2} \text {, } \\
& \Gamma_{0,2}^{\prime}\left(P,-P ; \lambda^{2}, \kappa^{2}\right) \underset{P^{2}=M^{2}, \lambda^{2}=\kappa^{2}=0}{\mid}=0, \\
& \frac{\partial}{\partial P^{2}} \Gamma_{0,2}^{\prime}\left(P,-P ; \lambda^{2}, \kappa^{2}\right) \underset{P^{2}=M^{2}, \lambda^{2}=\kappa^{2}=0}{\mid}=i \rho_{\psi} \text {, } \\
& \frac{\partial}{\partial \lambda^{2}} \Gamma_{0,2}^{\prime}\left(P,-P ; \lambda^{2}, \kappa^{2}\right) \underset{P^{2}=M^{2}, \lambda^{2}=\kappa^{2}=0}{\left.\right|_{\Omega^{2}}}=0 \text {, } \\
& \frac{\partial}{\partial \kappa^{2}} \Gamma_{0,2}^{\prime}\left(P,-P ; \lambda^{2}, \kappa^{2}\right) \underset{P^{2}=M^{2}, \lambda^{2}=\kappa^{2}=0}{\left.\right|^{2}}=-i \rho_{\psi}, \\
& \Gamma_{0,4}^{\prime}\left(P_{1}, P_{2}, P_{3}, P_{4} ; \lambda^{2} \kappa^{2}\right) \\
& P_{i}^{2}=M^{2}, \lambda^{2}=\kappa^{2}=0 \\
& \begin{array}{c}
P_{i}^{2}=M^{2}, \lambda^{2}=\kappa=0 \\
\left(P_{1}+P_{2}\right)^{2}=\left(P_{2}+P_{3}\right)^{2}=\left(P_{1}+P_{3}\right)^{2}={ }_{3}^{4} M^{2}
\end{array} \\
& =-i \beta \rho_{\psi}^{2} \\
& \Gamma_{2,2}^{\prime}\left(p_{1}, p_{2}, P_{1}, P_{2} ; \lambda^{2}, \kappa^{2}\right){ }_{p_{i}=0, \lambda^{2}=\kappa^{2}=0, P_{1}^{2}=P_{2}^{2}=M^{2}}^{\mid}=-i \gamma \rho_{\phi} \rho_{\psi} .
\end{aligned}
$$

One should here note that, although all the mass dependences in the unsubtracted functions $\Gamma_{n, N}^{\prime u}\left(p_{1}, \ldots, p_{n}, P_{1}, \ldots, P_{N} ; \lambda^{2}, \kappa^{2}\right)$ appear only in the form of $\mu^{2}+\lambda^{2}$ and $M^{2}+\kappa^{2}$, the above renormalization procedure will not preserve such combined mass dependences for the renormalized functions $\Gamma_{n, N}^{\prime}\left(p_{1}, \ldots p_{n}, P_{1}, \ldots, P_{N}\right.$; $\left.\lambda^{2}, k^{2}\right)$. It is also clear that

$$
\Gamma_{n, N}^{\prime}\left(p_{1}, \ldots, p_{n}, P_{1}, \ldots, P_{N} ; \lambda^{2}=\kappa^{2}=0\right) \equiv \Gamma_{n, N}\left(p_{1}, \ldots, p_{n}, P_{1}, \ldots, P_{N}\right) .
$$

To derive the Callan-Symanzik equation in the $(\phi-\psi)^{\prime}$ theory, we first note that multiplicative renormalizability of the theory implies the relation

$$
\begin{aligned}
& \Gamma_{n, N}^{\prime}\left(p_{1}, \ldots, p_{n}, P_{1}, \ldots, P_{N} ; \lambda^{2}, \kappa^{2}\right)=\Gamma_{n, N}^{\prime u}\left(p_{1}, \ldots, p_{n}, P_{1}, \ldots, P_{N} ;\right. \\
& \left.\quad m_{\mathrm{B}}^{2}, M_{\mathrm{B}}^{\prime 2}, \rho_{\mathrm{B} \phi}, \rho_{\mathrm{B} \psi}, \alpha_{\mathrm{B}}, \beta_{\mathrm{B}}, \gamma_{\mathrm{B}}, \Lambda^{2}\right),
\end{aligned}
$$



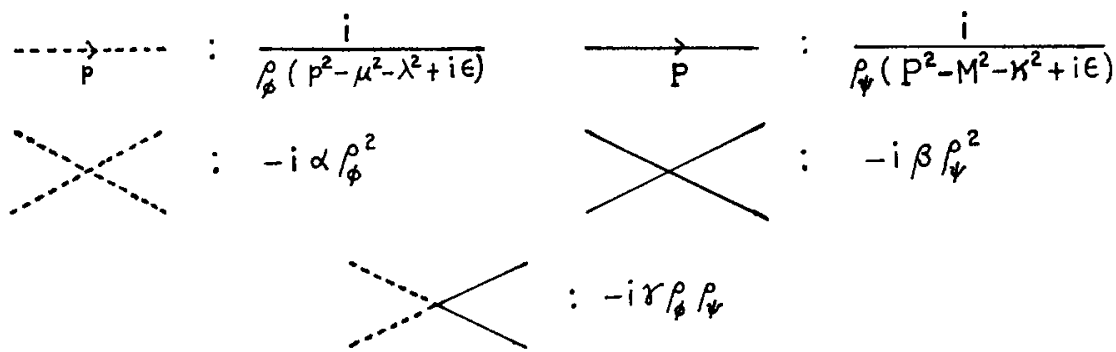

Fig. 6. Feynman rules for the $(\phi-\psi)^{\prime}$ theory.

where

$$
\Gamma_{n, N}^{\prime \mathrm{u}}\left(p_{1}, \ldots, p_{n}, P_{1}, \ldots, P_{N} ; m_{\mathrm{B}}^{2}, M_{\mathrm{B}}^{\prime 2}, \rho_{\mathrm{B} \phi}, \rho_{\mathrm{B} \psi}, \alpha_{\mathrm{B}}, \beta_{\mathrm{B}}, \gamma_{\mathrm{B}}, \Lambda^{2}\right)
$$

represents the function obtained from the unsubtracted function $\Gamma_{n, N}^{\prime u}\left(p_{1}, \ldots, p_{n}\right.$, $\left.P_{1}, \ldots, P_{N} ; \lambda^{2}, \kappa^{2}\right)$ by replacing $\mu^{2}+\lambda^{2}, M^{2}+\kappa^{2}, \rho_{\phi}, \rho_{\psi}, \alpha, \beta, \gamma$ with $m_{\mathrm{B}}^{2}, M_{\mathrm{B}}^{\prime 2}$, $\rho_{\mathbf{B} \phi}, \rho_{\mathbf{B} \psi}, \alpha_{\mathrm{B}}, \beta_{\mathbf{B}}, \gamma_{\mathrm{B}}$. We are here interested in obtaining an equation for $\Gamma_{n, 0}^{\prime}\left(p_{1}, \ldots, p_{\mathrm{n}}, \lambda^{2}, \kappa^{2}\right)$ which will be useful in studying the limit $\mu, \lambda, \kappa, p_{1}, \ldots, p_{n}$ $<<M$. Such an equation can be obtained by considering the variation of $\mu^{2}$ for fixed $m_{\mathrm{B}}^{2}, M^{2}, M_{\mathrm{B}}^{\prime 2}, \rho_{\mathrm{B} \phi}, \rho_{\mathrm{B} \psi}, \alpha_{\mathrm{B}}, \beta_{\mathrm{B}}, \gamma_{\mathrm{B}}, \Lambda^{2}$. Since $\Gamma_{n, N}^{\prime \mathrm{u}}\left(p_{1}, \ldots, p_{n}, P_{1}, \ldots, P_{N}\right.$; $m_{\mathrm{B}}^{2}, M_{\mathrm{B}}^{\prime 2}, \rho_{\mathrm{B} \phi} \rho_{\mathrm{B} \psi}, \alpha_{\mathrm{B}}, \beta_{\mathrm{B}}, \gamma_{\mathrm{B}}, \Lambda^{2}$ ) is invariant under that variation, eq. (59) yields the following Callan-Symanzik equation for the renormalized proper vertex function:

$$
\begin{aligned}
& \mu^{2} \frac{D}{D \mu^{2}} \Gamma_{n, N}^{\prime} \equiv\left[\mu^{2} \frac{\partial}{\partial \mu^{2}}+\mu^{2} \frac{D \alpha}{D \mu^{2}} \frac{\partial}{\partial \alpha}+\mu^{2} \frac{D \beta}{D \mu^{2}} \frac{\partial}{\partial \beta}+\mu^{2} \frac{D \gamma}{D \mu^{2}} \frac{\partial}{\partial \gamma}+\mu^{2} \frac{D \lambda^{2}}{D \mu^{2}} \frac{\partial}{\partial \lambda^{2}}\right. \\
& \left.\quad+\mu^{2} \frac{D \kappa^{2}}{D \mu^{2}} \frac{\partial}{\partial \kappa^{2}}+\mu^{2} \frac{D \rho_{\phi}}{D \mu^{2}} \frac{\partial}{\partial \rho_{\phi}}+\mu^{2} \frac{D \rho_{\psi}}{D \mu^{2}}\right] \Gamma_{n, N}^{\prime}\left(p_{1}, \ldots, p_{n}, P_{1}, \ldots, P_{N} ; \lambda^{2}, \kappa^{2}\right) \\
& \quad=0 .
\end{aligned}
$$

As in the case of the $\phi^{\prime}$ theory, we may factor out $\rho_{\phi}$ and $\rho_{\psi}$ dependences by writing

$$
\begin{aligned}
& \Gamma_{n, N}^{\prime}\left(p_{1}, \ldots, p_{n}, P_{1}, \ldots, P_{N} ; \lambda^{2}, \kappa^{2}\right)=\rho_{\phi}^{n / 2} \rho_{\psi}^{n / 2} V_{n, N}\left(p_{1}, \ldots, p_{n}, P_{1}, \ldots, P_{N} ; \lambda^{2}, \kappa^{2}\right), \\
& V_{n, N}\left(p_{1}, \ldots, p_{n}, P_{1}, \ldots, P_{N} ; \lambda^{2}, \kappa^{2}\right)=\Gamma_{n, N}^{\prime}\left(p_{1}, \ldots, p_{n}, P_{1}, \ldots, P_{N} ; \lambda^{2}, \kappa^{2}\right) \underset{\rho_{\phi}=\rho_{\psi}=1}{\mid},
\end{aligned}
$$

and define the Callan-Symanzik coefficients $B_{\alpha}, B_{\beta}, B_{\gamma}, \gamma_{\phi}, \gamma_{\psi}$ by

$$
B_{\alpha}=\frac{1}{\alpha} \mu^{2} \frac{D \alpha}{D \mu^{2}}, \quad B_{\beta}=\frac{1}{\beta} \mu^{2} \frac{D \beta}{D \mu^{2}}, \quad B_{\gamma}=\frac{1}{\gamma} \mu^{2} \frac{D \gamma}{D \mu^{2}},
$$




$$
\gamma_{\phi}=\frac{1}{\rho_{\phi}} \mu^{2} \frac{D \rho_{\phi}}{D \mu^{2}}, \quad \gamma_{\psi}=\frac{1}{\rho_{\psi}} \mu^{2} \frac{D \rho_{\psi}}{D \mu^{2}} .
$$

For $\mu^{2} D \lambda^{2} / D \mu^{2}$ and $\mu^{2} D \kappa^{2} / D \mu^{2}$, we note from eq. (55) that

$$
\begin{aligned}
& \mu^{2} \frac{D \lambda^{2}}{D \mu^{2}}=\frac{-1}{\left|\begin{array}{ll}
Z_{\mu} & Z_{\mu}^{\prime} \\
Z_{M} & Z_{M}^{\prime}
\end{array}\right|}\left|\begin{array}{ll}
\mu^{2} \frac{D \mu_{\mathrm{B}}^{2}}{D \mu^{2}}+\lambda^{2} \mu^{2} \frac{D Z_{\mu}}{D \mu^{2}}+\kappa^{2} \mu^{2} \frac{D Z_{\mu}^{\prime}}{D \mu^{2}} & Z_{\mu}^{\prime} \\
\mu^{2} \frac{D M_{\mathrm{B}}^{2}}{D \mu^{2}}+\lambda^{2} \mu^{2} \frac{D Z_{M}}{D \mu^{2}}+\kappa^{2} \mu^{2} \frac{D Z_{M}^{\prime}}{D \mu^{2}} & Z_{M}^{\prime}
\end{array}\right| \\
& \equiv-\mu^{2}\left(1-\Theta_{1}\right)+\lambda^{2} \Theta_{2}+\kappa^{2} \Theta_{3}, \\
& \mu^{2} \frac{D \kappa^{2}}{D \mu^{2}}=\frac{-1}{\left|\begin{array}{ll}
Z_{\mu} & Z_{\mu}^{\prime} \\
Z_{M} & Z_{M}^{\prime}
\end{array}\right|}\left|\begin{array}{ll}
Z_{\mu} & \mu^{2} \frac{D \mu_{\mathrm{B}}^{2}}{D \mu^{2}}+\lambda^{2} \mu^{2} \frac{D Z_{\mu}}{D \mu^{2}}+\kappa^{2} \mu^{2} \frac{D Z_{\mu}^{\prime}}{D \mu^{2}} \\
Z_{M} & \mu^{2} \frac{D M_{\mathrm{B}}^{2}}{D \mu^{2}}+\lambda^{2} \mu^{2} \frac{D Z_{M}}{D \mu^{2}}+\kappa^{2} \mu^{2} \frac{D Z_{M}^{\prime}}{D \mu^{2}}
\end{array}\right| \\
& \equiv \mu^{2} \Omega_{1}+\lambda^{2} \Omega_{2}+\kappa^{2} \Omega_{3},
\end{aligned}
$$

where we have defined $\lambda^{2}$ and $\kappa^{2}$ independent constants $\Theta_{1}, \Theta_{2}, \Theta_{3}, \Omega_{1}, \Omega_{2}, \Omega_{3}$. Inserting eqs. (62)-(64) into eq. (60), we obtain the Callan-Symanzik equation of the form

$$
\begin{aligned}
& \left\{\mu^{2} \frac{\partial}{\partial \mu^{2}}+B_{\alpha} \alpha \frac{\partial}{\partial \alpha}+B_{\beta} \beta \frac{\partial}{\partial \beta}+B_{\gamma} \gamma \frac{\partial}{\partial \gamma}-\left[\mu^{2}\left(1-\Theta_{1}\right)-\lambda^{2} \Theta_{2}-\kappa^{2} \Theta_{3}\right] \frac{\partial}{\partial \lambda^{2}}\right. \\
& \left.+\left[\mu^{2} \Omega_{1}+\lambda^{2} \Omega_{2}+\kappa^{2} \Omega_{3}\right] \frac{\partial}{\partial \kappa^{2}}+\frac{n}{2} \gamma_{\phi}+\frac{N}{2} \gamma_{\psi}\right\} V_{n, N}\left(p_{1}, \ldots, p_{n}, P_{1}, \ldots, P_{N}\right. \\
& \left.\lambda^{2}, \kappa^{2}\right)=0 .
\end{aligned}
$$

Since eq. (65) is an equation for renormalized vertex functions, the Callan-Symanzik coefficients $B_{\alpha}, B_{\beta}, B_{\gamma}, \Theta_{1}, \Theta_{2}, \Theta_{3}, \Omega_{1}, \Omega_{2}, \Omega_{3}, \gamma_{\phi}, \gamma_{\psi}$ should be finite and moreover, according to the definitions given in eqs. (62) $-(64)$, dimensionless functions of $\alpha, \beta, \gamma, \mu^{2} / M^{2}$. As was previously done in the $\phi^{\prime}$ theory, we may again express these Callan-Symanzik coefficients explicitly in terms of appropriate derivatives of renormalized vertex functions as follows:

$$
\begin{aligned}
& \Theta_{1}=-\gamma_{\phi}, \\
& \Omega_{1}=0 \\
& i \gamma_{\phi}-\left(1-\Theta_{1}\right) \mu^{2}\left[\frac{\partial}{\partial \lambda^{2}} \frac{\partial}{\partial p^{2}} V_{2,0}\left(p,-p ; \lambda^{2}, \kappa^{2}\right)\right]_{p^{2}=\lambda^{2}=\kappa^{2}=0}=0,
\end{aligned}
$$




$$
\begin{aligned}
& -i\left(\Theta_{2}+\gamma_{\phi}\right)-\left(1-\Theta_{1}\right) \mu^{2}\left[\frac{\partial^{2}}{\partial\left(\lambda^{2}\right)^{2}} V_{2,0}\left(p,-p ; \lambda^{2}, \kappa^{2}\right)\right]_{p^{2}=\lambda^{2}=\kappa^{2}=0}=0 \\
& -i \Theta_{3}-\left(1-\Theta_{1}\right) \mu^{2}\left[\frac{\partial}{\partial \lambda^{2}} \frac{\partial}{\partial \kappa^{2}} V_{2,0}\left(p,-p ; \lambda^{2}, \kappa^{2}\right)\right]_{p^{2}=\lambda^{2}=\kappa^{2}=0}=0, \quad(66 \mathrm{e}) \\
& i \gamma_{\psi}-\left(1-\Theta_{1}\right) \mu^{2}\left[\frac{\partial}{\partial \lambda^{2}} \frac{\partial}{\partial P^{2}} V_{0,2}\left(P,-P ; \lambda^{2}, \kappa^{2}\right)\right]_{P^{2}=M^{2}, \lambda^{2}=\kappa^{2}=0}=0, \quad(66 \mathrm{f}) \\
& -i \Omega_{2}-\left(1-\Theta_{1}\right) \mu^{2}\left[\frac{\partial^{2}}{\partial\left(\lambda^{2}\right)^{2}} V_{0,2}\left(P,-P ; \lambda^{2}, \kappa^{2}\right)\right]_{P^{2}=M^{2}, \lambda^{2}=\kappa^{2}=0}=0, \quad(66 \mathrm{~g}) \\
& -i\left(\Omega_{3}+\gamma_{\psi}\right)-\left(1-\Theta_{1}\right) \mu^{2}\left[\frac{\partial}{\partial \lambda^{2}} \frac{\partial}{\partial \kappa^{2}} V_{0,2}\left(P,-P ; \lambda^{2}, \kappa^{2}\right)\right]_{P^{2}=M^{2}, \lambda^{2}=\kappa^{2}=0}=0
\end{aligned}
$$

$$
-i B_{\alpha} \alpha-2 i \gamma \alpha-\left(1-\Theta_{1}\right) \mu^{2}\left[\frac{\partial}{\partial \lambda^{2}} V_{4,0}\left(p_{1}, \ldots, p_{4} ; \lambda^{2}, \kappa^{2}\right)\right]_{p_{i}=0, \lambda^{2}=\kappa^{2}=0}=0
$$

$$
\begin{aligned}
& -i B_{\beta} \beta-2 i \gamma_{\psi} \beta-\left(1-\Theta_{1}\right) \mu^{2}\left[\frac{\partial}{\partial \lambda^{2}}\right. \\
& \left.\times V_{0,4}\left(P_{1}, \ldots, P_{4} ; \lambda^{2}, \kappa^{2}\right)\right]\left.{ }_{\left(P_{1}+P_{2}\right)^{2}=\left(P_{2}+P_{3}\right)^{2}=\left(P_{1}+P_{3}\right)^{2}=\frac{4}{3} M^{2}}^{\lambda^{2}=\kappa^{2}=0, P_{i}^{2}=M^{2}}\right|_{-i B_{\gamma} \gamma-i \gamma_{\phi} \gamma-i \gamma_{\psi} \gamma-\left(1-\Theta_{1}\right) \mu^{2}\left[\frac{\partial}{\partial \lambda^{2}} V_{2,2}\right.}=0 . \\
& \left.\quad \times\left(p_{1}, p_{2}, P_{1}, P_{2} ; \lambda^{2}, \kappa^{2}\right)\right]_{p_{i}=0, \lambda^{2}=\kappa^{2}=0, P_{1}^{2}=P_{2}^{2}=M^{2}}^{\mid}
\end{aligned}
$$


of the primed theories. We now want to study what kind of information these Callan-Symanzik equations provide us for the function $\Gamma_{n, 0}\left(p_{1}, \ldots, p_{n}\right)$ in the limit of $\mu, p_{1}, \ldots, p_{n}<<M$, or more generally, for the function $\Gamma_{n, 0}^{\prime}\left(p_{1}, \ldots, p_{n} ; \lambda^{2}, \kappa^{2}\right)$ in the limit of $\mu, \lambda, \kappa, p_{1}, \ldots, p_{n}<<M$. As will become evident later, consideration of $\Gamma_{n, 0}^{\prime}\left(p_{1}, \ldots, p_{n}, \lambda^{2}, \kappa^{2}\right)$ here is an important key to our analysis, since this will allow us to handle the changes in the Callan-Symanzik coefficients systemmatically. (See eqs. $(52 \mathrm{a}-\mathrm{d})$ and $(66 \mathrm{a}-\mathrm{k})$, which express various Callan-Symanzik coefficients in terms of appropriate derivatives of renormalized vertex functions.)

As a prerequisite for such analysis, we have to first establish the factorization analogous to eq. (9), for the function $\Gamma_{n, 0}^{\prime}\left(p_{1}, \ldots, p_{n}, \lambda^{2}, \kappa^{2}\right)$ in the limit of $\mu, \lambda, \kappa, p_{1}, \ldots, p_{n}<<M$. Since its derivation is almost parallel to the one given for $\Gamma_{n, 0}\left(p_{1}, \ldots, p_{n}\right)$ in sect. 2 , here we will only indicate main steps leading to the final result. Write the unsubtracted function

$$
H_{n}^{\prime \mathrm{u}}\left(p_{1}, \ldots, p_{n}, \lambda^{2}, \kappa^{2}\right)=\Gamma_{n, 0}^{\prime \mathrm{u}}\left(p_{1}, \ldots, p_{n} ; \lambda^{2}, \kappa^{2}\right)-\tilde{\Gamma}_{n}^{\prime u}\left(p_{1}, \ldots, p_{n} ; \lambda^{2}\right),
$$

and the corresponding renormalized function

$$
\begin{aligned}
& H_{n}^{\prime}\left(p_{1}, \ldots, p_{n} ; \lambda^{2}, \kappa^{2}\right) \equiv \Gamma_{n, 0}^{\prime}\left(p_{1}, \ldots, p_{n}, \lambda^{2}, \kappa^{2}\right)-\tilde{\Gamma}_{n}^{\prime}\left(p_{1}, \ldots, p_{n} ; \lambda^{2}\right) \\
& \quad=\sum_{\Omega} \prod_{\gamma \in \Omega}\left(-t_{\gamma}^{\prime(0)}\right) H_{n}^{\prime u}\left(p_{1}, \ldots, \mathrm{p}_{n} ; \lambda^{2}, \kappa^{2}\right) .
\end{aligned}
$$

We also define the oversubtracted function

$$
\bar{H}_{n}^{\prime}\left(p_{1}, \ldots, p_{n} ; \lambda^{2}, \kappa^{2}\right)=\sum_{\Omega} \prod_{\gamma \in \Omega}\left(-\bar{t}_{\gamma}^{\prime(0)}\right) H_{n}^{\prime u}\left(p_{1}, \ldots, p_{n} ; \lambda^{2}, \kappa^{2}\right)
$$

with the Taylor operator $\bar{t}_{\gamma}^{\prime}$ siven by

$$
\begin{array}{ll}
\bar{t}_{\gamma}^{\prime}=t_{\gamma}^{\prime(2)}, & \begin{array}{l}
\text { if } \gamma \text { corresponds to } H_{2}^{\prime \mathrm{u}}, H_{4}^{\prime \mathrm{u}}, \text { or } H_{6}^{\prime \mathrm{u}} \text { and there are } \\
\text { no internal } \psi \text {-lines left outside },
\end{array} \\
\bar{t}_{\gamma}^{\prime}=t_{\gamma}^{\prime(0)}, & \text { otherwise. }
\end{array}
$$

Here, $t_{\gamma}^{\prime(2)}$ is an oversubtraction operator which is analogous to the one specified by eqs. $(22 \mathrm{a}-\mathrm{d})$, but treats the auxiliary mass variables $\lambda^{2}, \kappa^{2}$ on an equal footing with external momenta of a proper subgraph in the Taylor series expansion. For instance, for a proper sucgraph corresponding to $H_{4}^{\prime}$ it acts according to

$$
\begin{aligned}
& t^{\prime(2)} H_{4}^{\prime \mathrm{u}}\left(q_{1}, q_{2}, q_{3}, q_{4} ; \lambda^{2}, \kappa^{2}\right)=H_{4}^{\prime \mathrm{u}}\left(q_{1}, \ldots, q_{4} ; \lambda^{2}, \kappa^{2}\right) \underset{q_{i}=0, \lambda^{2}=\kappa^{2}=0}{\mid} \\
& +\left.\lambda^{2}\left[\frac{\partial}{\partial \lambda^{2}} H_{4}^{\prime \mathrm{u}}\left(q_{1}, \ldots, q_{4} ; \lambda^{2}, \kappa^{2}\right)\right]_{q_{i}=0, \lambda^{2}=\kappa^{2}=0}\right|^{2=0}
\end{aligned}
$$




$$
\begin{aligned}
& +\kappa^{2}\left[\frac{\partial}{\partial \kappa^{2}} H_{4}^{\prime \mathrm{u}}\left(q_{1}, \ldots, q_{4} ; \lambda^{2}, \kappa^{2}\right)\right]_{q_{i}=0, \lambda^{2}=\kappa^{2}=0} \\
& +\left.\frac{1}{2} \sum_{k \neq l} q_{k} q_{l}\left[\frac{\partial^{2}}{\partial q_{k} \partial q_{l}} H_{4}^{\prime \mathrm{u}}\left(q_{1}, \ldots, q_{4} ; \lambda^{2}, \kappa^{2}\right)\right]_{q_{i}=0, \lambda^{2}=\kappa^{2}=0}\right|_{k} \\
& +\frac{1}{2} \sum_{k} q_{k}^{2}\left[\frac{\partial^{2}}{\partial q_{k}^{2}} H_{4}^{\prime \mathrm{u}}\left(q_{1}, \ldots, q_{4} ; \lambda^{2}, \kappa^{2}\right)\right]_{q_{i}=0, \lambda^{2}=\kappa^{2}=0} .
\end{aligned}
$$

Now, one may go through the same arguments as described in sect. 2 to obtain $(c f$., eq. (32))

$$
\begin{aligned}
& H_{n}^{\prime}\left(p_{1}, \ldots, p_{n} ; \lambda^{2}, \kappa^{2}\right)=-\sum_{\tau \in \mathrm{T}} \sum_{\Omega_{1} \in \mathrm{M}_{\tau}} \sum_{\Omega_{2} \in \cup(\tau)}\left[\sum_{\gamma \in \Omega_{1}} \Pi\left(-\bar{t}_{\gamma}^{\prime}\right)\right] \\
& \cdot\left[-\left(t_{\tau}^{\prime(2)}-t_{\tau}^{\prime(0)}\right)\right] \cdot\left[\prod_{\gamma^{\prime} \in \Omega_{2}}\left(-t_{\gamma^{\prime}}^{\prime(0)}\right)\right] H_{n}^{\prime \mathrm{u}}\left(p_{1}, \ldots, p_{n} ; \lambda^{2}, \kappa^{2}\right) \\
& \quad+\bar{H}_{n}^{\prime}\left(p_{1}, \ldots, p_{n}, \lambda^{2}, \kappa^{2}\right) .
\end{aligned}
$$

According to simple power counting [9], it is again a simple matter to show that, in the limit of $\mu, \lambda, \kappa, p_{1}, \ldots, p_{n} \ll M$, the oversubtracted function $\bar{H}_{n}\left(p_{1}, \ldots, p_{n}\right.$; $\left.\lambda^{2}, \kappa^{2}\right)$ is at most of order $1 / M^{4}$.

In the $\phi^{\prime}$ field theory with the action (35), we now introduce the following local vertices:

$$
\begin{aligned}
& \tilde{O}_{1}^{\prime}=\frac{1}{2} \rho_{\phi} \int \mathrm{d}^{4} x N\left[\phi^{2}(x)\right], \\
& \widetilde{O}_{2}^{\prime}=-\frac{1}{2} \rho_{\phi} \int \mathrm{d}^{4} x N\left[\phi \partial^{2} \phi(x),\right. \\
& \tilde{O}_{3}^{\prime}=\frac{\rho_{\phi}^{2}}{4 !} \int \mathrm{d}^{4} x N\left[\phi^{4}(x)\right], \\
& \widetilde{O}_{4}^{\prime}=\frac{1}{2} \rho_{\phi} \int \mathrm{d}^{4} x N\left[\phi\left(\partial^{2}\right)^{2} \phi(x)\right], \\
& \widetilde{O}_{5}^{\prime}=-\frac{1}{6} \rho_{\phi}^{2} \int \mathrm{d}^{4} x N\left[\phi^{3} \partial^{2} \phi(x)\right], \\
& \tilde{O}_{6}^{\prime}=\frac{\rho_{\phi}^{3}}{6 !} \int \mathrm{d}^{4} x N\left[\phi^{6}(x)\right],
\end{aligned}
$$

with the Feynman rules for the cerresponding tree vertices as shown in fig. 7. Renormalized proper vertex functions with the local vertices $\widetilde{O}_{i}$ once inserted may be repre sented by the forest formula

$$
\tilde{\Gamma}_{n}^{\prime}\left(\tilde{O}_{i}^{\prime}, p_{1}, \ldots, p_{n} ; \lambda^{2}\right)=\sum_{\Omega} \prod_{\gamma \in \Omega}\left(-t_{\gamma}^{\prime(0)}\right) \widetilde{\Gamma}_{n}^{\prime u}\left(\tilde{O}_{i}^{\prime}, p_{1}, \ldots, p_{n}, \lambda^{2}\right),
$$




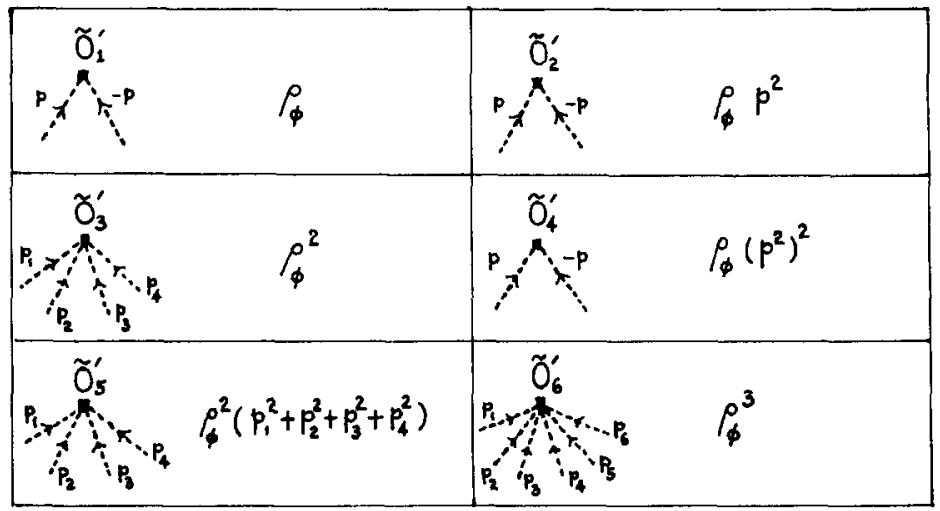

Fig. 7. Tree vertices for $\widetilde{O}_{1}^{\prime}, \widetilde{O}_{2}^{\prime}, \ldots, \widetilde{O}_{6}$.

with $t_{\gamma}^{\prime(0)}$ denoting the minimum (or normal) subtraction operator, defined by appropriate Taylor series in $\lambda^{2}$ and external momenta of the proper subgraph involved. It is then a simple matter to show that the renormalized vertex functions $\widetilde{\Gamma}_{n}^{\prime}\left(\widetilde{O}_{i}^{\prime}, p_{1}, \ldots, p_{n}\right.$ $\left.\lambda^{2}\right)$ satisfy the following normalization conditions:

$$
\begin{aligned}
& \left.\widetilde{\Gamma}_{2}^{\prime}\left(\widetilde{O}_{1}^{\prime}, p,-p ; \lambda^{2}\right)\right|_{p^{2}=\lambda^{2}=0}=\rho_{\phi}, \\
& \left.\widetilde{\Gamma}_{2}^{\prime}\left(\widetilde{O}_{j}^{\prime}, p,-p ; \lambda^{2}\right)\right|_{p^{2}=\lambda^{2}=0}=0, \quad(j=2,3,4,5,6), \\
& {\left.\left[\frac{\partial}{\partial \lambda^{2}} \widetilde{\Gamma}_{2}^{\prime}\left(\tilde{O}_{j}^{\prime}, p,-p ; \lambda^{2}\right)\right]\right|_{p^{2}=\lambda^{2}=0}=0, \quad(j=2,3,4,5,6),} \\
& {\left.\left[\frac{\partial}{\partial p^{2}} \tilde{\Gamma}_{2}^{\prime}\left(\tilde{O}_{2}^{\prime}, p,-p ; \lambda^{2}\right)\right]\right|_{p^{2}=\lambda^{2}=0}=\rho_{\phi},} \\
& \left.\widetilde{\Gamma}_{4}^{\prime}\left(\widetilde{O}_{3}^{\prime}, p_{1}, p_{2}, p_{3}, p_{4} ; \lambda^{2}\right)\right|_{p_{i}=0, \lambda^{2}=0}=\rho_{\phi}^{2}, \\
& {\left.\left[\frac{\partial}{\partial p^{2}} \tilde{\Gamma}_{2}^{\prime}\left(\tilde{O}_{j}^{\prime}, p,-p, \lambda^{2}\right)\right]\right|_{p^{2}=\lambda^{2}=0}=0, \quad(j=3,4,5,6),} \\
& \left.\widetilde{\Gamma}_{4}^{\prime}\left(\tilde{O}_{j}^{\prime}, p_{1}, p_{2}, p_{3}, p_{4}, \lambda^{2}\right)\right|_{p_{i}=0, \lambda^{2}=0}=0, \quad(j=2,4,5,6), \\
& {\left.\left[\frac{\partial^{2}}{\partial\left(\lambda^{2}\right)^{2}} \widetilde{\Gamma}_{2}^{\prime}\left(\tilde{O}_{j}^{\prime}, p,-p ; \lambda^{2}\right)\right]\right|_{p^{2}=\lambda^{2}=0}=0, \quad(j=4,5,6),} \\
& {\left.\left[\frac{\partial}{\partial \lambda^{2}} \frac{\partial}{\partial p^{2}} \widetilde{\Gamma}_{2}^{\prime}\left(\widetilde{O}_{j}^{\prime}, p,-p ; \lambda^{2}\right)\right]\right|_{p^{2}=\lambda^{2}=0}=0,(j=4,5,6),} \\
& {\left.\left[\frac{\partial}{\partial \lambda^{2}} \widetilde{\Gamma}_{4}^{\prime}\left(\widetilde{O}_{j}, p_{1}, p_{2}, p_{3}, p_{4} ; \lambda^{2}\right)\right]\right|_{p_{i}=0, \lambda^{2}=0}=0,(j=4,5,6),}
\end{aligned}
$$




$$
\begin{aligned}
& \left.\tilde{\Gamma}_{2}^{\prime}\left(\tilde{O}_{4}^{\prime}, p,-p, \lambda^{2}=0\right) \underset{p^{2} \rightarrow 0}{=} \rho_{\phi}\left(p^{2}\right)^{2}+\mathrm{O}\left(p^{2}\right)^{3}\right), \\
& \tilde{\Gamma}_{4}^{\prime}\left(\tilde{O}_{5}^{\prime}, p_{1}, p_{2}, p_{3}, p_{4} ; \lambda^{2}=0\right) \underset{p_{i} \rightarrow 0}{=} \rho_{\phi}^{2}\left(p_{1}^{2}+p_{2}^{2}+p_{3}^{2}+p_{4}^{2}\right)+\mathrm{O}\left(p_{i}^{4}\right), \\
& \tilde{\Gamma}_{6}^{\prime}\left(\tilde{O}_{6}^{\prime}, p_{1}, \ldots, p_{6} ; \lambda^{2}=0\right) \underset{p_{i} \rightarrow 0}{=} \rho_{\phi}^{3}+\mathrm{O}\left(p_{i}^{2}\right), \\
& \tilde{\Gamma}_{2}^{\prime}\left(\tilde{O}_{i}^{\prime}, p,-p ; \lambda^{2}=0\right) \underset{p^{2} \rightarrow 0}{=} \mathrm{O}\left(\left(p^{2}\right)^{3}\right), \quad(j=5,6), \\
& \tilde{\Gamma}_{4}^{\prime}\left(\tilde{O}_{j}^{\prime}, p_{1}, p_{2}, p_{3}, p_{4} ; \lambda^{2}=0\right) \underset{p_{i} \rightarrow 0}{=} \mathrm{O}\left(p_{i}^{4}\right), \quad(j=4,6) \\
& \tilde{\Gamma}_{6}^{\prime}\left(\tilde{O}_{j}^{\prime}, p_{1}, \ldots, p_{6} ; \lambda^{2}=0\right) \underset{p_{i} \rightarrow 0}{=} \mathrm{O}\left(p_{i}^{2}\right), \quad(j=4,5) .
\end{aligned}
$$

Let us now introduce the effective couplings $G_{1}^{\prime}, \ldots, G_{6}^{\prime}$ by

$$
\begin{aligned}
& \left(t^{\prime(2)}-t^{\prime(0)}\right) \sum_{\Omega}^{\text {normal }} \prod_{\gamma \in \Omega}\left(-t_{\gamma}^{\prime(0)}\right) H_{2}^{\prime \mathrm{u}}\left(q,-q ; \lambda^{2}, \kappa^{2}\right) \\
& \quad=\rho_{\phi} G_{1}^{\prime}+\rho_{\phi} q^{2} G_{2}^{\prime}+\rho_{\phi}\left(q^{2}\right)^{2} G_{4}^{\prime}, \\
& \left(t^{\prime(2)}-t^{\prime}(0) \sum_{\Omega}^{\text {normal }} \prod_{\gamma \in \Omega}\left(-t_{\gamma}^{\prime(0)}\right) H_{4}^{\prime u}\left(q_{1}, q_{2}, q_{3}, q_{4} ; \lambda^{2}, \kappa^{2}\right)\right. \\
& \quad=\rho_{\phi}^{2} G_{3}^{\prime}+\rho_{\phi}^{2}\left(q_{1}^{2}+q_{2}^{2}+q_{3}^{2}+q_{4}^{2}\right) G_{5}^{\prime}, \\
& \left(t^{\prime(2)}-t^{\prime(0)} \sum_{\Omega}^{\text {normal }} \prod_{\gamma \in \Omega}\left(-t_{\gamma}^{\prime(0)}\right) H_{6}^{\prime u}\left(q_{1}, q_{2}, q_{3}, q_{4}, q_{5}, q_{6} ; \lambda^{2}, \kappa^{2}\right)=\rho_{\phi}^{3} G_{6}^{\prime}\right.
\end{aligned}
$$

Due to the decoupling theorem [4], the constants $G_{1}^{\prime}, \ldots, G_{6}^{\prime}$ are all of order $1 / M^{2}$ and thus we may write

$$
\begin{aligned}
& G_{1}^{\prime}=\frac{1}{M^{2}}\left\{\left(\lambda^{2}\right)^{2} D_{1}+\lambda^{2} \kappa^{2} \bar{D}_{1}+\left(\kappa^{2}\right)^{2} \overline{\bar{D}}_{1}\right\}, \\
& G_{j}^{\prime}=\frac{1}{M^{2}}\left\{\lambda^{2} D_{j}+\kappa^{2} \bar{D}_{j}\right\}, \quad(j=2,3), \\
& G_{k}^{\prime}=\frac{1}{M^{2}} D_{k}, \quad(k=4,5,6),
\end{aligned}
$$

where the dimensionless numbers $D_{1}, \bar{D}_{1}, \overline{\bar{D}}_{1}, D_{2}, \bar{D}_{2}, \ldots, D_{6}$ are independent of 
$\lambda^{2}$ and $\kappa^{2}$ according to our oversubtraction procedure. Upon comparing eqs. $(76 a-c)$ with eqs. $(33 a-c)$ it is evident that

$$
\begin{array}{lll}
G_{1} \equiv G_{4}^{\prime}, & G_{2} \equiv G_{5}^{\prime}, & G_{3} \equiv G_{6}^{\prime}, \\
C_{1} \equiv D_{4}, & C_{2} \equiv D_{5}, & C_{3} \equiv D_{6} .
\end{array}
$$

With this information and eq. (72), it is a simple matter to conclude that

$$
\begin{aligned}
& \Gamma_{n, 0}^{\prime}\left(p_{1}, \ldots, p_{n} ; \lambda^{2}, \kappa^{2}\right)=\widetilde{\Gamma}_{n}^{\prime}\left(p_{1}, \ldots, p_{n} ; \lambda^{2}\right) \\
& \quad+\sum_{i=1}^{6} \widetilde{\Gamma}_{n}^{\prime}\left(\widetilde{O}_{i}^{\prime}, p_{1}, \ldots, p_{n} ; \lambda^{2}\right) G_{i}^{\prime}+O\left(\frac{1}{M^{4}}\right) .
\end{aligned}
$$

Astute readers many note that, due to presence of appropriate powers of $\lambda^{2}$ and $\kappa^{2}$, oversubtractions in the full $(\phi-\psi)^{\prime}$ theory correspond to minimal subtractions as for proper subgraphs with the local vertices $\widetilde{O}_{i}^{\prime}$ once inserted, regardless of the dimension of the operator involved.

Just like ordinary renormalized vertex functions, the functions $\widetilde{\Gamma}_{n}^{\prime}\left(\widetilde{O}_{i}^{\prime}, p_{1}, \ldots, p_{n}\right.$; $\lambda^{2}$ ) also satisfy certain types of Callan-Symanzik equations. Consider the local vertex $\tilde{O}_{1}^{\prime}$. The forest formula

$$
\widetilde{\Gamma}_{n}^{\prime}\left(\widetilde{O}_{1}^{\prime}, p_{1}, \ldots, p_{n} ; \lambda^{2}\right)=\sum_{\Omega} \prod_{\gamma \in \Omega}\left(-t_{\gamma}^{\prime(0)}\right) \widetilde{\Gamma}_{n}^{\prime \mathrm{u}}\left(\tilde{O}_{1}^{\prime}, p_{1}, \ldots, p_{n} ; \lambda^{2}\right),
$$

implies multiplicative renormalization

$$
\tilde{\Gamma}_{n}^{\prime}\left(\tilde{O}_{1}^{\prime}, p_{1}, \ldots, p_{n} ; \lambda^{2}\right)=\widetilde{X}_{11} \widetilde{\Gamma}_{n}^{\prime \prime \prime}\left(\widetilde{O}_{1}^{\prime}, p_{1}, \ldots, p_{n} ; \tilde{m}_{\mathrm{B}}^{2}, \widetilde{\rho}_{\mathrm{B} \phi}, \widetilde{\alpha}_{\mathrm{B}}, \Lambda^{2}\right),
$$

where $\widetilde{\Gamma}_{n}^{\prime u}\left(\widetilde{O}_{1}^{\prime}, p_{1}, \ldots, p_{n} ; \tilde{m}_{\mathrm{B}}^{2}, \tilde{\rho}_{\mathrm{B} \phi}, \widetilde{\alpha}_{\mathrm{B}}, \Lambda^{2}\right)$ denotes the function obtained from the unsubtracted function $\widetilde{\Gamma}_{n}^{\prime \mathrm{u}}\left(\tilde{O}_{1}^{\prime}, p_{1}, \ldots, p_{n} ; \lambda^{2}\right)$ by replacing $\mu^{2}+\lambda^{2}, \rho_{\phi}, \alpha$ with $\tilde{m}_{\mathrm{B}}^{2}, \tilde{\rho}_{\mathrm{B} \phi}, \tilde{\alpha}_{\mathrm{B}}$. In eq. (81), the $\lambda^{2}$ independent constant $\widetilde{X}_{11}$ is equal to 1 in the tree approximation and, in general, involves powers of $\log \Lambda^{2}$ from loop corrections. For the local vertices $\widetilde{O}_{2}^{\prime}, \widetilde{O}_{3}^{\prime}$, the forest formulae for them imply

$$
\begin{aligned}
& \widetilde{\Gamma}_{n}^{\prime}\left(\widetilde{O}_{2}^{\prime}, p_{1}, \ldots, p_{n} ; \lambda^{2}\right)=\widetilde{X}_{22} \widetilde{\Gamma}_{n}^{\prime u}\left(\widetilde{O}_{2}^{\prime}, p_{1}, \ldots, p_{n} ; \tilde{m}_{\mathrm{B}}^{2}, \widetilde{\rho}_{\mathrm{B} \phi}, \widetilde{\alpha}_{\mathrm{B}}, \Lambda^{2}\right) \\
& -\left(\tilde{Y}_{21}^{\prime}+\lambda^{2} \tilde{Y}_{21}\right) \tilde{\Gamma}_{n}^{\prime u}\left(\tilde{O}_{1}^{\prime}, p_{1}, \ldots, p_{n} ; \tilde{m}_{\mathrm{B}}^{2}, \tilde{\rho}_{\mathrm{B} \phi}, \tilde{\alpha}_{\mathrm{B}}, \Lambda^{2}\right) \\
& +\tilde{X}_{23} \widetilde{\Gamma}_{n}^{\prime \mathbf{u}}\left(\tilde{O}_{3}^{\prime}, p_{1}, \ldots, p_{n} ; \tilde{m}_{\mathrm{B}}^{2}, \tilde{\rho}_{\mathrm{B} \phi}, \widetilde{\alpha}_{\mathrm{B}}, \Lambda^{2}\right) \text {, } \\
& \tilde{\Gamma}_{n}^{\prime}\left(\tilde{O}_{3}, p_{1}, \ldots, p_{n} ; \lambda^{2}\right)=\tilde{X}_{33} \widetilde{\Gamma}_{n}^{\prime \prime}\left(\widetilde{O}_{3}^{\prime}, p_{1}, \ldots, p_{n} ; \tilde{m}_{\mathrm{B}}^{2}, \tilde{\rho}_{\mathrm{B} \phi}, \tilde{\alpha}_{\mathrm{B}}, \Lambda^{2}\right) \\
& -\left(\tilde{Y}_{31}^{\prime}+\lambda^{2} \tilde{Y}_{31}\right) \tilde{\Gamma}_{n}^{\prime \mathrm{u}}\left(\tilde{O}_{1}^{\prime}, p_{1}, \ldots, p_{n} ; \tilde{m}_{\mathrm{B}}^{2}, \tilde{\rho}_{\mathrm{B} \phi}, \tilde{\alpha}_{\mathrm{B}}, \Lambda^{2}\right) \\
& +\tilde{X}_{32} \tilde{\Gamma}_{n}^{\prime \mathrm{u}}\left(\tilde{O}_{2}^{\prime}, p_{1}, \ldots, p_{n} ; \tilde{m}_{\mathrm{B}}^{2}, \tilde{\rho}_{\mathrm{B} \phi}, \tilde{\alpha}_{\mathrm{B}}, \Lambda^{2}\right) \text {. }
\end{aligned}
$$


Here, all $\tilde{X}^{\prime}$ s and $\tilde{\widetilde{Y}}^{\prime}$ s are independent of $\lambda^{2}$, and in general $\tilde{Y}_{21}^{\prime}, \widetilde{Y}_{31}^{\prime}$ are $\mathrm{O}\left(\Lambda^{2}\right)$ while $\widetilde{X}_{22}, \widetilde{Y}_{21}, \widetilde{X}_{23}, \widetilde{X}_{33}, \widetilde{Y}_{31}, \widetilde{X}_{32}$ are $\mathrm{O}\left(\left[\log \Lambda^{2}\right]^{\alpha}\right)$ with $\alpha$ being non-negative integers. In the tree approximation,

$$
\tilde{X}_{22}^{(0)}=\tilde{X}_{33}^{(0)}=1, \quad \tilde{Y}_{21}^{(0)}=\tilde{Y}_{21}^{(0)}=\tilde{Y}_{31}^{(0)}=\tilde{Y}_{31}^{(0)}=\tilde{X}_{23}^{(0)}=\tilde{X}_{32}^{(0)}=0 .
$$

Similarly, for the local vertices $\widetilde{O}_{4}^{\prime}, \widetilde{O}_{5}^{\prime}, \widetilde{O}_{6}^{\prime}$, we have $(k, l=4,5,6)$

$$
\begin{aligned}
& \widetilde{\Gamma}_{n}^{\prime}\left(\tilde{O}_{k}^{\prime}, p_{1}, \ldots, p_{n} ; \lambda^{2}\right)=\tilde{X}_{k k} \tilde{\Gamma}_{n}^{\prime \mathrm{u}}\left(\tilde{O}_{k}^{\prime}, p_{1}, \ldots, p_{n} ; \tilde{m}_{\mathrm{B}}^{2}, \tilde{\rho}_{\mathrm{B} \phi}, \tilde{\alpha}_{\mathrm{B}}, \Lambda^{2}\right) \\
& -\left(\tilde{Y}_{k 1}^{\prime \prime}+\tilde{Y}_{k 1}^{\prime} \lambda^{2}+\tilde{Y}_{k 1} \lambda^{4}\right) \tilde{\Gamma}_{n}^{\prime \mathrm{u}}\left(\tilde{O}_{1}^{\prime}, p_{1}, \ldots, p_{n} ; \tilde{m}_{\mathrm{B}}^{2}, \tilde{\rho}_{\mathrm{B} \phi}, \tilde{\alpha}_{\mathrm{B}}, \Lambda^{2}\right) \\
& -\sum_{j=2}^{3}\left(\tilde{Y}_{k j}^{\prime}+\tilde{Y}_{k j} \lambda^{2}\right) \tilde{\Gamma}_{n}^{\prime \mathrm{u}}\left(\tilde{O}_{j}^{\prime}, p_{1}, \ldots, p_{n} ; \tilde{m}_{\mathrm{B}}^{2}, \tilde{\rho}_{\mathrm{B} \phi}, \tilde{\alpha}_{\mathrm{B}}, \Lambda^{2}\right) \\
& \quad+\sum_{\substack{l=4 \\
l \neq k}}^{6} \tilde{X}_{k l} \tilde{\Gamma}_{n}^{\prime \mathrm{u}}\left(\tilde{O}_{l}^{\prime}, p_{1}, \ldots, p_{n} ; \tilde{m}_{\mathrm{B}}^{2}, \tilde{\rho}_{\mathrm{B} \phi}, \tilde{\alpha}_{\mathrm{B}}, \Lambda^{2}\right),
\end{aligned}
$$

where again all $X$ 's and $Y$ 's are independent of $\lambda^{2}$ and

$$
\begin{aligned}
& \tilde{Y}_{k 1}^{\prime \prime} \sim \mathrm{O}\left(\Lambda^{4}\right), \\
& \tilde{Y}_{k 1}^{\prime}, \tilde{Y}_{k 2}^{\prime}, \tilde{Y}_{k 3}^{\prime} \sim \mathrm{O}\left(\Lambda^{2}\right), \\
& \tilde{X}_{k k}, \tilde{Y}_{k 1}, \tilde{Y}_{k 2}, \tilde{Y}_{k 3}, \tilde{X}_{k l} \sim \mathrm{O}\left(\left[\log \Lambda^{2}\right]^{\alpha}\right) .
\end{aligned}
$$

In the tree approximation, $\tilde{X}_{k k}^{(0)}=1(k=4,5,6)$ and all the other $\tilde{X}, \tilde{Y}$ 's vanish.

Let us now define the block diagonal matrix

$$
\widetilde{X}=\left(\begin{array}{llllll}
\widetilde{X}_{11} & 0 & 0 & 0 & 0 & 0 \\
0 & \widetilde{X}_{22} & \widetilde{X}_{23} & 0 & 0 & 0 \\
0 & \widetilde{X}_{32} & \widetilde{X}_{33} & 0 & 0 & 0 \\
0 & 0 & 0 & \widetilde{X}_{44} & \widetilde{X}_{45} & \widetilde{X}_{46} \\
0 & 0 & 0 & \widetilde{X}_{54} & \widetilde{X}_{55} & \widetilde{X}_{56} \\
0 & 0 & 0 & \widetilde{X}_{64} & \widetilde{X}_{65} & \widetilde{X}_{66}
\end{array}\right),
$$

elements of which are at most of order $\left[\log \Lambda^{2}\right]^{\alpha}$ in perturbation theory. If we also define the inverse of the matrix (86) by $\widetilde{X}^{-1}$, we may express eqs. (81), (82a), (82b), (84) in the following forms $(i, j=2,3$ and $k, l=4,5,6)$

$$
\begin{aligned}
& \tilde{\Gamma}_{n}^{\prime \mathrm{u}}\left(\tilde{O}_{1}^{\prime}, p_{1}, \ldots, p_{n} ; \tilde{m}_{\mathrm{B}}^{2}, \tilde{\rho}_{\mathrm{B} \phi}, \tilde{\alpha}_{\mathrm{B}}, \Lambda^{2}\right)=\left(\tilde{X}^{-1}\right)_{11} \tilde{\Gamma}_{n}^{\prime}\left(\tilde{O}_{1}^{\prime}, p_{1}, \ldots, p_{n} ; \lambda^{2}\right) \\
& \tilde{\Gamma}_{n}^{\prime \mathrm{u}}\left(\tilde{O}_{i}^{\prime}, p_{1}, \ldots, p_{n} ; \tilde{m}_{\mathrm{B}}^{2}, \tilde{\rho}_{\mathrm{B} \phi}, \tilde{\alpha}_{\mathrm{B}}, \Lambda^{2}\right)=\left(\tilde{X}^{-1}\right)_{i j}\left\{\tilde{\Gamma}_{n}^{\prime}\left(\tilde{O}_{j}^{\prime}, p_{1}, \ldots, p_{n} ; \lambda^{2}\right)\right. \\
& \left.\quad+\left(\tilde{Y}_{j 1}^{\prime}+\lambda^{2} \tilde{Y}_{j 1}\right)\left(\tilde{X}^{-1}\right)_{11} \tilde{\Gamma}_{n}^{\prime}\left(\tilde{O}_{1}^{\prime}, p_{1}, \ldots, p_{n} ; \lambda^{2}\right)\right\}
\end{aligned}
$$




$$
\begin{aligned}
& \widetilde{\Gamma}_{n}^{\prime \mathrm{u}}\left(\tilde{O}_{k}^{\prime}, p_{1}, \ldots, p_{n} ; \tilde{m}_{\mathrm{B}}^{2}, \tilde{\rho}_{\mathrm{B} \phi} \widetilde{\alpha}_{\mathrm{B}}, \Lambda^{2}\right)=\left(\tilde{X}^{-1}\right)_{k l}\left\{\widetilde{\Gamma}_{n}^{\prime}\left(\widetilde{O}^{\prime}{ }_{l}, p_{1}, \ldots, p_{n} ; \lambda^{2}\right)\right. \\
& +\left(\tilde{Y}_{l 1}^{\prime \prime}+\lambda^{2} \tilde{Y}_{l 1}^{\prime}+\lambda^{4} \tilde{Y}_{l 1}\right)\left(\tilde{X}^{-1}\right)_{11} \tilde{\Gamma}_{n}^{\prime}\left(\widetilde{O}_{1}^{\prime}, p_{1}, \ldots, p_{n} ; \lambda^{2}\right) \\
& +\left(\tilde{Y}_{l i}^{\prime}+\lambda^{2} \widetilde{Y}_{l i}\right)\left(\tilde{X}^{-1}\right)_{i j}\left[\tilde{\Gamma}_{n}^{\prime}\left(\tilde{O}_{j}^{\prime}, p_{1}, \ldots, p_{n} ; \lambda^{2}\right)\right. \\
& \left.\left.+\left(\widetilde{Y}_{j 1}^{\prime}+\lambda^{2} \widetilde{Y}_{j 1}\right) \widetilde{X}_{11}^{-1} \widetilde{\Gamma}_{n}^{\prime}\left(\widetilde{O}_{1}^{\prime}, p_{1}, \ldots, p_{n} ; \lambda^{2}\right)\right]\right\} \text {. }
\end{aligned}
$$

Eqs. $(87 a-c)$ describe the renormalization mixing structure of the local vertices $\widetilde{O}_{i}^{\prime}$. Now we may write down the Callan-Symanzik equations for $\widetilde{\Gamma}_{n}^{\prime}\left(\widetilde{O}_{i}^{\prime}, p_{1}, \ldots, p_{n}\right.$; $\left.\lambda^{2}\right)$, since the left-hand sides of eqs. $(87 \mathrm{a}-\mathrm{c})$ are invariant under the one-parameter variation of varying $\mu^{2}$ for fixed $\tilde{m}_{\mathrm{B}}^{2}, \widetilde{\rho}_{\mathrm{B} \phi}, \widetilde{\alpha}_{\mathrm{B}}, \Lambda^{2}$. For $\widetilde{O}_{1}^{\prime}$, the condition

$$
\mu^{2} \frac{\widetilde{D}}{D \mu^{2}} \widetilde{\Gamma}_{n}^{\prime \mathrm{u}}\left(\tilde{O}_{1}^{\prime}, p_{1}, \ldots, p_{n} ; \tilde{m}_{\mathrm{B}}^{2}, \tilde{\rho}_{\mathrm{B} \phi}, \tilde{\alpha}_{\mathrm{B}}, \Lambda^{2}\right)=0
$$

yields the Callan-Symanzik equation

$$
\begin{aligned}
& \left\{\mu^{2} \frac{\partial}{\partial \mu^{2}}+\widetilde{B}_{\alpha} \alpha \frac{\partial}{\partial \alpha}-\left[\left(1-\widetilde{\Theta}_{1}\right) \mu^{2}-\widetilde{\Theta}_{2} \lambda^{2}\right] \frac{\partial}{\partial \lambda^{2}}+\tilde{\gamma}_{\phi} \rho_{\phi} \frac{\partial}{\partial \rho_{\phi}}+\widetilde{t}_{11}\right\} \\
& \quad \times \widetilde{\Gamma}_{n}^{\prime}\left(\widetilde{O}_{1}^{\prime}, p_{1}, \ldots, p_{n} ; \lambda^{2}\right)=0
\end{aligned}
$$

where

$$
\widetilde{t}_{11}=\widetilde{X}_{11} \mu^{2} \frac{\widetilde{D}}{D \mu^{2}} \tilde{X}_{11}^{-1}
$$

For $\widetilde{O}_{2}^{\prime}$ and $\widetilde{O}_{3}^{\prime}$, the conditions

$$
\mu^{2} \frac{\tilde{D}}{D \mu^{2}} \widetilde{\Gamma}_{n}^{\prime \mathrm{u}}\left(\tilde{O}_{i}^{\prime}, p_{1}, \ldots, p_{n} ; \tilde{m}_{\mathrm{B}}^{2}, \tilde{\rho}_{\mathrm{B} \phi}, \widetilde{\alpha}_{\mathrm{B}}, \Lambda^{2}\right)=0, \quad(i=2,3)
$$

and eq. (89) yields the Callan-Symanzik equations $\left(i, j, j^{\prime}=2,3\right)$

$$
\begin{aligned}
0= & \left\{\mu^{2} \frac{\partial}{\partial \mu^{2}}+\widetilde{B}_{\alpha} \alpha \frac{\partial}{\partial \alpha}-\left[\left(1-\widetilde{\Theta}_{1}\right) \mu^{2}-\widetilde{\Theta}_{2} \lambda^{2}\right] \frac{\partial}{\partial \lambda^{2}}\right. \\
& \left.+\tilde{\gamma}_{\phi} \rho_{\phi} \frac{\partial}{\partial \rho_{\phi}}\right\} \widetilde{\Gamma}_{n}^{\prime}\left(\widetilde{O}_{i}^{\prime}, p_{1}, \ldots, p_{n} ; \lambda^{2}\right)+\widetilde{t}_{i j} \widetilde{\Gamma}_{n}^{\prime}\left(\widetilde{O}_{j}^{\prime}, p_{1}, \ldots, p_{n} ; \lambda^{2}\right) \\
& +\left(\tilde{t}_{i 1}^{\prime}+\lambda^{2} \widetilde{t}_{i 1}\right) \widetilde{\Gamma}_{n}^{\prime}\left(\widetilde{O}_{1}^{\prime}, p_{1}, \ldots, p_{n} ; \lambda^{2}\right),
\end{aligned}
$$

where

$$
\begin{aligned}
& \tilde{t}_{i j} \equiv \tilde{X}_{i j^{\prime}} \mu^{2} \frac{\tilde{D}}{D \mu^{2}}\left(\tilde{X}^{-1}\right)_{j^{\prime} j}, \\
& \widetilde{t}_{i 1}^{\prime}+\lambda^{2} \tilde{t}_{i 1} \equiv \tilde{X}_{i j^{\prime}} \mu^{2} \frac{\tilde{D}}{D \mu^{2}}\left[\left(\tilde{X}^{-1}\right)_{j^{\prime} j}\left(\tilde{Y}_{j 1}^{\prime}+\lambda^{2} \tilde{Y}_{j 1}\right) \tilde{X}_{11}^{-1}\right]-\left(\tilde{Y}_{i 1}^{\prime}+\lambda^{2} \tilde{Y}_{i 1}\right) \tilde{X}_{11}^{-1} \tilde{t}_{11}
\end{aligned}
$$


Similarly, for $\widetilde{O}_{4}^{\prime}, \widetilde{O}_{5}^{\prime}, \widetilde{O}_{6}$, the conditions

$$
\mu^{2} \frac{\tilde{D}}{D \mu^{2}} \tilde{\Gamma}_{n}^{\prime \mathrm{u}}\left(\tilde{O}_{k}, p_{1}, \ldots, p_{n} ; \tilde{m}_{\mathrm{B}}^{2}, \tilde{\rho}_{\mathrm{B} \phi}, \tilde{\alpha}_{\mathrm{B}}, \Lambda^{2}\right)=0, \quad(k=4,5,6)
$$

and eqs. (89) and (92) yield the Callan-Symanzik equations $\left(i, j, j^{\prime}=2,3\right.$ and $\left.k, l, l^{\prime}=4,5,6\right)$

$$
\begin{aligned}
0 & =\left\{\mu^{2} \frac{\partial}{\partial \mu^{2}}+\widetilde{B}_{\alpha} \alpha \frac{\partial}{\partial \alpha}-\left[\left(1-\widetilde{\Theta}_{1}\right) \mu^{2}-\widetilde{\Theta}_{2} \lambda^{2}\right] \frac{\partial}{\partial \lambda^{2}}+\widetilde{\gamma}_{\phi} \rho_{\phi} \frac{\partial}{\partial \rho_{\phi}}\right\} \\
& \times \widetilde{\Gamma}_{n}^{\prime}\left(\widetilde{O}_{k}^{\prime}, p_{1}, \ldots, p_{n} ; \lambda^{2}\right) \\
& +\widetilde{t}_{k l} \widetilde{\Gamma}_{n}^{\prime}\left(\widetilde{O}_{l}^{\prime}, p_{1}, \ldots, p_{n} ; \lambda^{2}\right)+\left(\widetilde{t}_{k i}^{\prime}+\lambda^{2} \widetilde{t}_{k i}\right) \widetilde{\Gamma}_{n}^{\prime}\left(\widetilde{O}_{i}^{\prime}, p_{1}, \ldots, p_{n} ; \lambda^{2}\right) \\
& +\left(\widetilde{t}_{k 1}^{\prime \prime}+\lambda^{2} \widetilde{t}_{k 1}^{\prime}+\lambda^{4} \widetilde{t}_{k 1}\right) \widetilde{\Gamma}_{n}^{\prime}\left(\widetilde{O}_{1}, p_{1}, \ldots, p_{n} ; \lambda^{2}\right),
\end{aligned}
$$

where

$$
\begin{aligned}
& \tilde{t}_{k l} \equiv \tilde{X}_{k l^{\prime}} \mu^{2} \frac{\tilde{D}}{D \mu^{2}}\left(\tilde{X}^{-1}\right)_{l^{\prime} l} \\
& \tilde{t}_{k i}^{\prime}+\lambda^{2} \widetilde{t}_{k i} \equiv \tilde{X}_{k l^{\prime} \mu^{2}} \frac{\tilde{D}}{D \mu^{2}}\left[\left(\tilde{X}^{-1}\right)_{l^{\prime} l}\left(\tilde{Y}_{l j}^{\prime}+\lambda^{2} \widetilde{Y}_{l j}\right)\left(\tilde{X}^{-1}\right)_{j i}\right] \\
& -\left(\tilde{Y}_{k j}^{\prime}+\lambda^{2} \tilde{Y}_{k j}\right)\left(\tilde{X}^{-1}\right)_{j j^{\prime}}, \tilde{t}_{j^{\prime} i} \\
& \tilde{t}_{k 1}^{\prime \prime}+\lambda^{2} \widetilde{t}_{k 1}^{\prime}+\lambda^{4} \widetilde{t}_{k 1} \equiv \widetilde{X}_{k l^{\prime}} \mu^{2} \frac{\widetilde{D}}{D \mu^{2}}\left[\left(\tilde{X}^{-1}\right)_{l^{\prime} l}\left(\tilde{Y}_{l 1}^{\prime \prime}+\lambda^{2} \tilde{Y}_{l 1}^{\prime}+\lambda^{4} \widetilde{Y}_{l 1}\right) \tilde{X}_{11}^{-1}\right] \\
& +\widetilde{X}_{k l^{\prime} \mu^{2}} \frac{\widetilde{D}}{D \mu^{2}}\left[\left(\tilde{X}^{-1}\right)_{l^{\prime} l}\left(\tilde{Y}_{l j}^{\prime}+\lambda^{2} \tilde{Y}_{l j}\right)\left(\tilde{X}^{-1}\right)_{j i}\left(\tilde{Y}_{i 1}^{\prime}+\lambda^{2} \tilde{Y}_{i 1}\right) \tilde{X}_{11}^{-1}\right] \\
& -\left(\tilde{Y}_{k j}^{\prime}+\lambda^{2} \tilde{Y}_{k j}\right)\left(\tilde{X}^{-1}\right)_{j j^{\prime}}\left(\tilde{t}_{j^{\prime} 1}^{\prime}+\lambda^{2} \tilde{t}_{j^{\prime} 1}\right)-\left(\tilde{Y}_{k 1}^{\prime \prime}+\lambda^{2} \tilde{Y}_{k 1}^{\prime}+\lambda^{4} \tilde{Y}_{k 1}\right) \tilde{X}_{11}^{-1} \tilde{t}_{11} \\
& -\left(\tilde{Y}_{k j}^{\prime}+\lambda^{2} \widetilde{Y}_{k j}\right)\left(\tilde{X}^{-1}\right)_{j i}\left(\tilde{Y}_{i 1}^{\prime}+\lambda^{2} \tilde{Y}_{i 1}\right) \tilde{X}_{11}^{-1} \tilde{t}_{11}
\end{aligned}
$$

Since eqs. (89), (92), (95) are partial differential equations for renormalized vertex functions $\widetilde{\Gamma}_{n}^{\prime}\left(\widetilde{O}_{i}^{\prime}, p_{1}, \ldots, p_{n} ; \lambda^{2}\right)(i=1,2, \ldots, 6)$, the constants $\widetilde{t}_{i j}, \widetilde{t}_{i j}^{\prime}, \tilde{t}_{i j}^{\prime \prime}$ are expected to be finite (i.e. $\Lambda^{2}$ independent). In fact, from the normalization conditions $(75 \mathrm{a}-\mathrm{p})$, one can easily conclude that

$$
\tilde{t}_{i 1}^{\prime}=\widetilde{t}_{k i}^{\prime}=\widetilde{t}_{k 1}^{\prime \prime}=\tilde{t}_{k 1}^{\prime}=0, \quad(i=2,3 \text { and } k=4,5,6) .
$$

If we factor out the wave-function constant by writing

$$
\begin{aligned}
& \widetilde{\Gamma}_{n}^{\prime}\left(\widetilde{O}_{i}^{\prime} p_{1}, \ldots, p_{n} ; \lambda^{2}=\rho_{\phi}^{n / 2} \widetilde{V}_{n}\left(\widetilde{O}_{i}^{\prime}, p_{1}, \ldots, p_{n} ; \lambda^{2}\right),\right. \\
& \widetilde{V}_{n}\left(\widetilde{O}_{i}^{\prime}, p_{1}, \ldots, p_{n} ; \lambda^{2}\right)=\left.\widetilde{\Gamma}_{n}^{\prime}\left(\widetilde{O}_{i}^{\prime}, p_{1}, \ldots, p_{n} ; \lambda^{2}\right)\right|_{\rho_{\phi}=1},
\end{aligned}
$$


the Callan-Symanzik equations (89), (92), (95) can be written as

$$
\begin{aligned}
& {\left[\left\{\mu^{2} \frac{\partial}{\partial \mu^{2}}+\widetilde{B}_{\alpha} \alpha \frac{\partial}{\partial \alpha}-\left[\left(1-\widetilde{\Theta}_{1}\right) \mu^{2}-\widetilde{\Theta}_{2} \lambda^{2}\right] \frac{\partial}{\partial \lambda^{2}}+\frac{1}{2} n \widetilde{\gamma}_{\phi}\right\} \delta_{i j}\right.} \\
& \left.\quad+\widetilde{T}_{i j}\right] \widetilde{V}_{n}\left(\widetilde{O}_{j}^{\prime}, p_{1}, \ldots, p_{n} ; \lambda^{2}\right)=0
\end{aligned}
$$

with

$$
\widetilde{T}=\left(\begin{array}{llllll}
\widetilde{t}_{11} & 0 & 0 & 0 & 0 & 0 \\
\lambda^{2} \widetilde{t}_{21} & \widetilde{t}_{22} & \widetilde{t}_{23} & 0 & 0 & 0 \\
\lambda^{2} \widetilde{t}_{31} & \widetilde{t}_{32} & \widetilde{t}_{33} & 0 & 0 & 0 \\
\lambda^{4} \widetilde{t}_{41} & \lambda^{2} \widetilde{t}_{42} & \lambda^{2} \widetilde{t}_{43} & \widetilde{t}_{44} & \widetilde{t}_{45} & \widetilde{t}_{46} \\
\lambda^{4} \widetilde{t}_{51} & \lambda^{2} \widetilde{t}_{52} & \lambda^{2} \widetilde{t}_{53} & \widetilde{t}_{54} & \widetilde{t}_{55} & \widetilde{t}_{56} \\
\lambda^{4} \widetilde{t}_{61} & \lambda^{2} \widetilde{t}_{62} & \lambda^{2} \widetilde{t}_{63} & \widetilde{t}_{64} & \widetilde{t}_{65} & \widetilde{t}_{66}
\end{array}\right)
$$

The elements of the triangular block matrix $\tilde{T}$ may be explicitly expressed in terms of appropriate derivatives of $\widetilde{V}_{n}\left(\widetilde{O}_{i}^{\prime}, p_{1}, \ldots, p_{n} ; \lambda^{2}\right)$. Equivalently, the constants $\widetilde{t}_{i j}$ (and $\tilde{a}_{i j}$, which will be used later) may be read off from the following double Taylor series $(i=2,3$ and $k=4,5,6)$

$$
\begin{aligned}
& \widetilde{V}_{2}\left(\widetilde{O}_{1}^{\prime}, p,-p ; \lambda^{2}\right)_{p^{2}, \lambda^{2} \rightarrow 0}^{=} 1+\frac{\lambda^{2}}{\mu^{2}}\left(\frac{\widetilde{\gamma}_{\phi}+\tilde{t}_{11}}{1-\widetilde{\Theta}_{1}}\right)+\frac{p^{2}}{\mu^{2}}\left(\frac{\tilde{a}_{12}}{1-\widetilde{\Theta}_{1}}\right)+\ldots \\
& \widetilde{V}_{4}\left(\tilde{O}_{1}^{\prime}, p_{1}, p_{2}, p_{3}, p_{4} ; \lambda^{2}\right)_{p_{i} \rightarrow 0}=\frac{1}{\lambda^{2} \rightarrow 0}\left(\frac{\tilde{a}_{13}}{1-\widetilde{\Theta}_{1}}\right)+\ldots, \\
& \widetilde{V}_{2}\left(\widetilde{O}_{2}^{\prime}, p,-p ; \lambda^{2}\right)_{p^{2}, \lambda^{2} \rightarrow 0}^{=} \frac{1}{2} \frac{\lambda^{4}}{\mu^{2}}\left(\frac{\widetilde{t}_{21}}{1-\widetilde{\Theta}_{1}}\right)+p^{2}\left[1+\frac{\lambda^{2}}{\mu^{2}}\left(\frac{\widetilde{\gamma}_{\phi}+\widetilde{t}_{22}}{1-\widetilde{\Theta}_{1}}\right)\right] \\
& +\frac{1}{2} \frac{\left(p^{2}\right)^{2}}{\mu^{2}}\left(\frac{\tilde{a}_{24}}{1-\widetilde{\Theta}_{1}}\right)+\ldots \\
& \widetilde{V}_{4}\left(\tilde{O}_{2}^{\prime}, p_{1}, p_{2}, p_{3}, p_{4} ; \lambda^{2}\right)_{p_{i} \rightarrow 0, \lambda^{2} \rightarrow 0}^{=} \frac{\lambda^{2}}{\mu^{2}}\left(\frac{\tilde{t}_{23}}{1-\widetilde{\Theta}_{1}}\right) \\
& +\frac{\lambda^{2}}{\mu^{4}}\left(p_{1}^{2}+p_{2}^{2}+p_{3}^{2}+p_{4}^{2}\right)\left(\frac{\tilde{a}_{25}}{1-\widetilde{\Theta}_{1}}\right)+\ldots \\
& \widetilde{V}_{2}\left(\widetilde{O}_{3}^{\prime}, p,-p ; \lambda^{2}\right)_{p^{2}, \lambda^{2} \rightarrow 0}^{=} \frac{1}{2} \frac{\lambda^{4}}{\mu^{2}}\left(\frac{\widetilde{t}_{31}}{1-\widetilde{\Theta}_{1}}\right)+p^{2} \frac{\lambda^{2}}{\mu^{2}}\left(\frac{\tilde{t}_{32}}{1-\widetilde{\Theta}_{1}}\right) . \\
& +\frac{1}{2}\left(p^{2}\right)^{2}\left(\frac{\widetilde{a}_{34}}{1-\widetilde{\Theta}_{1}}\right)+\ldots
\end{aligned}
$$




$$
\begin{aligned}
& \tilde{V}_{4}\left(\widetilde{O}_{3}^{\prime}, p_{1}, p_{2}, p_{3}, p_{4} ; \lambda^{2}\right)_{p_{i} \rightarrow 0, \lambda^{2} \rightarrow 0}^{=} 1+\frac{\lambda^{2}}{\mu^{2}}\left(\frac{2 \widetilde{\gamma}_{\phi}+\widetilde{t}_{33}}{1-\widetilde{\Theta}_{1}}\right) \\
& +\frac{\lambda^{2}}{\mu^{4}}\left(p_{1}^{2}+p_{2}^{2}+p_{3}^{2}+p_{4}^{2}\right)\left(\frac{\tilde{a}_{35}}{1-\widetilde{\Theta}_{1}}\right)+\ldots \\
& \tilde{V}_{6}\left(\widetilde{O}_{i}^{\prime}, p_{1}, \ldots, p_{6} ; \lambda^{2}\right)_{p_{i} \rightarrow 0, \lambda^{2} \rightarrow 0} \frac{1}{\mu^{2}}\left(\frac{\tilde{a}_{i 6}}{1-\widetilde{\Theta}_{1}}\right)+\ldots, \quad(i=2,3) \\
& \tilde{V}_{2}\left(\tilde{O}_{k}^{\prime}, p,-p ; \lambda^{2}\right)_{p^{2}, \lambda^{2} \rightarrow 0}^{=} \frac{1}{3} \frac{\lambda^{6}}{\mu^{3}}\left(\frac{\tilde{t}_{k 1}}{1-\widetilde{\Theta}_{1}}\right)+\frac{1 \lambda^{4} p^{2}}{2 \mu^{2}}\left(\frac{\tilde{t}_{k 2}}{1-\widetilde{\Theta}_{1}}\right) \\
& +\delta_{k 4}\left(p^{2}\right)^{2}\left[1+\frac{\lambda^{2}}{\mu^{2}}\left(\frac{\widetilde{\gamma}_{\phi}+\tilde{t}_{44}}{1-\widetilde{\Theta}_{1}}\right)\right]+\left(1-\delta_{k 4}\right) \frac{\left(p^{2}\right)^{2} \lambda^{2}}{\mu^{2}}\left(\frac{\tilde{t}_{k 4}}{1-\widetilde{\Theta}_{1}}\right)+\ldots, \\
& (k=4,5,6) \text {, } \\
& \widetilde{V}_{4}\left(\tilde{O}_{k}^{\prime}, p_{1}, p_{2}, p_{3}, p_{4} ; \lambda^{2}\right)_{p_{i} \rightarrow 0, \lambda^{2} \rightarrow 0}^{=} \frac{1}{2} \frac{\lambda^{4}}{\mu^{2}}\left(\frac{\widetilde{t}_{k 3}}{1-\widetilde{\Theta}_{1}}\right) \\
& +\delta_{k 5}\left(p_{1}^{2}+p_{2}^{2}+p_{3}^{2}+p_{4}^{2}\right)\left[1+\frac{\lambda^{2}}{\mu^{2}}\left(\frac{2 \widetilde{\gamma}_{\phi}+\widetilde{t}_{55}}{1-\widetilde{\Theta}_{1}}\right)\right] \\
& +\left(1-\delta_{k 5}\right) \frac{\lambda^{2}}{\mu^{2}}\left(p_{1}^{2}+p_{2}^{2}+p_{3}^{2}+p_{4}^{2}\right)\left(\frac{\widetilde{t}_{k 5}}{1-\widetilde{\Theta}_{1}}\right)+\ldots, \quad(k=4,5,6) \\
& \tilde{V}_{6}\left(\tilde{O}_{k}^{\prime}, p_{1}, \ldots, p_{6} ; \lambda^{2}\right)_{p_{i} \rightarrow 0, \lambda^{2} \rightarrow 0}^{=} \delta_{k 6}\left[1+\frac{\lambda^{2}}{\mu^{2}}\left(\frac{3 \tilde{\gamma}_{\phi}+\tilde{t}_{66}}{1-\widetilde{\Theta}_{1}}\right)\right] \\
& +\left(1-\delta_{k 6}\right) \frac{\lambda^{2}}{\mu^{2}}\left(\frac{\widetilde{t}_{k 6}}{1-\widetilde{\Theta}_{1}}\right)+\ldots .
\end{aligned}
$$

Here, the constants $\tilde{t}_{i j}$, $\tilde{a}_{i j}$ are evidently functions of $\alpha$ only.

We are now ready to derive the Callan-Symanzik equations satisfied by the couplings $G_{i}^{\prime}$, which have the general structure of eqs. $(77 \mathrm{a}-\mathrm{c})$. Inserting the factorized form (79) into the Callan-Symanzik equation, (65), we obtain

$$
\begin{aligned}
0= & \left\{\mu^{2} \frac{\partial}{\partial \mu^{2}}+B_{\alpha} \alpha \frac{\partial}{\partial \alpha}-\left[\mu^{2}\left(1-\Theta_{1}\right)-\lambda^{2} \Theta_{2}-\kappa^{2} \Theta_{3}\right] \frac{\partial}{\partial \lambda^{2}}+\frac{1}{2} n \gamma_{\phi}\right\} \widetilde{V}_{n}\left(p_{1} \ldots, p_{n} ; \lambda^{2}\right) \\
& +\sum_{i=1}^{6} \widetilde{V}_{n}\left(\widetilde{O}_{i}^{\prime}, p_{1}, \ldots, p_{n} ; \lambda^{2}\right)\left\{\mu^{2} \frac{\partial}{\partial \mu^{2}}+B_{\alpha} \alpha \frac{\partial}{\partial \alpha}+B_{\beta} \beta \frac{\partial}{\partial \beta}+B_{\gamma} \gamma \frac{\partial}{\partial \gamma}\right. \\
& \left.-\left[\mu^{2}\left(1-\Theta_{1}\right)-\lambda^{2} \Theta_{2}-\kappa^{2} \Theta_{3}\right] \frac{\partial}{\partial \lambda^{2}}+\left[\lambda^{2} \Omega_{2}+\kappa^{2} \Omega_{3}\right] \frac{\partial}{\partial \kappa^{2}}\right\} G_{i}^{\prime}
\end{aligned}
$$




$$
\begin{aligned}
& +\sum_{i=1}^{6} G_{i}^{\prime}\left\{\mu^{2} \frac{\partial}{\partial \mu^{2}}+B_{\alpha} \alpha \frac{\partial}{\partial \alpha}-\left[\mu^{2}\left(1-\Theta_{1}\right)-\lambda^{2} \Theta_{2}-\kappa^{2} \Theta_{3}\right] \frac{\partial}{\partial \lambda^{2}}\right. \\
& \left.+\frac{1}{2} n \gamma_{\phi}\right\} \widetilde{V}_{n}\left(\tilde{O}_{i}, p_{1}, \ldots, p_{n} ; \lambda^{2}\right) .
\end{aligned}
$$

If we use the Callan-Symanzik equations (51) and (99) for the functions $\widetilde{V}_{n}\left(p_{1}, \ldots, p_{n}\right.$; $\left.\lambda^{2}\right)$ and $\widetilde{V}_{n}\left(\widetilde{O}_{i}, p_{1}, \ldots, p_{n} ; \lambda^{2}\right)$, respectively, eq. (102) can be written in the form

$$
\begin{aligned}
0= & \left\{\left(\Delta B_{\alpha}\right) \alpha \frac{\partial}{\partial \alpha}+\mu^{2}\left(\Delta \Theta_{1}\right) \frac{\partial}{\partial \lambda^{2}}+\left(\Delta \Theta_{2}\right) \lambda^{2} \frac{\partial}{\partial \lambda^{2}}+\Theta_{3} \kappa^{2} \frac{\partial}{\partial \lambda^{2}}\right. \\
& \left.+\frac{1}{2} n \Delta \gamma_{\phi}\right\} \widetilde{V}_{n}\left(p_{1}, \ldots, p_{n} ; \lambda^{2}\right)+\sum_{i=1}^{6} \widetilde{V}_{n}\left(\tilde{O}_{i}^{\prime} p_{1}, \ldots, p_{n} ; \lambda^{2}\right) \\
& \times\left\{\mu^{2} \frac{\partial}{\partial \mu^{2}}+B_{\alpha} \alpha \frac{\partial}{\partial \alpha}+B_{\beta} \beta \frac{\partial}{\partial \beta}+B_{\gamma} \gamma \frac{\partial}{\partial \gamma}\right. \\
& \left.-\left[\mu^{2}\left(1-\Theta_{1}\right)-\lambda^{2} \Theta_{2}-\kappa^{2} \Theta_{3}\right] \frac{\partial}{\partial \lambda^{2}}+\left[\lambda^{2} \Omega_{2}+\kappa^{2} \Omega_{3}\right] \frac{\partial}{\partial \kappa^{2}}\right\} G_{i}^{\prime} \\
& +\sum_{i=1}^{6} C_{i}\left\{\delta _ { i j } \left[\left(\Delta \beta_{\alpha}\right) \alpha \frac{\partial}{\partial \alpha}+\left(\Delta \Theta_{1}\right) \mu^{2} \frac{\partial}{\partial \lambda^{2}}+\left(\Delta \Theta_{2}\right) \lambda^{2} \frac{\partial}{\partial \lambda^{2}}\right.\right. \\
& \left.\left.+\Theta_{3} \kappa^{2} \frac{\partial}{\partial \lambda^{2}}+\frac{1}{2} n \Delta \gamma_{\phi}\right]-\widetilde{T}_{i j}\right\} \widetilde{V}_{n}\left(\widetilde{O}_{j}^{\prime}, p_{1}, \ldots, p_{n} ; \lambda^{2}\right),
\end{aligned}
$$

where we have defined

$$
\begin{array}{ll}
\Delta B_{\alpha}=B_{\alpha}-\widetilde{B}_{\alpha}, & \Delta \gamma_{\phi}=\gamma_{\phi}-\widetilde{\gamma}_{\phi}, \\
\Delta \Theta_{1}=\Theta_{1}-\widetilde{\Theta}_{1}, & \Delta \Theta_{2}=\Theta_{2}-\widetilde{\Theta}_{2} .
\end{array}
$$

We now note that all the effective couplings $C_{i}$ are of order $1 / M^{2}$, and thus it is clear that, for eq. (103) to be true, we must have

$$
\Delta B_{\alpha}, \Delta \Theta_{1}, \Delta \Theta_{2}, \Theta_{3}, \Delta \gamma_{\phi} \sim \mathrm{O}\left(\frac{1}{M^{2}}\right)
$$

Actually, the conclusion (105) is a direct consequence following from the expressions given for $\mathrm{B}_{\alpha}, \gamma_{\phi}, \Theta_{1}, \Theta_{2}, \Theta_{3}, \widetilde{\mathrm{B}}_{\alpha}, \widetilde{\gamma}_{\phi}, \widetilde{\Theta}_{1}, \widetilde{\Theta}_{2}$ in eqs. (52) and (66), and the factorization given in eq. (79). Hence, ignoring terms of $\mathrm{O}\left(1 / M^{4}\right)$, eq. (103) becomes

$$
\begin{aligned}
& \sum_{i, j=1}^{6} \widetilde{V}_{n}\left(\tilde{O}_{i}^{\prime}, p_{1}, \ldots, p_{n} ; \lambda^{2}\right)\left\{\left[\mu^{2} \frac{\partial}{\partial \mu^{2}}+\widetilde{B}_{\alpha} \alpha \frac{\partial}{\partial \alpha}+B_{\beta} \beta \frac{\partial}{\partial \beta}+B_{\gamma} \gamma \frac{\partial}{\partial \gamma}\right.\right. \\
& \left.\left.\quad-\left[\mu^{2}\left(1-\widetilde{\Theta}_{1}\right)-\lambda^{2} \widetilde{\Theta}_{2}\right] \frac{\partial}{\partial \lambda^{2}}+\left(\lambda^{2} \Omega_{2}+\kappa^{2} \Omega_{3}\right) \frac{\partial}{\partial \kappa^{2}}\right] \delta_{i j}-\tilde{T}_{i j}^{*}\right\} G_{j}^{\prime}
\end{aligned}
$$




$$
\begin{aligned}
& =-\left[\left(\Delta B_{\alpha}\right) \alpha \frac{\partial}{\partial \alpha}+\left(\Delta \Theta_{1}\right) \mu^{2} \frac{\partial}{\partial \lambda^{2}}+\left(\Delta \Theta_{2}\right) \lambda^{2} \frac{\partial}{\partial \lambda^{2}}+\Theta_{3} \kappa^{2} \frac{\partial}{\partial \lambda^{2}}\right. \\
& \left.+\frac{1}{2} n \Delta \gamma_{\phi}\right] \tilde{V}_{n}\left(p_{1}, \ldots, p_{n} ; \lambda^{2}\right),
\end{aligned}
$$

where $\tilde{T}_{i j}^{*}=\widetilde{T}_{i j}$.

Eq. (106) is not in a desired form yet for the following two reasons:

(i) eq. (106) involves the constants like $B_{\beta}, B_{\gamma}, \Omega_{2}, \Delta B_{\alpha}, \Delta \gamma_{\phi}, \Delta \Theta_{1}, \Delta \Theta_{2}$ which are not completely determined by the pure light-particle theory;

(ii) eq. (106) involves the cumbersome momentum-dependent functions like $\widetilde{V}_{n}\left(\widetilde{O}_{i}, p_{1}, \ldots, p_{n} ; \lambda^{2}\right)$ and $\widetilde{V}_{n}\left(p_{1}, \ldots, p_{n} ; \lambda^{2}\right)$. In the first place, let us consider the functions $B_{\beta}, B_{\gamma}, \Omega_{2}, \Omega_{3}$, which are specified by the expressions $(66 \mathrm{f}-\mathrm{h}, \mathrm{j}, \mathrm{k})$. Now, according to simple power counting, one may observe that, in the limit of $\mu, \lambda, \kappa<<M$,

$$
\begin{aligned}
& {\left[\frac{\partial}{\partial \lambda^{2}} \frac{\partial}{\partial P^{2}} V_{0,2}\left(P,-P ; \lambda^{2}, \kappa^{2}\right)\right]_{P^{2}=M^{2}, \lambda^{2}=\kappa^{2}=0} \sim \mathrm{O}\left(\frac{1}{M^{2}}\right),} \\
& {\left[\frac{\partial^{2}}{\partial\left(\lambda^{2}\right)^{2}} V_{0,2}\left(P,-P ; \lambda^{2}, \kappa^{2}\right)\right]_{P^{2}=M^{2}, \lambda^{2}=\kappa^{2}=0} \sim \mathrm{O}(1),} \\
& {\left[\frac{\partial}{\partial \lambda^{2}} \frac{\partial}{\partial \kappa^{2}} V_{0,2}\left(P,-P ; \lambda^{2}, \kappa^{2}\right)\right]_{P^{2}=M^{2}, \lambda^{2}=\kappa^{2}=0} \sim \mathrm{O}\left(\frac{1}{M^{2}}\right),} \\
& {\left[\frac{\partial}{\partial \lambda^{2}} V_{0,4}\left(P_{1}, \ldots, P_{4} ; \lambda^{2}, \kappa^{2}\right)\right] \quad \lambda^{2}=\kappa^{2}=0, P_{i}^{2}=M^{2}} \\
& \left(P_{1}+P_{2}\right)^{2}=\left(P_{2}+P_{3}\right)^{2}=\left(P_{1}+P_{3}\right)^{2}={ }_{3}^{4} M^{2} \\
& {\left[\frac{\partial}{\partial \lambda^{2}} V_{2,2}\left(p_{1}, p_{2}, P_{1}, P_{2} ; \lambda^{2}, \kappa^{2}\right)\right]_{p_{i}=0, P^{2}=M^{2}, \lambda^{2}=\kappa^{2}=0} \sim \mathrm{O}(1) .}
\end{aligned}
$$

Inserting the behaviors $(107 \mathrm{a}-\mathrm{e})$ into eq. (66), we conclude that

$$
\gamma_{\psi} \sim \mathrm{O}\left(\frac{1}{M^{2}}\right), \quad \Omega_{2} \sim \mathrm{O}(1), \quad \Omega_{3} \sim \mathrm{O}\left(\frac{1}{M^{2}}\right), \quad B_{\beta} \sim \mathrm{O}\left(\frac{1}{M^{2}}\right), \quad B_{\gamma} \sim \mathrm{O}(1) .
$$

Thus, in the left-hand side of eq. (105), the derivative $B_{\beta} \beta \partial / \partial \beta+\Omega_{3} \kappa^{2} \partial / \partial \kappa^{2}$ may be safely ignored in our analysis which is valid only up to order $1 / M^{2}$. As for $B_{\gamma}$ and $\Omega_{2}$, we have to use the idea of factorization once more with the vertex functions, $\Gamma_{2,2}^{\prime}\left(p_{1}, p_{2}, P_{1}, P_{2} ; \lambda^{2}, \kappa^{2}\right)$ and $\left(\partial^{2} / \partial\left(\lambda^{2}\right)^{2}\right) \Gamma_{0,2}^{\prime}\left(P,-P ; \lambda^{2}, \kappa^{2}\right)$, in the limit of $P_{1}, P_{2}, P, M<<p_{1}, p_{2}, \mu, \lambda, \kappa^{\star}$. In appendix A, we derive the factorizations

ॠ More precisely, we are considering the limit of $\left|P_{1}^{2}\right|^{1 / 2},\left|P_{2}^{2}\right|^{1 / 2}, M \gg>\mu, \kappa,\left|p_{1 \nu}\right|,\left|p_{2 \nu}\right|$, $(\nu=1,2,3,4)$ for $\Gamma_{22}^{\prime}$, and the limit of $\left|P^{2}\right|^{1 / 2}, M>>\mu, \lambda, k$ for $\left(\partial^{2} / \partial\left(\lambda^{2}\right)^{2}\right) \Gamma_{0,2}^{\prime}$ 
(i) $\Gamma_{2,2}^{\prime}\left(p_{1}, p_{2}, P_{1}, P_{2} ; \lambda^{2}, \kappa^{2}\right)$ in the limit of $P_{1}, P_{2}, M \gg p_{1}, p_{2}, \mu, \lambda, \kappa$ :

$$
\begin{aligned}
& \Gamma_{2,2}^{\prime}\left(p_{1}, p_{2}, P_{1}, P_{2} ; \lambda^{2} \kappa^{2}\right)=-i \gamma \widetilde{\Gamma}_{2}^{\prime}\left({ }_{2}^{1} N\left[\phi^{2}(x=0)\right], p_{1}, p_{2} ; \lambda^{2}\right) \\
& \quad+\mathrm{O}\left(\frac{1}{M}\right),
\end{aligned}
$$

(ii) $\frac{\partial^{2}}{\partial\left(\lambda^{2}\right)^{2}} \Gamma_{0,2}^{\prime}\left(P,-P ; \lambda^{2}, \kappa^{2}\right)$ in the limit of $P, M \gg \lambda, \kappa, \mu$ :

$$
\frac{\partial^{2}}{\partial\left(\lambda^{2}\right)^{2}} \Gamma_{0,2}^{\prime}\left(P,-P ; \lambda^{2}, \kappa^{2}\right)=-i \gamma \frac{\partial^{2}}{\partial\left(\lambda^{2}\right)^{2}} \widetilde{\Gamma}_{0,0}^{\prime}\left({ }_{2}^{1} N\left[\phi^{2}(x=0)\right] ; \lambda^{2}\right)+\mathrm{O}\left(\frac{1}{M}\right)
$$

(Here, $\widetilde{\Gamma}_{0}^{\prime}\left(\frac{1}{2} N\left[\phi^{2}(x=0)\right] ; \lambda^{2}\right)$ denotes the vacuum matrix element of the local operator $\frac{1}{2} N\left[\phi^{2}(x=0)\right]$. See appendix A for details.) Inserting the result (109) into eq. (66k), we obtain

$$
\begin{aligned}
& -i B_{\gamma} \gamma-i \widetilde{\gamma}_{\phi} \gamma=-i \gamma\left(1-\widetilde{\Theta}_{1}\right) \mu^{2}\left[\frac{\partial}{\partial \lambda^{2}} \widetilde{V}_{2}\left(\frac{1}{2} N\left[\phi^{2}(x=0)\right], p_{1}, p_{2} ; \lambda^{2}\right)\right] \underset{p_{i}=0, \lambda^{2}=0}{\mid} \\
& +\mathrm{O}\left(\frac{1}{M}\right)=-i \gamma\left(\tilde{\gamma}_{\phi}+\tilde{t}_{11}\right)+\mathrm{O}\left(\frac{1}{M}\right),
\end{aligned}
$$

where $\tilde{t}_{11}$ can be obtained from eq. (101a). From eq. (111), it is clear that

$$
B_{\gamma}=\tilde{t}_{11}+\mathrm{O}\left(\frac{1}{M}\right)
$$

On the other hand, eqs. (110) and (66g) yield the expression

$$
\Omega_{2}=\gamma \widetilde{\Omega}+\mathrm{O}\left(\frac{1}{M}\right)
$$

with

$$
\widetilde{\Omega}=\left.\left(1-\tilde{\Theta}_{1}\right) \mu^{2} \frac{\partial^{2}}{\partial\left(\lambda^{2}\right)^{2}} \tilde{V}_{0,0}\left(\frac{1}{2} N\left[\phi^{2}(x=0)\right] ; \lambda^{2}\right){ }_{\lambda^{2}=0}\right|_{1}
$$

With this information and eq. (106), it is a simple matter to obtain the Callan-Symmanzik equation (which is valid up to $O\left(1 / M^{2}\right)$ ):

$$
\begin{gathered}
\sum_{i, j=1}^{6} \widetilde{V}_{n}\left(\widetilde{O}_{i}^{\prime}, p_{1}, \ldots, p_{n} ; \lambda^{2}\right)\left\{\delta _ { i j } \left[\mu^{2} \frac{\partial}{\partial \mu^{2}}+\widetilde{B}_{\alpha} \alpha \frac{\partial}{\partial \alpha}+\widetilde{t}_{11} \gamma \frac{\partial}{\partial \gamma}\right.\right. \\
\left.\left.-\left[\mu^{2}\left(1-\widetilde{\Theta}_{1}\right)-\lambda^{2} \widetilde{\Theta}_{2}\right] \frac{\partial}{\partial \lambda^{2}}+\lambda^{2} \gamma \widetilde{\Omega} \frac{\partial}{\partial \kappa^{2}}\right]-\widetilde{T}_{i j}^{*}\right\} G_{j}^{\prime}
\end{gathered}
$$




$$
\begin{aligned}
& =-\left[\left(\Delta B_{\alpha}\right) \alpha \frac{\partial}{\partial \alpha}+\left(\Delta \Theta_{1}\right) \mu^{2} \frac{\partial}{\partial \lambda^{2}}+\left(\Delta \Theta_{2}\right) \lambda^{2} \frac{\partial}{\partial \lambda^{2}}\right. \\
& \left.+\Theta_{3} \kappa^{2} \frac{\partial}{\partial \lambda^{2}}+\frac{n}{2} \Delta \gamma_{\phi}\right] \widetilde{V}_{n}\left(p_{1}, \ldots, p_{n} ; \lambda^{2}\right) .
\end{aligned}
$$

From eq. (115), we will now extract a closed set of Callan-Symanzik equations satisfied by $D_{1}, \bar{D}, \bar{D}_{1}, D_{2}, \bar{D}_{2}, \ldots, D_{6}$, while expressing $\Delta B_{\gamma}, \Delta \Theta_{1}, \Delta \Theta_{2}, \Delta \gamma_{\phi}, \Theta_{3}$ in terms of $D_{1}, \bar{D}_{1}, D_{2}, D_{3}$. Let us first look at eq. (115) for the two-point function $(n=2)$ at $p^{2}=0$. From order- $\left(\lambda^{2}\right)^{0}$ terms, we obtain

$$
\Delta \Theta_{1}+\Delta \gamma_{\phi}=0
$$

which should be expected from eqs. (52a) and (66a). For order- $\lambda^{2}$ terms in eq.

(115), only the local vertex $\widetilde{O}_{1}^{\prime}$ and the effective coupling $D_{1}$ contribute to the lefthand side, yielding the equation

$$
-2 \mu^{2}\left(1-\widetilde{\Theta}_{1}\right) D_{1}=i \Delta \Theta_{2}+i \Delta \gamma_{\phi}-\left(\Delta \Theta_{1}\right) \mu^{2}\left[\frac{\partial^{2}}{\partial\left(\lambda^{2}\right)^{2}} \tilde{V}_{2}\left(p,-p ; \lambda^{2}\right)\right]_{p_{i}=0, \lambda^{2}=0}
$$

From order- $\kappa^{2}$ terms, we obtain

$$
-\mu^{2}\left(1-\widetilde{\Theta}_{1}\right) \bar{D}_{1}=i \Theta_{3} \text {. }
$$

Similarly, from the terms of orders $\left(\lambda^{2}\right)^{2}, \lambda^{2} \kappa^{2}$, and $\left(\kappa^{2}\right)^{2}$ respectively, we obtain the equations

$$
\begin{aligned}
& \mathrm{O}\left(\left(\lambda^{2}\right)^{2}\right): \\
& \left(\mu^{2} \frac{\partial}{\partial \mu^{2}}+\widetilde{B}_{\alpha} \alpha \frac{\partial}{\partial \alpha}+\widetilde{t}_{11} \gamma \frac{\partial}{\partial \gamma}+2 \widetilde{\Theta}_{2}\right) D_{1}+\gamma \widetilde{\Omega}_{1}-\sum_{j=1}^{6} \tilde{t}_{1 j}^{*} D_{j} \\
& \quad-2 \mu^{2}\left(1-\widetilde{\Theta}_{1}\right)\left[\frac{\partial}{\partial \lambda^{2}} \widetilde{V}_{2}\left(\widetilde{O}_{1}^{\prime}, p,-p ; \lambda^{2}\right)\right]_{p^{2}=\lambda^{2}=0} D_{1} \\
& \quad-\frac{1}{2} \mu^{2}\left(1-\widetilde{\Theta}_{1}\right) \sum_{i=2,3}\left[\frac{\partial^{2}}{\partial\left(\lambda^{2}\right)^{2}} \widetilde{V}_{2}\left(\widetilde{O}_{i}, p,-p ; \lambda^{2}\right)\right]_{p^{2}=\lambda^{2}=0} D_{i} \\
& \quad=-\left.\left[\left(\Delta B_{\alpha}\right) \alpha \frac{\partial}{\partial \alpha}+2 \Theta_{2}+\Delta \gamma_{\phi}\right] \frac{1}{2}\left[\frac{\partial^{2}}{\partial\left(\lambda^{2}\right)^{2}} \widetilde{V}_{2}\left(p,-p ; \lambda^{2}\right)\right]_{p}^{2}\right|_{\lambda^{2}=0} \\
& \quad-\left(\Delta \Theta_{1}\right) \mu^{2} \frac{1}{2}\left[\frac{\partial^{3}}{\partial\left(\lambda^{2}\right)^{3}} \widetilde{V}_{2}\left(p,-p ; \lambda^{2}\right)\right]_{p^{2}=\lambda^{2}=0}^{\mid}
\end{aligned}
$$




$$
\begin{aligned}
& \mathrm{O}\left(\lambda^{2} \kappa^{2}\right): \\
& \left(\mu^{2} \frac{\partial}{\partial \mu^{2}}+\widetilde{B}_{\alpha} \alpha \frac{\partial}{\partial \alpha}+\gamma \widetilde{t}_{11} \frac{\partial}{\partial \gamma}+\widetilde{\Theta}_{2}\right) \bar{D}_{1}+2 \gamma \widetilde{\Omega}_{\bar{D}}-\sum_{j=1} \tilde{t}_{1 j}^{*} \bar{D}_{j} \\
& \quad-\mu^{2}\left(1-\tilde{\Theta}_{1}\right)\left[\frac{\partial}{\partial \lambda^{2}} \tilde{V}_{2}\left(\tilde{O}_{1}^{\prime}, p,-p ; \lambda^{2}\right)\right]_{p^{2}=\lambda^{2}=0} \bar{D}_{1} \\
& \quad=-\Theta_{3}\left[\frac{\partial^{2}}{\partial\left(\lambda^{2}\right)^{2}} \tilde{V}_{2}\left(p,-p ; \lambda^{2}\right)\right]_{p^{2}=\lambda^{2}=0} . \\
& \mathrm{O}\left(\kappa^{4}\right): \\
& \left(\mu^{2} \frac{\partial}{\partial \mu^{2}}+\widetilde{B}_{\alpha} \alpha \frac{\partial}{\partial \alpha}+\gamma \tilde{t}_{11} \frac{\partial}{\partial \gamma}\right) \overline{\bar{D}}_{1}-\tilde{t}_{11} \overline{\bar{D}}_{1}=0
\end{aligned}
$$

where we have denoted $\widetilde{t}_{i j}^{*}=\tilde{t}_{j i}$.

In eq. (115), we may also look at the order- $p^{2}$ and order- $\left(p^{2}\right)^{2}$ terms for the $n=2$ case as well as the four- and six-point functions around the zero external momenta. From these considerations, one can easily obtain the following set of differential equations:

$$
\begin{aligned}
& \text { (i) } n=2, \mathrm{O}\left(p^{2}\right) \\
& \mathrm{O}\left(\left(\lambda^{2}\right)^{0}\right): \\
& \quad-\left(1-\widetilde{\Theta}_{1}\right) \mu^{2} D_{2}=-\left(\Delta \Theta_{1}\right) \mu^{2}\left[\frac{\partial}{\partial \lambda^{2}} \frac{\partial}{\partial p^{2}} \widetilde{V}_{2}\left(p,-p ; \lambda^{2}\right)\right]_{p^{2}=\lambda^{2}=0}-i \Delta \gamma_{\phi} .
\end{aligned}
$$

$$
\begin{aligned}
& \mathrm{O}\left(\lambda^{2}\right): \\
& \left(\mu^{2} \frac{\partial}{\partial \mu^{2}}+\widetilde{B}_{\alpha} \alpha \frac{\partial}{\partial \alpha}+\tilde{t}_{11} \gamma \frac{\partial}{\partial \gamma}+\widetilde{\Theta}_{2}\right) D_{2}+\gamma \widetilde{\Omega} D_{2}-\sum_{j=2}^{6} t_{2 j}^{*} D_{j} \\
& \quad-\left(1-\widetilde{\Theta}_{1}\right) \mu^{2} \sum_{i=2,3}\left[\frac{\partial^{2}}{\partial \lambda^{2} \partial p^{2}} \widetilde{V}_{2}\left(\widetilde{O}_{i}^{\prime}, p,-p ; \lambda^{2}\right)\right]_{p^{2}=\lambda^{2}=0} D_{i} \\
& \quad-2 \mu^{2}\left(1-\widetilde{\Theta}_{1}\right)\left[\frac{\partial}{\partial p^{2}} \tilde{V}\left(\tilde{O}_{1}^{\prime}, p,-p ; \lambda^{2}\right)\right]_{p^{2}=\lambda^{2}=0} D_{1} D_{p^{2}=\lambda^{2}=0}^{\mid} \\
& \quad-\left[\left(\Delta B_{\alpha}\right) \alpha \frac{\partial}{\partial \alpha}+\Delta \Theta_{2}+\Delta \gamma_{\phi}\right]\left[\frac{\partial^{2}}{\partial \lambda^{2} \partial p^{2}} \tilde{V}_{2}\left(p,-p ; \lambda^{2}\right)\right]_{p^{2}=\lambda^{2}=0} .
\end{aligned}
$$




$$
\begin{aligned}
& \mathrm{O}\left(\kappa^{2}\right) \\
& \left(\mu^{2} \frac{\partial}{\partial \mu^{2}}+\widetilde{B}_{\alpha} \alpha \frac{\partial}{\partial \alpha}+\widetilde{t}_{11} \gamma \frac{\partial}{\partial \gamma}\right) \bar{D}_{2}-\sum_{j=2,3} \tilde{t}_{2 j}^{*} \bar{D}_{j}=-\Theta_{3}\left[\frac{\partial^{2}}{\partial \lambda^{2} \partial p^{2}} \widetilde{V}_{2}\left(p,-p ; \lambda^{2}\right)\right]_{p^{2}=\lambda^{2}=0} \\
& \text { (ii) } n=4, p_{i}=0 \\
& \mathrm{O}\left(\left(\lambda^{2}\right)^{0}\right) \text { : } \\
& -\left(1-\widetilde{\Theta}_{1}\right) \mu^{2} D_{3}=i\left(\Delta B_{\alpha}\right) \alpha+2 i\left(\Delta \gamma_{\phi}\right) \alpha-\left(\Delta \Theta_{1}\right) \mu^{2}\left[\frac{\partial}{\partial \lambda^{2}} \widetilde{V}_{4}\left(p_{1}, \ldots, p_{4} ; \lambda^{2}\right)\right]_{p_{i}=0, \lambda^{2}=0} \\
& \mathrm{O}\left(\lambda^{2}\right) \\
& \left(\mu^{2} \frac{\partial}{\partial \mu^{2}}+\widetilde{B}_{\alpha} \alpha \frac{\partial}{\partial \alpha}+\widetilde{t}_{11} \gamma \frac{\partial}{\partial \gamma}+\Theta_{2}\right) D_{3}+\gamma \widetilde{\Omega} \bar{D}_{3}-\sum_{j=2}^{6} \tilde{t}_{3 j}^{*} D_{j} \\
& -\left(1-\widetilde{\Theta}_{1}\right) \mu^{2} \sum_{i=2,3}\left[\frac{\partial}{\partial \lambda^{2}} \tilde{V}_{4}\left(\tilde{O}_{i}^{\prime}, p_{1}, \ldots, p_{4} ; \lambda^{2}\right)\right]_{p_{i}=0, \lambda^{2}=0} D_{i} \\
& -\left.2\left(1-\widetilde{\Theta}_{1}\right) \mu^{2}\left[\widetilde{V}_{4}\left(\widetilde{O}_{1}^{\prime}, p_{1}, \ldots, p_{4} ; \lambda^{2}\right)\right]\right|_{p_{i}=0, \lambda^{2}=0} D_{1} \\
& =-\left[\left(\Delta B_{\alpha}\right) \alpha \frac{\partial}{\partial \alpha}+\Delta \Theta_{2}+2 \Delta \gamma_{\phi}\right]\left[\frac{\partial}{\partial \lambda^{2}} \widetilde{V}_{4}\left(p_{1}, \ldots, p_{4} ; \lambda^{2}\right)\right]_{p_{i}=0, \lambda^{2}=0} \\
& -\left(\Delta \Theta_{1}\right) \mu^{2}\left[\frac{\partial^{2}}{\partial\left(\lambda^{2}\right)^{2}} \tilde{V}_{4}\left(p_{1}, \ldots, p_{4} ; \lambda^{2}\right)\right]_{p_{i}=0, \lambda^{2}=0} .
\end{aligned}
$$

$\mathrm{O}\left(\kappa^{2}\right)$

$$
\begin{gathered}
\left(\mu^{2} \frac{\partial}{\partial \mu^{2}}+\widetilde{B}_{\alpha} \alpha \frac{\partial}{\partial \alpha}+\widetilde{t}_{11} \gamma \frac{\partial}{\partial \gamma}\right) \bar{D}_{3}-\sum_{j=2,3} \widetilde{t}_{3 j}^{*} \bar{D}_{j} \\
=-\Theta_{3}\left[\frac{\partial}{\partial \lambda^{2}} \widetilde{V}_{4}\left(p_{1}, \ldots, p_{4} ; \lambda^{2}\right)\right]_{p_{i}=0, \lambda^{2}=0} .
\end{gathered}
$$

(iii) $n=2, \mathrm{O}\left(\left(p^{2}\right)^{2}\right), \lambda^{2}=\kappa^{2}=0$

$$
\left(\mu^{2} \frac{\partial}{\partial \mu^{2}}+\widetilde{B}_{\alpha} \alpha \frac{\partial}{\partial \alpha}+\widetilde{t}_{11} \gamma \frac{\partial}{\partial \gamma}\right) D_{4}-\sum_{j=4}^{6} \widetilde{t}_{4 j}^{*} D_{j}
$$




$$
\begin{aligned}
& -\left(1-\widetilde{\Theta}_{1}\right) \mu^{2} \sum_{i=2,3} \frac{1}{2}\left[\frac{\partial^{2}}{\partial\left(p^{2}\right)^{2}} \widetilde{V}_{2}\left(\widetilde{O}_{i}^{\prime}, p,-p ; \lambda^{2}\right)\right]_{p^{2}=\lambda^{2}=0} D_{i} \\
& =-\left[\left(\Delta B_{\alpha}\right) \alpha \frac{\partial}{\partial \alpha}+\Delta \gamma_{\phi}\right] \frac{1}{2}\left[\frac{\partial^{2}}{\partial\left(p^{2}\right)^{2}} \widetilde{V}_{2}\left(p,-p ; \lambda^{2}\right)\right]_{p^{2}=\lambda^{2}=\mathbf{0}} \\
& -\left(\Delta \Theta_{1}\right) \mu^{2} \frac{1}{2}\left[\frac{\partial}{\partial \lambda^{2}} \frac{\partial^{2}}{\partial\left(p^{2}\right)^{2}} \widetilde{V}_{2}\left(p,-p ; \lambda^{2}\right)\right]_{p^{2}=\lambda^{2}=0} \text {. } \\
& \left(\mu^{2} \frac{\partial}{\partial \mu^{2}}+\widetilde{B}_{\alpha} \alpha \frac{\partial}{\partial \alpha}+\tilde{t}_{11} \gamma \frac{\partial}{\partial \gamma}\right) D_{5}-\sum_{j=4}^{6} \tilde{t}_{5 j}^{*} D_{j} \\
& -\left(1-\widetilde{\Theta}_{1}\right) \mu^{2} \sum_{i=2,3}\left[\frac{\partial}{\partial p_{i}^{2}} \widetilde{V}_{4}\left(\widetilde{O}_{i}^{\prime}, p_{1}, \ldots, p_{4} ; \lambda^{2}\right)\right]_{p_{i}=0, \lambda^{2}=0} D_{i} \\
& =-\left[\left(\Delta B_{\alpha}\right) \alpha \frac{\partial}{\partial \alpha}+2 \Delta \gamma_{\phi}\right]\left[\frac{\partial}{\partial p_{1}^{2}} \widetilde{V}_{4}\left(p_{1}, \ldots, p_{4} ; \lambda^{2}\right)\right]_{p_{i}=0, \lambda^{2}=0} \\
& -\left(\Delta \Theta_{1}\right) \mu^{2}\left[\frac{\partial^{2}}{\partial \lambda^{2} \partial p_{1}^{2}} \widetilde{V}_{4}\left(p_{1}, \ldots, p_{4} ; \lambda^{2}\right)\right]_{p_{i}=0, \lambda^{2}=0} . \\
& \left(\mu^{2} \frac{\partial}{\partial \mu^{2}}+\widetilde{B}_{\alpha} \alpha \frac{\partial}{\partial \alpha}+\tilde{t}_{11} \gamma \frac{\partial}{\partial \gamma}\right) D_{6}-\sum_{j=4}^{6} \tilde{t}_{6 j}^{*} D_{j} \\
& -\left(1-\widetilde{\Theta}_{1}\right) \mu^{2} \sum_{i=2,3}\left[\tilde{V}_{6}\left(\tilde{O}_{i}^{\prime}, p_{1}, \ldots, p_{6} ; \lambda^{2}\right)\right]_{p_{i}=0, \lambda^{2}=0} D_{i} \\
& =-\left.\left[\left(\Delta B_{\alpha}\right) \alpha \frac{\partial}{\partial \alpha}+3 \Delta \gamma_{\phi}\right]\left[\tilde{V}_{6}\left(p_{1}, \ldots, p_{6} ; \lambda^{2}\right)\right]\right|_{p_{i}=0, \lambda^{2}=0} \\
& -\left(\Delta \Theta_{1}\right) \mu^{2}\left[\frac{\partial}{\partial \lambda^{2}} \widetilde{V}_{6}\left(p_{1}, \ldots, p_{6} ; \lambda^{2}\right)\right]_{p_{i}=\mathbf{0}, \lambda^{2}=0} .
\end{aligned}
$$

Let us now denote the Taylor series of $\tilde{V}_{n}\left(p_{1}, \ldots, p_{n} ; \lambda^{2}\right)(n=2,4,6)$ around $p_{i}=0, \lambda^{2}=0$ such that

$$
\tilde{V}_{2}\left(p,-p ; \lambda^{2}\right)=-i \mu^{2}-i \lambda^{2}+i p^{2}+i p^{2} \frac{\lambda^{2}}{\mu^{2}}\left(\frac{\tilde{\gamma}_{\phi}}{1+\widetilde{\gamma}_{\phi}}\right)
$$




$$
\begin{aligned}
& -\frac{1}{2} i\left(\lambda^{2}\right)^{2} \frac{1}{\mu^{2}}\left(\frac{\widetilde{\Theta}_{2}+\widetilde{\gamma}_{\phi}}{1+\widetilde{\gamma}_{\phi}}\right) \\
& +\frac{1}{2} i\left(p^{2}\right)^{2} \frac{\tilde{\eta}_{1}}{\mu^{2}}+\frac{1}{2} i p^{2}\left(\lambda^{2}\right)^{2} \frac{\widetilde{\eta}_{2}}{\left(\mu^{2}\right)^{2}}+\frac{1}{2} i\left(p^{2}\right)^{2} \lambda^{2} \frac{\tilde{\eta}_{3}}{\left(\mu^{2}\right)^{2}}+\frac{1}{6} i\left(\lambda^{2}\right)^{3} \frac{\tilde{\eta}_{4}}{\left(\mu^{2}\right)^{2}}+\ldots, \\
& \widetilde{V}_{4}\left(p_{1}, p_{2}, p_{3}, p_{4} ; \lambda^{2}=-i \alpha-i \frac{\lambda^{2}}{\mu^{2}} \alpha\left(\frac{\widetilde{B}_{\alpha}+2 \widetilde{\gamma}_{\phi}}{1+\widetilde{\gamma}_{\phi}}\right)+i\left(p_{1}^{2}+p_{2}^{2}+p_{3}^{2}+p_{4}^{2}\right) \frac{\alpha \widetilde{\xi}_{1}}{\mu^{2}}\right. \\
& +i\left(p_{1}^{2}+p_{2}^{2}+p_{3}^{2}+p_{4}^{2}\right) \lambda^{2} \frac{\alpha \tilde{\xi}_{2}}{\left(\mu^{2}\right)^{2}}+\frac{1}{2} i\left(\lambda^{2}\right)^{2} \frac{\alpha \tilde{\xi}_{3}}{\left(\mu^{2}\right)^{2}}+\ldots, \\
& \widetilde{V}_{6}\left(p_{1}, \ldots, p_{6} ; \lambda^{2}\right)=i \frac{\widetilde{\delta}_{1}}{\mu^{2}}+i \lambda^{2} \frac{\widetilde{\delta}_{2}}{\left(\mu^{2}\right)^{2}}+\ldots,
\end{aligned}
$$

where we have defined the new constants $\widetilde{\eta}_{1}, \widetilde{\eta}_{2}, \widetilde{\eta}_{3}, \widetilde{\eta}_{4}, \widetilde{\xi}_{1}, \widetilde{\xi}_{2}, \widetilde{\xi}_{3}, \widetilde{\delta}_{1}, \widetilde{\delta}_{2}$. For various derivatives of $\widetilde{V}_{n}\left(\widetilde{O}_{i}^{\prime}, p_{1}, \ldots, p_{n} ; \lambda^{2}\right)$ appearing in eqs. (119)-(130), we have already introduced the constants $\tilde{a}_{i j}$ through the Taylor series (101). We now note that eqs. (116)-(118), (122), (125) relate the changes in the Callan-Symanzik coefficients to the functions $D_{1}, \bar{D}_{1}, D_{2}, D_{3}$ according to

$$
\begin{aligned}
& \Delta \gamma_{\phi}=-\Delta \Theta_{1}=-i \mu^{2}\left(1+\tilde{\gamma}_{\phi}\right)^{2} D_{2}, \\
& \Delta \Theta_{2}=i \mu^{2}\left(1+\tilde{\gamma}_{\phi}\right)\left\{2 D_{1}+\left(1-\widetilde{\Theta}_{2}\right) D_{2}\right\}, \\
& \alpha\left(\Delta B_{\alpha}\right)=i \mu^{2}\left(1+\tilde{\gamma}_{\phi}\right)\left\{D_{3}+\alpha\left(2-\widetilde{B}_{\alpha}\right) D_{2}\right\}, \\
& \Theta_{3}=i \mu^{2}\left(1+\widetilde{\gamma}_{\phi}\right) \bar{D}_{1} .
\end{aligned}
$$

(Acute readers might have noticed that eqs. (134)--(137) could have been directly obtained from eqs. $(66 \mathrm{c}-\mathrm{e}),(66 \mathrm{i})$ and the factorization (79).) Inserting the expressions (134)-(137) for $\Delta \gamma_{\phi}, \Delta \Theta_{1}, \Delta \Theta_{2}, \Delta B_{\alpha}, \Theta_{3}$ in the differential equations (119)-(121), (123), (124), (126)-(130), we finally obtain the Callan-Symanzik equations of the following forms

$$
\begin{aligned}
& \left(\mu^{2} \frac{\partial}{\partial \mu^{2}}+\widetilde{B}_{\alpha} \alpha \frac{\partial}{\partial \alpha}+\widetilde{t}_{11} \gamma \frac{\partial}{\partial \gamma}\right) D_{i}-\tilde{y}_{i j} D_{j}+\gamma \widetilde{\Omega} \bar{D}_{i}=0 \\
& \left(\mu^{2} \frac{\partial}{\partial \mu^{2}}+\widetilde{B}_{\alpha} \alpha \frac{\partial}{\partial \alpha}+\tilde{t}_{11} \gamma \frac{\partial}{\partial \gamma}\right) \bar{D}_{i}-\tilde{w}_{i j} \bar{D}_{j}+2 \gamma \widetilde{\Omega} \overline{\bar{D}}_{i}=0 \\
& \left(\mu^{2} \frac{\partial}{\partial \mu^{2}}+\tilde{B}_{\alpha} \alpha \frac{\partial}{\partial \alpha}+\widetilde{t}_{11} \gamma \frac{\partial}{\partial \gamma}\right) \overline{\bar{D}}_{1}-\widetilde{t}_{11} \overline{\bar{D}}_{1}=0, \\
& \bar{D}_{4}=\bar{D}_{5}=\bar{D}_{6}=\overline{\bar{D}}_{2}=\overline{\bar{D}}_{3}=\overline{\bar{D}}_{4}=\overline{\bar{D}}_{5}=\overline{\bar{D}}_{6}=0,
\end{aligned}
$$




$$
\begin{aligned}
& \tilde{y}_{i j}=\widetilde{I}_{i j}^{*}+\widetilde{S}_{i j}, \quad\left(\widetilde{S}_{i 4}=\widetilde{S}_{i 5}=\widetilde{S}_{i 6}=0 \text { for all } i\right), \\
& \tilde{w}_{i j}=\tilde{t}_{i j}^{*}+v_{i} \delta_{j 1},
\end{aligned}
$$

where non-vanishing $\widetilde{S}_{i j}$ are given by $\left(\tilde{a}_{i j}=\tilde{a}_{j i}^{*}\right.$ are defined in eq. (101))

$$
\begin{aligned}
& \tilde{S}_{11}=-4 \widetilde{\Theta}_{2}+2 \tilde{t}_{11} \text {, } \\
& \widetilde{S}_{12}=\frac{1}{2} \widetilde{t}_{12}^{*}+\left(1+\widetilde{\gamma}_{\phi}\right)\left\{-\frac{1}{2} \alpha\left(2-\widetilde{B}_{\alpha}\right) \frac{\partial}{\partial \alpha}\left(\frac{\widetilde{\Theta}_{2}+\widetilde{\gamma}_{\phi}}{1+\widetilde{\gamma}_{\phi}}\right)-\left(1-\widetilde{\Theta}_{2}\right) \frac{\widetilde{\Theta}_{2}+\widetilde{\gamma}_{\phi}}{1+\widetilde{\gamma}_{\phi}}\right. \\
& \left.+\frac{1}{2}\left(\widetilde{\Theta}_{2}+\widetilde{\gamma}_{\phi}\right)+\frac{1}{2} \widetilde{\eta}_{4}\left(1+\widetilde{\gamma}_{\phi}\right)\right\} \\
& \tilde{S}_{13}=\frac{1}{2} \widetilde{t}_{13}^{*}-\left(1+\tilde{\gamma}_{\phi}\right) \frac{1}{2} \frac{\partial}{\partial \alpha}\left(\frac{\widetilde{\Theta}_{2}+\widetilde{\gamma}_{\phi}}{1+\tilde{\gamma}_{\phi}}\right) \text {, } \\
& \widetilde{S}_{21}=2 \widetilde{\gamma}_{\phi}+2 \tilde{a}_{21}^{*} \\
& \tilde{S}_{22}=\tilde{\gamma}_{\phi}+\tilde{t}_{22}^{*}-\widetilde{\Theta}_{2}+\left(1+\tilde{\gamma}_{\phi}\right) \alpha\left(2-\widetilde{B}_{\alpha}\right) \frac{\partial}{\partial \alpha}\left(\frac{\tilde{\gamma}_{\phi}}{1+\widetilde{\gamma}_{\phi}}\right)-\widetilde{\Theta}_{2} \tilde{\gamma}_{\phi}-\tilde{\gamma}_{\phi}^{2} \\
& +\left(1+\tilde{\gamma}_{\phi}\right)^{2} \tilde{\eta}_{2} \\
& \widetilde{S}_{23}=\widetilde{t}_{23}^{*}+\left(1+\tilde{\gamma}_{\phi}\right) \frac{\partial}{\partial \alpha}\left(\frac{\tilde{\gamma}_{\phi}}{1+\widetilde{\gamma}_{\phi}}\right) \text {, } \\
& \widetilde{S}_{31}=2 \tilde{a}_{31}^{*}-2 \alpha\left(\tilde{B}_{\alpha}+2 \tilde{\gamma}_{\phi}\right), \\
& \widetilde{S}_{32}=\tilde{t}_{32}^{*}-\left(1+\tilde{\gamma}_{\phi}\right) \alpha\left(2-\widetilde{B}_{\alpha}\right) \frac{\partial}{\partial \alpha}\left[\frac{\alpha\left(\widetilde{B}_{\alpha}+2 \tilde{\gamma}_{\phi}\right)}{1+\tilde{\gamma}_{\phi}}\right]-\left(1-\widetilde{\Theta}_{2}\right) \alpha\left(\widetilde{B}_{\alpha}+2 \tilde{\gamma}_{\phi}\right) \\
& +2\left(1+\tilde{\gamma}_{\phi}\right) \alpha\left(\tilde{B}_{\alpha}+2 \tilde{\gamma}_{\phi}\right)+\left(1+\tilde{\gamma}_{\phi}\right)^{2} \alpha \tilde{\xi}_{3}, \\
& \tilde{S}_{33}=2 \tilde{\gamma}_{\phi}+\tilde{t}_{33}^{*}-\widetilde{\Theta}_{2}-\left(1+\tilde{\gamma}_{\phi}\right) \frac{\partial}{\partial \alpha}\left[\frac{\alpha\left(\tilde{B}_{\alpha}+2 \tilde{\gamma}_{\phi}\right)}{1+\tilde{\gamma}_{\phi}}\right] \text {, } \\
& \widetilde{S}_{41}=\widetilde{S}_{51}=\widetilde{S}_{61}=0, \\
& \tilde{S}_{42}=\frac{1}{2} \tilde{a}_{42}^{*}+\alpha\left(1+\tilde{\gamma}_{\phi}\right)\left(2-\widetilde{B}_{\alpha}\right) \frac{\partial \tilde{\eta}_{1}}{\partial \alpha}-\left(1+\tilde{\gamma}_{\phi}\right)^{2}\left(\widetilde{\eta}_{1}-\tilde{\eta}_{3}\right), \\
& \widetilde{S}_{43}=\frac{1}{2} \widetilde{a}_{43}^{*}+\left(1+\tilde{\gamma}_{\phi}\right) \frac{\partial \tilde{\eta}_{1}}{\partial \alpha} \\
& \widetilde{S}_{52}=a_{52}^{*}+\left(1+\widetilde{\gamma}_{\phi}\right) \alpha\left(2-\widetilde{B}_{\alpha}\right) \frac{\partial\left(\alpha \tilde{\xi}_{1}\right)}{\partial \alpha}-\left(1+\tilde{\gamma}_{\phi}\right)^{2} \alpha\left(2 \widetilde{\xi}_{1}-\widetilde{\xi}_{2}\right),
\end{aligned}
$$




$$
\begin{aligned}
& \tilde{S}_{53}=a_{53}^{*}+\left(1+\tilde{\gamma}_{\phi}\right) \frac{\partial}{\partial \alpha}\left(\alpha \tilde{\xi}_{1}\right), \\
& \tilde{S}_{62}=a_{62}^{*}+\left(1+\tilde{\gamma}_{\phi}\right) \alpha\left(2-\widetilde{B}_{\alpha}\right) \frac{\partial \tilde{\delta}_{1}}{\partial \alpha}-\left(1+\tilde{\gamma}_{\phi}\right)^{2}\left(3 \widetilde{\delta}_{1}-\widetilde{\delta}_{2}\right), \\
& \tilde{S}_{63}=a_{63}^{*}+\left(1+\tilde{\gamma}_{\phi}\right) \frac{\partial}{\partial \alpha} \widetilde{\delta}_{1},
\end{aligned}
$$

and the constants $v_{i}(i=1,2,3)$, defined in eq. (143), are given by

$$
\begin{aligned}
& v_{1}=\tilde{\gamma}_{\phi}+\tilde{t}_{11}-\tilde{\Theta}_{2}-\tilde{\gamma}_{\phi}=\tilde{t}_{11}-\widetilde{\Theta}_{2}, \\
& v_{2}=\tilde{\gamma}_{\phi}, \\
& v_{3}=-\alpha\left(\tilde{B}_{\alpha}+2 \tilde{\gamma}_{\phi}\right) .
\end{aligned}
$$

Since the effective couplings $D_{i}(i=1, \ldots, 6), \bar{D}_{i}(i=1,2,3), \overline{\bar{D}}_{1}$ are dimensionless, the Callan-Symanzik equations (138), (139), (140) may be written as $\left(\bar{D}_{4}=\bar{D}_{5}=\right.$ $\bar{D}_{6}=\overline{\bar{D}}_{2}=\overline{\bar{D}}_{3}=\overline{\bar{D}}_{4}=\overline{\bar{D}}_{5}=\overline{\bar{D}}_{6}=0$ )

$$
\begin{aligned}
& \left(M^{2} \frac{\partial}{\partial M^{2}}-\widetilde{B}_{\alpha} \alpha \frac{\partial}{\partial \alpha}-\widetilde{t}_{11} \gamma \frac{\partial}{\partial \gamma}\right) D_{i}+\tilde{y}_{i j} D_{j}-\gamma \widetilde{\Omega} \bar{D}_{i}=0 \\
& \left(M^{2} \frac{\partial}{\partial M^{2}}-\widetilde{B}_{\alpha} \alpha \frac{\partial}{\partial \alpha}-\widetilde{t}_{11} \gamma \frac{\partial}{\partial \gamma}\right) \bar{D}_{i}+\widetilde{w}_{i j} \bar{D}_{j}-2 \gamma \widetilde{\Omega} \overline{\bar{D}}_{i}=0 \\
& \left(M^{2} \frac{\partial}{\partial M^{2}}-\widetilde{B}_{\alpha} \alpha \frac{\partial}{\partial \alpha}-\widetilde{t}_{11} \gamma \frac{\partial}{\partial \gamma}\right) \overline{\bar{D}}_{1}-\widetilde{t}_{11} \overline{\bar{D}}_{1}=0 .
\end{aligned}
$$

Evidently, eqs. (146)-(148) have the desired forms which can identify arbitrary powers of $\log \left(M^{2} / \mu^{2}\right)$ in a systematic fashion. These partial differential equations can be easily integrated by introducing effective coupling constants and appropriate anomalous dimensions in the usual way. As an illustration, in sect. 4, we will sum all the leading logarithms appearing in perturbative calculations of the effective couplings, by using eqs. (146)-(148).

Finally, we should mention that, among the effective couplings above, we are only interested in $D_{4}\left(=C_{1}\right), D_{5}\left(=C_{2}\right), D_{6}\left(=C_{3}\right)$. The additional couplings $D_{1}, \bar{D}_{1}, \overline{\bar{D}}_{1}$, $D_{2}, \bar{D}_{2}, D_{3}, \bar{D}_{3}$ here have a role of linearizing the Callan-Symanzik equations for $D_{4}, D_{5}, D_{6}$, as well as acounting for the changes in Callan-Symanzik coefficients due to the influence of virtual heavy particles.

\section{Explicit calculations}

In the foregoing two sections, we have established the factorization and derived the Callan-Symanzik equations satisfied by new effective couplings. In this section, we 
will perform lowest non-trivial-order calculations explicitly and then use the CallanSymanzik equations to sum all the leading logarithms. In this consideration, there appear two interesting physical limits

Case I: $\quad \alpha, \beta, \gamma<<1, \quad \gamma \sim \alpha, \quad \alpha \log M^{2} \sim \mathrm{O}(1), \quad \gamma \log M^{2} \sim \mathrm{O}(1)$,

Case II: $\quad \alpha, \beta, \gamma<<1, \quad \gamma<<\alpha, \quad \alpha \log M^{2} \sim \mathrm{O}(1), \quad \gamma \log M^{2}<<1$.

(For simplicity, we will assume $\beta<<\gamma$ for both cases). We will consider these two cases below.

Let us first calculate the effective couplings $D_{i}(i=1,2, \ldots, 6), \bar{D}_{i}(i=1,2,3)$, $\bar{D}_{1}$ in the lowest non-trivial order. The factorization (79) and the normalization conditions $(75 a-p)$ for various local vertices imply that, for $M^{2}>>\mu^{2}, \lambda^{2}, \kappa^{2}$,

$$
\begin{aligned}
& V_{2,0}\left(p,-p ; \lambda^{2}, \kappa^{2}\right)-\widetilde{V}_{2}\left(p,-p ; \lambda^{2}\right)_{p^{2}, \lambda^{2}, \kappa^{2} \rightarrow 0} ; \frac{1}{M^{2}}\left[\left(\lambda^{2}\right)^{2} D_{1}+\lambda^{2} \kappa^{2} \bar{D}_{1}\right. \\
& \left.+\left(\kappa^{2}\right)^{2} \bar{D}_{1}+\lambda^{2} p^{2} D_{2}+\kappa^{2} p^{2} \bar{D}_{2}+\left(p^{2}\right)^{2} D_{4}\right]+\mathrm{O}\left(\frac{1}{M^{4}}\right) \\
& V_{4,0}\left(p_{1}, p_{2}, p_{3}, p_{4} ; \lambda^{2}, \kappa^{2}\right)-\widetilde{V}_{4}\left(p_{1}, p_{2}, p_{3}, p_{4} ; \lambda^{2}\right)_{p_{i}, \lambda, \kappa \rightarrow 0}^{=} \frac{1}{M^{2}}\left[\lambda^{2} D_{3}\right. \\
& \left.\quad+\kappa^{2} \bar{D}_{3}+\left(p_{1}^{2}+p_{2}^{2}+p_{3}^{2}+p_{4}^{2}\right) D_{5}\right]+\mathrm{O}\left(\frac{1}{M^{4}}\right), \\
& V_{6,0}\left(p_{1}, \ldots, p_{6} ; \lambda^{2}, \kappa^{2}\right)-\tilde{V}_{6}\left(p_{1}, \ldots, p_{6} ; \lambda^{2}\right)_{p_{i}, \lambda, \kappa \rightarrow 0}^{=} \frac{1}{M^{2}} D_{6}+\mathrm{O}\left(\frac{1}{M^{4}}\right) .(151)
\end{aligned}
$$

In fig. 8, we show the Feynman diagrams which contribute to the left-hand sides of eqs. (149)-(151) and are necessary for the lowest non-trivial-order evaluations of the effective couplings $D_{i}, \bar{D}_{i}, \bar{D}_{1}$. The lowest non-trivial order contributions to $D_{1}, \bar{D}_{1}, D_{2}, \bar{D}_{2}, D_{4}$ come from the Feynman diagrams (b), (c) in fig. $8, \bar{D}_{1}$ from the Feynman diagram (a), $\bar{D}_{3}$ and $D_{5}$ from the Feynman diagram (d), $D_{3}$ from the Feynman diagrams (e), (f), (g), (h), and $D_{6}$ from the Feynman diagrams (i), (g), (h), Once corresponding Feynman integrals for these diagrams are evaluated, it is a simple matter to read off $D_{1}, \bar{D}_{1}, \overline{\bar{D}}_{1}, D_{2}, \bar{D}_{2}, D_{3}, \bar{D}_{3}, D_{4}, D_{5}, D_{6}$ from the results via eqs. (149)-(151). Here, we give the results:

Case I $(\gamma \sim \alpha)$ :

$$
\begin{aligned}
& D_{1}=-\frac{i}{3 \cdot 2^{10} \pi^{4}} \gamma^{2}\left[\log \frac{M^{2}}{\mu^{2}}+\frac{7}{6}\right]+\mathrm{O}\left(\gamma^{2} \alpha, \gamma^{3}\right) \\
& \bar{D}_{1}=\frac{i}{2^{9} \pi^{4}} \gamma^{2}\left[\log \frac{M^{2}}{\mu^{2}}+1\right]+\mathrm{O}\left(\gamma^{2} \alpha, \gamma^{3}\right) \\
& \overline{\bar{D}}_{1}=-\frac{i}{64 \pi^{2}} \gamma+\mathrm{O}\left(\gamma^{2}, \gamma \alpha\right)
\end{aligned}
$$


C. Lee / Theory of virtual heavy particle effects

215

$$
\begin{aligned}
& D_{2}=-\frac{i}{3 \cdot 2^{10} \pi^{4}} \gamma^{2}\left[\log \frac{M^{2}}{\mu^{2}}+\frac{1}{6}\right]+\mathrm{O}\left(\gamma^{2} \alpha, \gamma^{3}\right) \\
& \bar{D}_{2}=-\frac{i}{2^{10} \pi^{4}} \gamma^{2}+\mathrm{O}\left(\gamma^{2} \alpha, \gamma^{3}\right) \\
& D_{3}=-\frac{i}{2^{8} \pi^{4}} \gamma^{2} \alpha\left[\log \frac{M^{2}}{\mu^{2}}+\frac{7}{6}\right]+\frac{3 i}{2^{8} \pi^{4}} \gamma^{3}\left[\log \frac{M^{2}}{\mu^{2}}+1\right]+\mathrm{O}\left(\gamma^{2} \alpha^{2}, \gamma^{3} \alpha, \gamma^{4}\right) \\
& \bar{D}_{3}=-\frac{3 i}{32 \pi^{2}} \gamma^{2}+\mathrm{O}\left(\gamma^{2} \alpha, \gamma^{3}\right) \\
& D_{4}=\frac{i}{9 \cdot 2^{11} \pi^{4}} \gamma^{2}+\mathrm{O}\left(\gamma^{2} \alpha, \gamma^{3}\right) \\
& D_{5}=\frac{i}{3 \cdot 2^{6} \pi^{2}} \gamma^{2}+\mathrm{O}\left(\gamma^{2} \alpha, \gamma^{3}\right) \\
& D_{6}=-\frac{15 i}{32 \pi^{2}} \gamma^{3}+\mathrm{O}\left(\gamma^{3} \alpha, \gamma^{4}, \gamma^{2} \alpha^{2}\right)
\end{aligned}
$$

Case II $(\gamma<<\alpha)$ :

$D_{1}, \bar{D}_{1}, \overline{\bar{D}}_{1}, D_{2}, \bar{D}_{2}, \bar{D}_{3}, D_{4}, D_{5}$ are same with the case I if one replaces $\mathrm{O}\left(\gamma^{2} \alpha, \gamma^{3}\right) \rightarrow \mathrm{O}\left(\gamma^{2} \alpha\right), \mathrm{O}\left(\gamma^{2}, \gamma \alpha\right) \rightarrow \mathrm{O}(\gamma \alpha)$, and

$$
\begin{aligned}
& D_{3}=-\frac{i}{2^{8} \pi^{4}} \gamma^{2} \alpha\left[\log \frac{M^{2}}{\mu^{2}}+\frac{7}{6}\right]+\mathrm{O}\left(\gamma^{2} \alpha^{2}\right), \\
& D_{6}=-\frac{15 i}{2^{9} \pi^{4}} \gamma^{2} \alpha^{2}\left[\log \frac{M^{2}}{\mu^{2}}+\frac{7}{6}\right]+\mathrm{O}\left(\gamma^{2} \alpha^{3}\right) .
\end{aligned}
$$

The next quantities which we calculate are the constants $\widetilde{B}_{\alpha}, \widetilde{\gamma}_{\phi}, \widetilde{\Theta}_{1}, \widetilde{\Theta}_{2}, \tilde{\eta}_{1}, \tilde{\eta}_{2}$, $\tilde{\eta}_{3}, \tilde{\eta}_{4}, \widetilde{\xi}_{1}, \widetilde{\xi}_{2}, \widetilde{\xi}_{3}, \widetilde{\delta}_{1}, \widetilde{\delta}_{2}$. As defined in eqs. (131)-(133), these constants can be read off from appropriate coefficients of the Taylor series for $V_{n}\left(p_{1}, \ldots, p_{n} ; \lambda^{2}\right)$ $(n=2,4,6)$ around $p_{i}=0$ and $\lambda^{2}=0$. We show the Feynman diagrams necessary for the calculation of the lowest non-trivial-order contributions to $V_{n}\left(p_{1}, \ldots, p_{n} ; \lambda^{2}\right)$ $(n=2,4,6)$ in fig. 9 . After some calculations, we obtain the following values in the lowest non-trivial-order

$$
\begin{aligned}
& \tilde{\gamma}_{\phi}=-\frac{\alpha^{2}}{3 \cdot 2^{10} \pi^{4}}, \quad \tilde{\eta}_{2}=\frac{\alpha^{2}}{3 \cdot 2^{10} \pi^{4}}, \quad \tilde{\Theta}_{2}=\frac{\alpha}{32 \pi^{2}}, \quad \tilde{\eta}_{4}=\frac{\alpha}{32 \pi^{2}}, \\
& \tilde{\eta}_{1}=\frac{\alpha^{2} A}{3 \cdot 2^{8} \pi^{4}}, \quad \tilde{\eta}_{3}=-\frac{\alpha^{2} A}{3 \cdot 2^{8} \pi^{4}}, \quad \widetilde{B}_{\alpha}=\frac{3 \alpha}{32 \pi^{2}}, \quad \tilde{\xi}_{1}=\frac{\alpha}{3 \cdot 2^{6} \pi^{2}},
\end{aligned}
$$




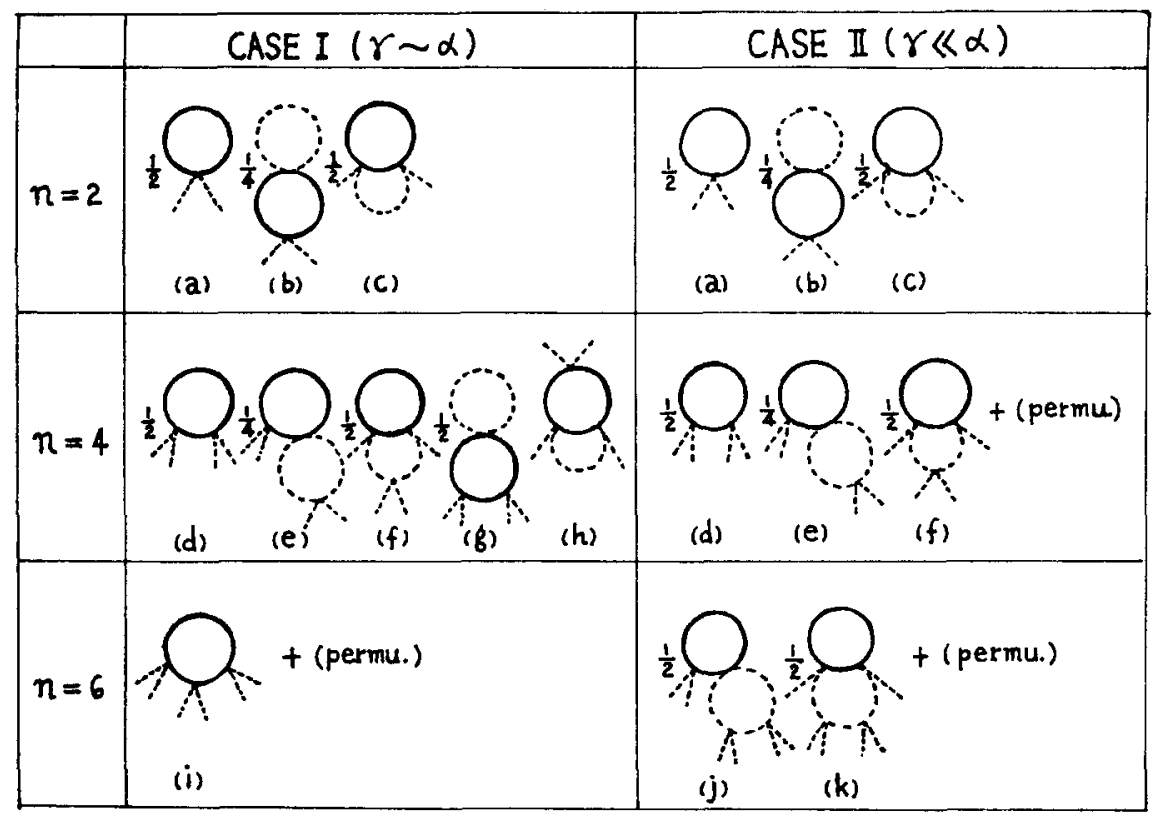

Fig. 8. Feynman diagrams necessary to evaluate the effective coupling in the lowest non-trivial order.

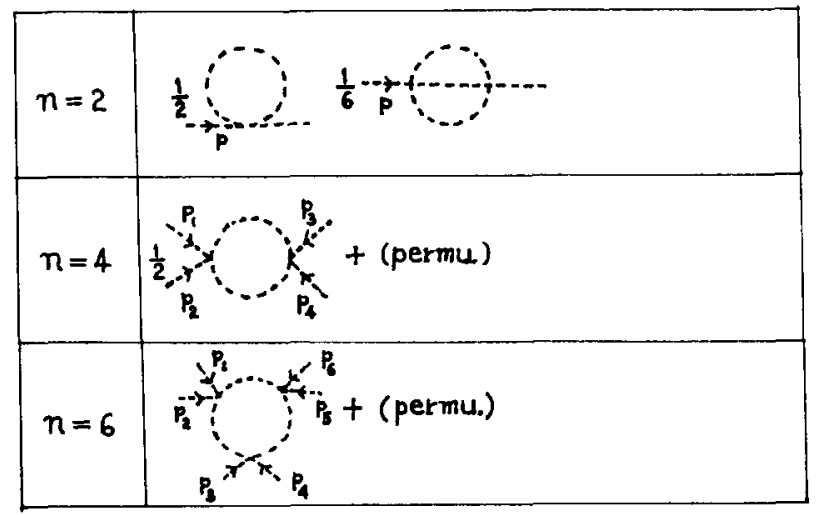

Fig. 9. Feynman diagrams necessary to evaluate the Callan-Symanzik coefficients in the lowest non-trivial order. 


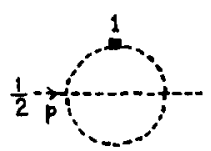

$\left(\tilde{a}_{12}\right)$

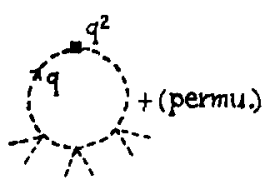

$\left(\tilde{a}_{26}\right)$<smiles>CCC1(C)CCC(C)(C)CC1</smiles>

$\left(\tilde{a}_{13}\right)$

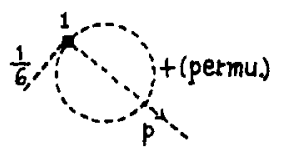

$\left(\tilde{a}_{34}\right)$

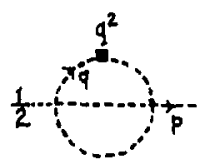

$\left(\tilde{a}_{24}\right)$<smiles>[Y][C@H]1CCC(C)(C)[C@@H](C)C1</smiles>

$\left(\tilde{a}_{35}\right)$<smiles>C=CC1(C)[C@H](C)CCC(C)(C)[C@H]1C</smiles>

$\left(\tilde{Q}_{25}\right)$

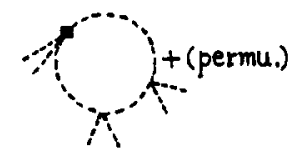

$\left(\tilde{a}_{36}\right)$

Fig. 10. Feynman diagrams necessary to evaluate $\tilde{a}_{i j}$ in the lowest non-trivial order.

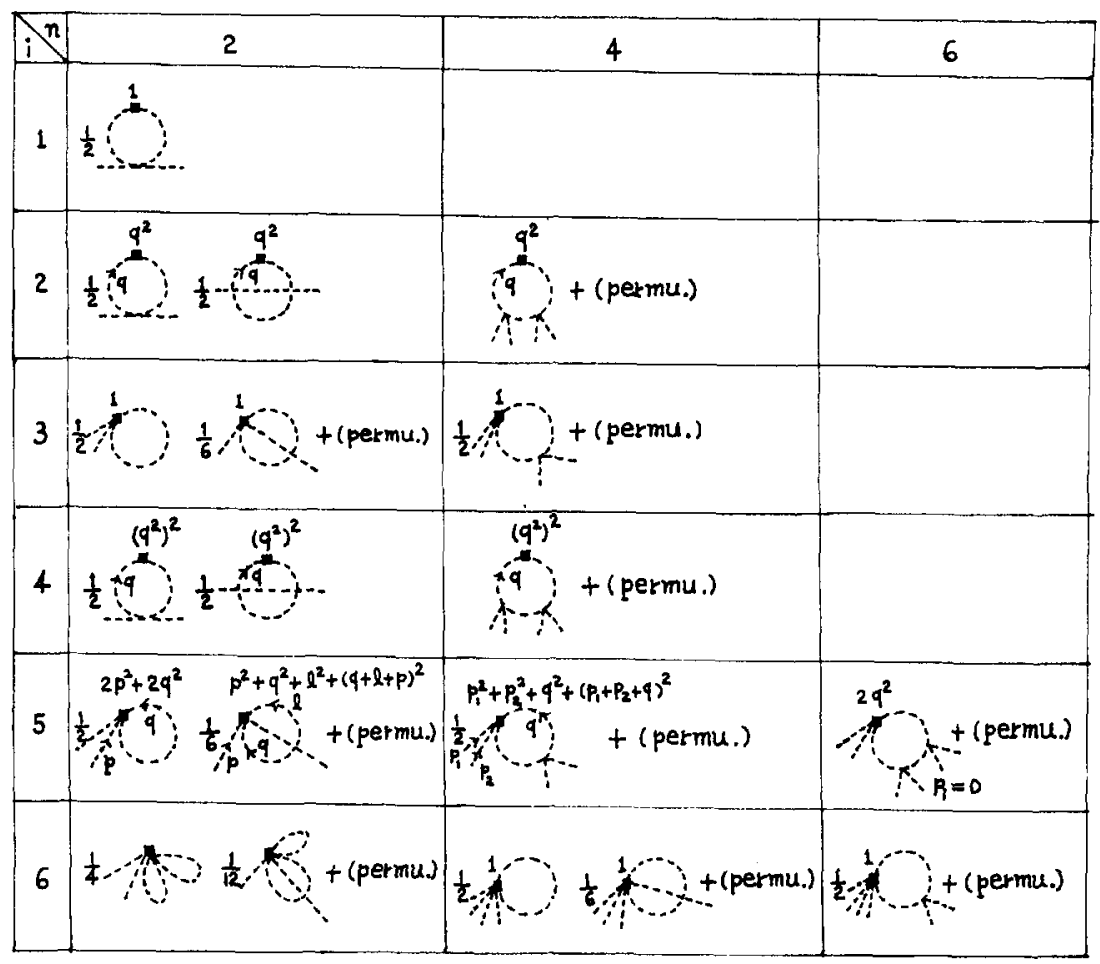

Fig. 11. Feynman diagtams necessary to evaluate $\tilde{t}_{i j}$ in the lowest non-trivial order. Here, we have set $\rho_{\phi}=\rho_{\psi}=1$. 


$$
\tilde{\xi}_{2}=\frac{-\alpha}{3 \cdot 2^{6} \pi^{2}}, \quad \tilde{\xi}_{3}=\frac{3 \alpha}{32 \pi^{2}}, \quad \delta_{1}=-\frac{15 \alpha^{3}}{32 \pi^{2}}, \quad \delta_{2}=\frac{15 \alpha^{3}}{32 \pi^{2}},
$$

where the pure number $A$ appearing in $\tilde{\eta}_{1}, \widetilde{\eta}_{3}$ is given by the integral

$$
\begin{aligned}
A & =\int_{0}^{1} \mathrm{~d} \kappa \int_{1}^{\infty} \mathrm{d} y \frac{(y-1)^{2}}{2 y^{4}} \frac{1}{\left[\left(\kappa-\kappa^{2}\right)(y-1)+1\right]} \\
& =32 \int_{1}^{\infty} \mathrm{d} x\left[\frac{1}{\left(x^{2}+3\right)^{3}}-\frac{4}{\left(x^{2}+3\right)^{4}}\right] \log \left(\frac{x+1}{x-1}\right) .
\end{aligned}
$$

Calculations of the constants $\tilde{a}_{12}, \tilde{a}_{13}, \tilde{a}_{24}, \tilde{a}_{25}, \tilde{a}_{26}, \tilde{a}_{34}, \tilde{a}_{35}, \tilde{a}_{36}$ defined in eq. (101) are quite analogous to the calculations of the constants given in eq. (154). Feynman diagrams necessary for these calculations are depicted in fig. 10 . In the lowest non-trivial order, we do not need to do any additional calculation, since the constants $\tilde{a}_{i j}$ can be related to the constants just given in eq. (154) as follows:

$$
\begin{aligned}
& \tilde{a}_{12}=-\tilde{\gamma}_{\phi}=\frac{\alpha^{2}}{3 \cdot 2^{10} \pi^{4}}, \\
& \tilde{a}_{13}=\alpha\left(\tilde{B}_{\alpha}+2 \tilde{\gamma}_{\phi}\right)=\frac{3 \alpha^{2}}{32 \pi^{2}}, \\
& \tilde{a}_{24}=-\left(3 \tilde{\eta}_{1}+\tilde{\eta}_{3}\right)\left(1+\tilde{\gamma}_{\phi}\right)=-\frac{2 \alpha^{2}}{3 \cdot 2^{8} \pi^{4}} A, \\
& \tilde{a}_{25}=-\left(2 \alpha \tilde{\xi}_{1}+\alpha \tilde{\xi}_{2}\right)=-\frac{\alpha^{2}}{3 \cdot 2^{6} \pi^{2}}, \\
& \tilde{a}_{26}=-15\left(\widetilde{\delta}_{1}+\widetilde{\delta}_{2}\right)\left(1+\tilde{\gamma}_{\phi}\right)=\mathrm{O}\left(\alpha^{4}\right), \\
& \tilde{a}_{34}=-2 \frac{\tilde{\eta}_{1}}{\alpha}\left(1+\tilde{\gamma}_{\phi}\right)=-\frac{2 \alpha}{3 \cdot 2^{8} \pi^{4}} A, \\
& \tilde{a}_{35}=-2 \tilde{\xi}_{1}\left(1+\tilde{\gamma}_{\phi}\right)=-\frac{2 \alpha}{3 \cdot 2^{6} \pi^{2}}, \\
& \tilde{a}_{36}=-15 \frac{\tilde{\delta}_{1}}{\alpha}\left(1+\tilde{\gamma}_{\phi}\right)=\frac{225 \alpha^{2}}{32 \pi^{2}} \cdot
\end{aligned}
$$

Elements of the anomalous dimension matrix $\tilde{t}_{i j}$ can be obtained from the Taylor series of $\widetilde{V}_{n}\left(\widetilde{O}_{i}^{\prime}, p_{1}, \ldots, p_{n} ; \lambda^{2}\right)(n=2,4,6)$ around $p_{i}=0, \lambda^{2}=0$, as indicated in eq. (101). Feynman diagrams necessary for the lowest non-trivial-order calculations of $\widetilde{V}_{n}\left(\widetilde{O}_{i}^{\prime}, p_{1}, \ldots, p_{n} ; \lambda^{2}\right)(n=2,4,6)$ are shown in fig. 11 . Again, we just summa- 
rize the result of calculations here:

$$
\left\{t_{i j}\right\}=\left[\begin{array}{llllll}
\frac{\alpha}{32 \pi^{2}} & 0 & 0 & 0 & 0 & 0 \\
\frac{\alpha}{16 \pi^{2}} & \mathrm{O}\left(\alpha^{2}\right) & \frac{3 \alpha^{2}}{16 \pi^{2}} & 0 & 0 & 0 \\
\frac{1}{32 \pi^{2}} & \frac{2 \alpha}{3 \cdot 2^{10} \pi^{4}} & \frac{3 \alpha}{32 \pi^{2}} & 0 & 0 & 0 \\
\mathrm{O}(\alpha) & \mathrm{O}\left(\alpha^{2}\right) & \mathrm{O}\left(\alpha^{2}\right) & \mathrm{O}\left(\alpha^{2}\right) & \mathrm{O}\left(\alpha^{2}\right) & \mathrm{O}\left(\alpha^{3}\right) \\
\frac{1}{16 \pi^{2}} & \frac{1}{16 \pi^{2}} & \frac{3 \alpha}{4 \pi^{2}} & \frac{2 \alpha}{9 \cdot 2^{9} \pi^{4}} & \frac{3 \alpha}{32 \pi^{2}} & \frac{45 \alpha^{2}}{8 \pi^{2}} \\
\mathrm{O}(\alpha) & \mathrm{O}(\alpha) & \frac{1}{32 \pi^{2}} & \mathrm{O}(\alpha) & \mathrm{O}(\alpha) & \frac{15 \alpha}{32 \pi^{2}}
\end{array}\right] .
$$

The Callan-Symanzik coefficient matrix $\left\{\tilde{y}_{i j}\right\}$ in eq. (146) can be found by adding the matrix $\left\{\widetilde{S}_{i j}\right\}$ to the transpose of the above anomalous dimension matrix. The constants $\tilde{S}_{i j}$ are obtained by inserting the expressions (154), (156), (157) into eq. (144). After some straightforward algebra, one obtains

$$
\left\{\widetilde{y}_{i j}\right\}=\left[\begin{array}{cccccc}
-\frac{\alpha}{32 \pi^{2}} & \frac{\alpha}{16 \pi^{2}} & \frac{1}{32 \pi^{2}} & O(\alpha) & \frac{1}{16 \pi^{2}} & O(\alpha) \\
O\left(\alpha^{3}\right) & -\frac{\alpha}{32 \pi^{2}} & \frac{\alpha}{3 \cdot 2^{9} \pi^{4}} & O\left(\alpha^{2}\right) & \frac{1}{16 \pi^{2}} & O(\alpha) \\
O\left(\alpha^{3}\right) & \frac{3 \alpha^{2}}{16 \pi^{2}} & -\frac{\alpha}{32 \pi^{2}} & O\left(\alpha^{2}\right) & \frac{3 \alpha}{4 \pi^{2}} & \frac{1}{32 \pi^{2}} \\
0 & \frac{\alpha^{2} A}{3 \cdot 2^{8} \pi^{4}} & \frac{\alpha A}{3 \cdot 2^{8} \pi^{4}} & O\left(\alpha^{2}\right) & \frac{\alpha}{9 \cdot 2^{8} \pi^{4}} & O(\alpha) \\
0 & O\left(\alpha^{3}\right) & O\left(\alpha^{2}\right) & O\left(\alpha^{2}\right) & \frac{3 \alpha}{32 \pi^{2}} & O(\alpha) \\
0 & -\frac{15 \alpha^{3}}{16 \pi^{2}} & \frac{45 \alpha^{2}}{8 \pi^{2}} & O\left(\alpha^{3}\right) & \frac{45 \alpha^{2}}{8 \pi^{2}} & \frac{15 \alpha}{32 \pi^{2}}
\end{array}\right] .
$$

The constants $v_{i}(i=1,2,3)$ (see eq. (145)) in the lowest non-trivial order is given by

$$
v_{1}=O\left(\alpha^{2}\right), \quad v_{2}=-\frac{\alpha^{2}}{3 \cdot 2^{10} \pi^{4}}, \quad v_{3}=-\frac{3 \alpha^{2}}{32 \pi^{2}}
$$


Finally, we give the lowest non-trivial-order value for the constant $\Omega$ defined in eq. (114),

$$
\Omega=\frac{1}{32 \pi^{2}}
$$

With these lowest non-trivial-order calculations at hand, let us see what the Callan-Symanzik equations (146)-(148) do for us. Let us first consider the case II (i.e., $\gamma<<\alpha$ ). In the case II, suppose we want to sum the series

$$
\begin{aligned}
& D_{1} \equiv \frac{\gamma^{2}}{\alpha} W_{1}, \quad D_{2} \equiv \frac{\gamma^{2}}{a} W_{2}, \quad D_{3} \equiv \gamma^{2} W_{3}, \\
& D_{4} \equiv \gamma^{2} W_{4}, \quad D_{5} \equiv \gamma^{2} W_{5}, \quad D_{6} \equiv \gamma^{2} \alpha W_{6}, \\
& W_{i}=\sum_{k=0}^{\infty} d_{k}^{(i)}\left(\alpha \log \frac{M^{2}}{\mu^{2}}\right)^{k}, \quad(i=1,2, \ldots, 6),
\end{aligned}
$$

with the boundary values (see eq. (153))

$$
\begin{aligned}
& d_{0}^{(1)}=d_{0}^{(2)}=d_{0}^{(3)}=d_{0}^{(6)}=0 \\
& d_{0}^{(4)}=\frac{i}{9 \cdot 2^{11} \pi^{4}}, \quad d_{0}^{(5)}=\frac{i}{3 \cdot 2^{6} \pi^{2}} .
\end{aligned}
$$

The first observation one may make in the Callan-Symanzik equations (146)-(148) is that one does not need to consider the functions $\bar{D}_{1}, \bar{D}_{1}, \bar{D}_{2}, \bar{D}_{3}$ for the series (161) since they contribute $\mathrm{O}\left(\gamma^{3}\right)$ terms at most. Secondly, from the known behavior of the Callan-Symanzik coefficients, one may observe that there will be no higher logarithms than the ones kept in the series (161); i.e., the series (161) correspond to the leading log sum. Moreover, in terms of the functions $W_{i}(i=1,2, \ldots, 6)$, the CallanSymanzik equation (146), with the functions $\bar{D}_{i}$ ignored, becomes

$$
\begin{aligned}
& i=1,2: \\
& \left(M^{2} \frac{\partial}{\partial M^{2}}-\widetilde{B}_{\alpha} \alpha \frac{\partial}{\partial \alpha}+\widetilde{B}_{\alpha}-2 \widetilde{t}_{11}\right) w_{i}+\sum_{j=1}^{2} \tilde{y}_{i j} w_{j}+\sum_{j=3}^{5} \alpha \tilde{y}_{i j} w_{j}+\alpha^{2} y_{i 6} w_{6}=0 \\
& i=3,4,5: \\
& \left(M^{2} \frac{\partial}{\partial M^{2}}-\widetilde{B}_{\alpha} \alpha \frac{\partial}{\partial \alpha}-2 \widetilde{t}_{11}\right) w_{i}+\sum_{j=1}^{2}\left(\frac{\tilde{y}_{i j}}{\alpha}\right) w_{j}+\sum_{j=3}^{5} \tilde{y}_{i j} w_{j}+\tilde{y}_{i 6} \alpha W_{6}=0
\end{aligned}
$$




$$
\begin{aligned}
& i=6: \\
& \left(M^{2} \frac{\partial}{\partial M^{2}}-\tilde{B}_{\alpha} \alpha \frac{\partial}{\partial \alpha}-\tilde{B}_{\alpha}-2 \tilde{t}_{11}\right) w_{6}+\sum_{j=1}^{2}\left(\frac{\tilde{y}_{6 j}}{\alpha^{2}}\right) w_{j}+\sum_{j=3}^{5}\left(\frac{\tilde{y}_{6 j}}{\alpha}\right) w_{j}+\tilde{y}_{66} w_{6}=0
\end{aligned}
$$

Inserting the lowest non-trivial-order results for $\tilde{y}_{i j}$ (see eq. (158), one can easily convince oneself that the leading log sum (161) can be completely determined from the partial differential equations

$$
\left(M^{2} \frac{\partial}{\partial M^{2}}-\frac{3 \alpha^{2}}{32 \pi^{4}} \frac{\partial}{\partial \alpha}\right) w_{i}+\alpha X_{i j} w_{j}=0, \quad(i, j=1, \ldots, 6),
$$

with the boundary values

$$
\begin{aligned}
& \left.\left(W_{0}\right)_{i} \equiv W_{i}\right|_{M^{2}=\mu^{2}}, \quad(i=1, \ldots, 6), \\
& \left(W_{0}\right)_{1}=\left(W_{0}\right)_{2}=\left(W_{0}\right)_{3}=\left(W_{0}\right)_{6}=0, \quad\left(W_{0}\right)_{4}=\frac{i}{9 \cdot 2^{11} \pi^{4}}, \quad\left(W_{0}\right)_{5}=\frac{i}{3 \cdot 2^{6} \pi^{2}},
\end{aligned}
$$

and the matrix $X$ given by

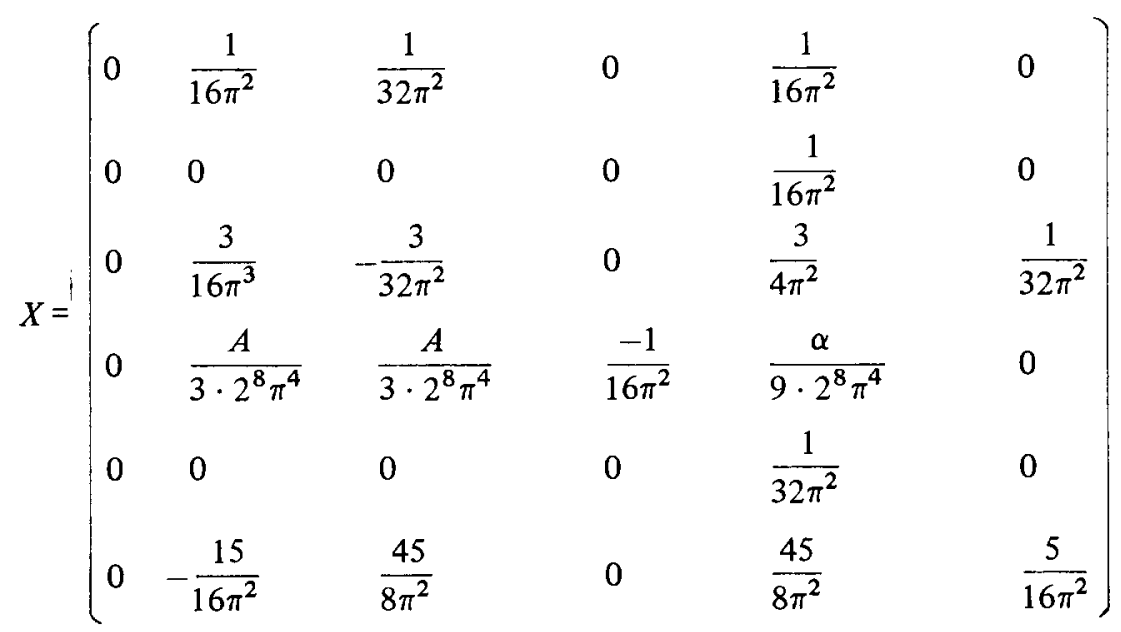

An important check of eq. (164) is provided by evaluating the coefficients $d_{1}^{(1)}$, $d_{1}^{(2)}, d_{1}^{(2)}, d_{1}^{(3)}, d_{1}^{(6)}$ in the series (161), through eq. (164) with the boundary conditions (165). In fact, one obtains

$$
d_{1}^{(1)}=-\frac{i}{3 \cdot 2^{10} \pi^{4}}, \quad d_{1}^{(2)}=-\frac{i}{3 \cdot 2^{10} \pi^{4}}, \quad d_{1}^{(3)}=-\frac{i}{2^{8} \pi^{4}}, \quad d_{1}^{(6)}=-\frac{15 i}{2^{9} \pi^{4}},
$$


which is in complete agreement with the result of the explicit calculations (153). The solution of eq. (164) can be most easily obtained in terms of the effective coupling constant $\bar{\alpha}(\alpha, t)$ defined by

$$
\begin{aligned}
& t=\log \frac{M^{2}}{\mu^{2}} \\
& \frac{\mathrm{d}}{\mathrm{d} t} \bar{\alpha}(t, \alpha)=\frac{3 \bar{\alpha}^{2}}{32 \pi^{2}}, \quad \bar{\alpha}(0, \alpha)=\alpha .
\end{aligned}
$$

The solution of eq. (168) is given by

$$
\bar{\alpha}(t, \alpha)=\frac{\alpha}{1-\frac{3}{32} \alpha t / \pi^{2}} .
$$

Now, the solution of the partial differential equation (164) can be given in the form $(i, j=1, \ldots, 6)$

$$
\begin{aligned}
W_{i} & =\left\{\exp -X \int_{0}^{t} \mathrm{~d} t^{\prime} \bar{\alpha}\left(t^{\prime}, \alpha\right)\right\}_{i j}\left(W_{0}\right)_{j} \\
& =\left\{\exp \left[\frac{32}{3} \pi^{2} X \log \left(1-\frac{3 \alpha}{32 \pi^{2}} \log \frac{M^{2}}{\mu^{2}}\right)\right]\right\}_{i j}\left(W_{0}\right)_{j}
\end{aligned}
$$

with the matrix $X$ given in eq. (166).

Let us now consider case I where $\gamma$ is of the same order with $\alpha$. In this case, one may wish to sum the series

$$
\begin{gathered}
D_{1} \equiv \gamma U_{1}, \quad D_{2} \equiv \gamma U_{2}, \quad D_{3} \equiv \gamma^{2} U_{3}, \quad D_{4} \equiv \gamma^{2} U_{4}, \quad D_{5} \equiv \gamma^{2} U_{5}, \\
D_{6} \equiv \gamma^{3} U_{6}, \quad \bar{D}_{1} \equiv \gamma U_{7}, \quad \bar{D}_{2} \equiv \gamma^{2} U_{8}, \quad \bar{D}_{3} \equiv \gamma^{2} U_{9}, \quad \bar{D}_{1} \equiv \gamma U_{10}, \\
U_{i}=\sum_{k, l=0}^{\infty} d_{k, l}^{(i)} \alpha^{k} \gamma^{l}\left(\log \frac{M^{2}}{\mu^{2}}\right)^{k+l}, \quad(i \neq 6), \\
U_{6}=\sum_{k, l=0}^{\infty} d_{k, l}^{(6)} \alpha^{k} \gamma^{l}\left(\log \frac{M^{2}}{\mu^{2}}\right)^{k+l}+\frac{\alpha}{\gamma} \sum_{k, l=0}^{\infty} e_{k, l}^{(6)} \alpha^{k} \gamma^{l}\left(\log \frac{M^{2}}{\mu^{2}}\right)^{k+l},
\end{gathered}
$$

with the boundary values (see eq. (152))

$$
\begin{array}{ll}
d_{0,0}^{(1)}=d_{0,0}^{(2)}=d_{0,0}^{(3)}=d_{0,0}^{(7)}=e_{0,0}^{(6)}=0, \\
d_{0,0}^{(4)}=\frac{i}{9 \cdot 2^{11} \pi^{4}}, \quad d_{0,0}^{(5)}=\frac{i}{3 \cdot 2^{6} \pi^{2}}, \quad d_{0,0}^{(6)}=-\frac{15 i}{32 \pi^{2}}, \\
d_{0,0}^{(8)}=-\frac{i}{2^{10} \pi^{4}}, \quad d_{0,0}^{(9)}=-\frac{3 i}{32 \pi^{2}}, \quad d_{0,0}^{(10)}=-\frac{i}{64 \pi^{2}} .
\end{array}
$$


Again from the known behavior of the Callan-Symanzik coefficients, it is easy to see that the series (171) correspond to the leading log sum for case I. In terms of the functions $U_{i}(i=1, \ldots, 10)$ introduced in eq. (171), the Callan-Symanzik equations (146)-(148) may be written in the form

$$
\left(M^{2} \frac{\partial}{\partial M^{2}}-\widetilde{B}_{\alpha} \alpha \frac{\partial}{\partial \alpha}-\tilde{t}_{11} \gamma \frac{\partial}{\partial \gamma}\right) U_{i}+P_{i j}(\alpha, \gamma) U_{j}=0,
$$

with the matrix $P(\alpha, \gamma)$ given by formula (174) see page 224 . Inserting the lowest non-trivial-order results for the Callan-Symanzik coefficients and denoting the resulting matrix for $P(\alpha, \gamma)$ by $\vec{P}(\alpha, \gamma)$, the complete leading log sum (171) can be obtained by solving the partial differential equations

$$
\left[M^{2} \frac{\partial}{\partial M^{2}}-\left(\frac{3 \alpha^{2}}{32 \pi^{2}}\right) \frac{\partial}{\partial \alpha}-\left(\frac{\alpha \gamma}{32 \pi^{2}}\right) \frac{\partial}{\partial \gamma}\right] U_{i}+\bar{P}_{i j}(\alpha, \gamma) U_{j}=0
$$

with the boundary conditions

$$
\begin{aligned}
& \left.\left(U_{0}\right)_{i} \equiv U_{i}\right|_{M^{2}=\mu^{2},}, \quad(i=1,2, \ldots, 10), \\
& \left(U_{0}\right)_{1}=\left(U_{0}\right)_{2}=\left(U_{0}\right)_{3}=\left(U_{0}\right)_{7}=0, \\
& \left(U_{0}\right)_{4}=\frac{i}{9 \cdot 2^{11} \pi^{4}}, \quad\left(U_{0}\right)_{5}=\frac{i}{3 \cdot 2^{6} \pi^{2}}, \quad\left(U_{0}\right)_{6}=-\frac{15 i}{32 \pi^{2}}, \\
& \left(U_{0}\right)_{8}=-\frac{i}{2^{10} \pi^{4}}, \quad\left(U_{0}\right)_{9}=-\frac{3 i}{32 \pi^{2}}, \quad\left(U_{0}\right)_{10}=-\frac{i}{64 \pi^{2}} .
\end{aligned}
$$

The explicit expression of the matrix $\vec{P}(\alpha, \gamma)$ is given by formula (177) see page 225 . To solve eq. (175), we may define the effective coupling constants $\bar{\alpha}(t, \alpha)$, $\bar{\gamma}(t, \alpha, \gamma)$ by

$$
\begin{aligned}
& \frac{\mathrm{d}}{\mathrm{d} t} \bar{\alpha}(t, \alpha)=\frac{3 \bar{\alpha}^{2}}{32 \pi^{2}}, \quad \bar{\alpha}(0, \alpha)=\alpha, \\
& \frac{\mathrm{d}}{\mathrm{d} t} \bar{\gamma}(t, \alpha, \gamma)=\frac{\bar{\alpha} \bar{\gamma}}{32 \pi^{2}}, \quad \bar{\gamma}(0, \alpha, \gamma)=\gamma,
\end{aligned}
$$

where $t=\log \left(M^{2} / \mu^{2}\right)$. The solutions to eq. (178) are

$$
\bar{\alpha}(t, \alpha)=\frac{1}{1-\frac{3}{32} \alpha t / \pi^{2}}, \quad \bar{\gamma}(t, \alpha, \gamma)=\gamma\left(1-\frac{3 \alpha}{32 \pi^{2}} \log \frac{M^{2}}{\mu^{2}}\right)^{-1 / 3} .
$$

Now, the solution to eq. (175) can be given in the form 
224

C. Lee / Theory of virtual heavy particle effects

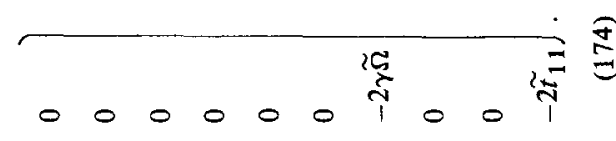

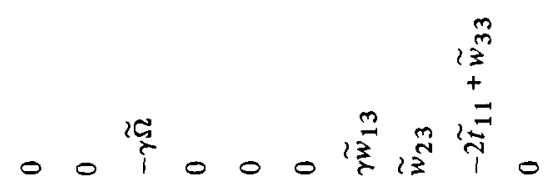
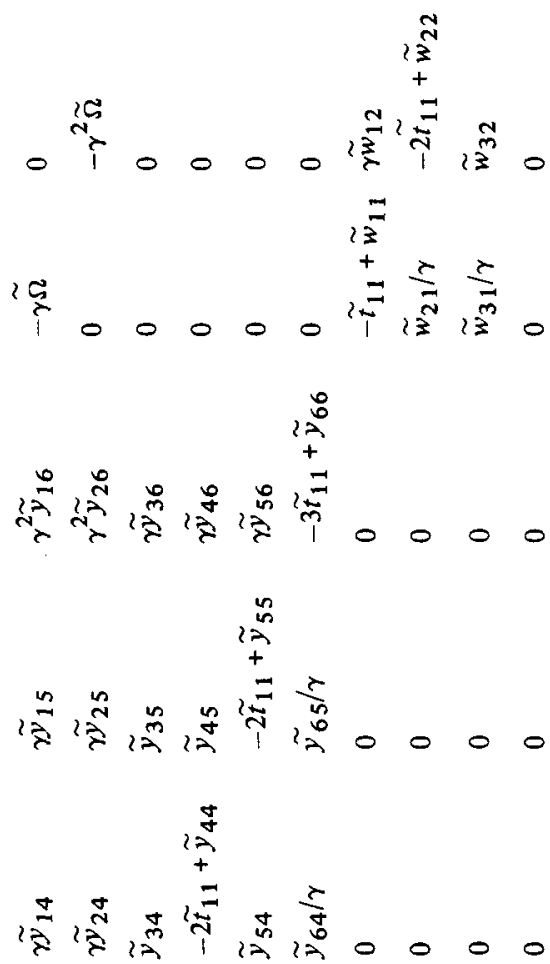

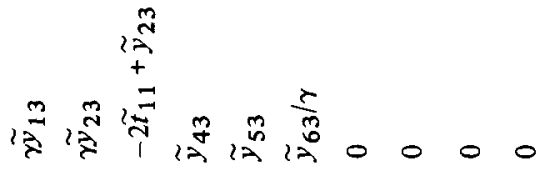

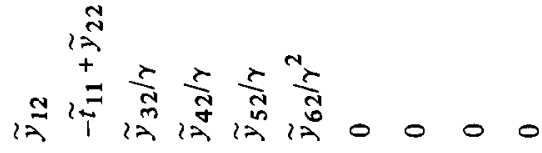

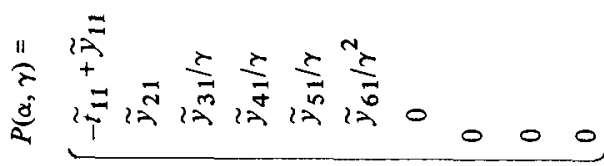


$E$

$$
\begin{aligned}
& 0 \text { 0 } 0 \text { 0 } 0 \text { o }
\end{aligned}
$$

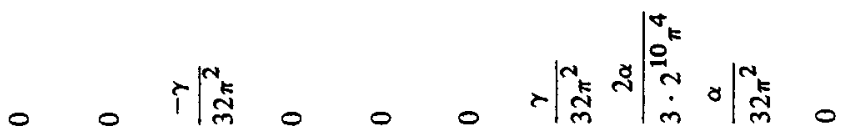

$$
\begin{aligned}
& 00000000000
\end{aligned}
$$

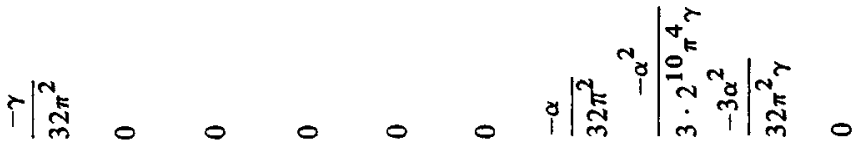

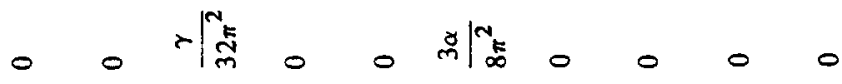

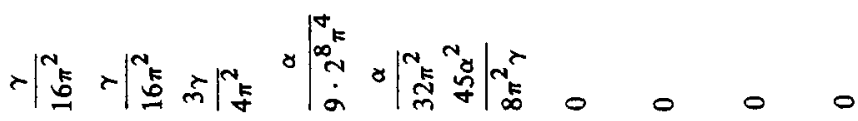

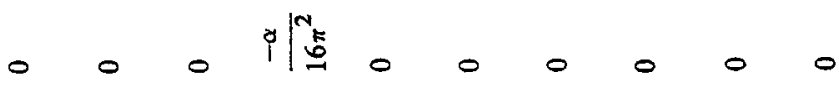

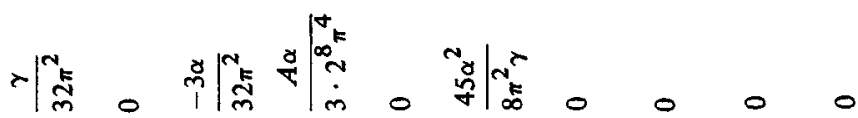

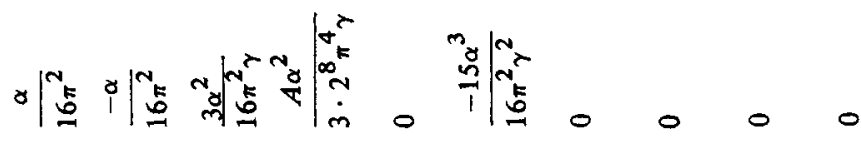

$$
\begin{aligned}
& \left.\checkmark\right|^{N}
\end{aligned}
$$

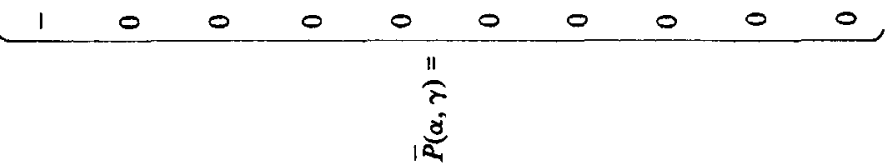




$$
U_{i}=\left\{\exp -\int_{0}^{t} \mathrm{~d} t^{\prime} \mathrm{P}\left(\bar{\alpha}\left(t^{\prime}, \alpha\right), \bar{\gamma}\left(t^{\prime}, \alpha, \gamma\right)\right)\right\}_{i j}\left(U_{0}\right)_{j}, \quad(i, j=1, \ldots, 10)
$$

This completes the discussion of the leading log sum for case I.

Finally, it is amusing to note that, for both cases we have considered, we do not need to know the lowest-order expressions for the effective couplings $D_{1}, D_{2}, D_{3}, \bar{D}_{1}$ as the boundary values. This is quite natural when one notes that $D_{1}, D_{2}, D_{3}, \bar{D}_{1}$ appear with the multiplicative factor $\lambda^{2}$ or $\left(\lambda^{2}\right)^{2}$ in eqs. $(77 \mathrm{a}-\mathrm{c})$. However, these effective couplings must be considered together with $\bar{D}_{1}, \bar{D}_{2}, \bar{D}_{3}, D_{4}, D_{5}, D_{6}$ to obtain a closed set of linear partial differential equations. Of course, once the CallanSymanzik equations (146)-(148) are solved, we may throw away all the auxiliary effective couplings we introduced, namely the functions $D_{1}, D_{2}, D_{3}, \bar{D}_{1}, \bar{D}_{2}, \bar{D}_{3}$ and $\overline{\bar{D}}_{1}$, by setting $\lambda^{2}=\kappa^{2}=0$ in eq. (79).

\section{Discussion}

With a $\phi^{4}$-type field-theory model, we have demonstrated that $O\left(1 / M^{2}\right)$ effects of virtual heavy particles on light-particle Green functions (with all the external momenta much smaller than $M$ ) can be completely factorized via effective local vertices which involve operators of canonical dimension at most six. We have also derived the Callan-Symanzik equations satisfied by the coupling strengths of such effective local interactions, and performed the leading log sum explicitly to illustrate the power of these equations. We believe that the formalism developed in this paper will provide a systematic theoretical language in describing virtual heavy-particle effects in any renormalizable field theory.

It is also a simple matter to generalize the formalism developed in this paper to incorporate the effects of virtual heavy particles accurately up to any given power of $1 / M^{2}$. Suppose we want to identify all the virtual heavy-particle effects up to order $\left(1 / M^{2}\right)^{k}$. For that purpose, one may consider a new oversubtracted function $\bar{H}_{n}\left(p_{1}, \ldots, p_{n}\right)(c f$. , eq. $(20))$ by using an oversubtraction operator $t_{\gamma}^{(2 k)}$ for a proper subgraph corresponding to a $H$-blob. By the power-counting theorem [9], this oversubtracted function will be at most of order $\left(1 / M^{2}\right)^{k+1}$. Then, following the similar line of arguments as given in sect. 2, one can express the difference between the minimally subtracted function and the oversubtracted function via a set of simple effective local vertices. The results may be summarized by

$$
\begin{aligned}
& T_{n, 0}\left(p_{1}, \ldots, p_{n}\right)=\widetilde{T}_{n}\left(p_{1}, \ldots, p_{n}\right)+\widetilde{T}_{n}\left(N\left[\exp ^{\prime} i \int \mathrm{d}^{4} x \mathcal{L}_{\mathrm{eff}}\right], p_{1}, \ldots, p_{n}\right) \\
& \quad+\mathrm{O}\left(\frac{1}{M^{2 k+2}}\right)
\end{aligned}
$$


with the heavy-particle-induced effective lagrangian

$$
\mathscr{L}_{\mathrm{eff}}=\sum_{d=6}^{2 k+4} \sum_{i}\left(\frac{1}{M^{2}}\right)^{d-4} C_{i}^{(d)} \widetilde{O}_{i}^{(d)}(x)
$$

Here, $\widetilde{O}_{i}^{(d)}(x)$ are local, light-particle, operators of canonical dimension $d$ and the calculable constants $C_{i}^{(d)}$ may involve powers of $\log \left(M^{2} / m^{2}\right)$ at most. Also, we used the notation $\exp ^{i} i \int \mathrm{d}^{4} x \mathcal{L}_{\text {eff }}$ in eq. (181) to indicate that the exponential is to be expanded as a Taylor series of $\widetilde{O}_{i}^{(d)}$ 's, and then one should keep only a finite number of lower-order terms in the series, satisfying

$$
\sum_{d} n_{d}(d-4) \leqslant 2 k,
$$

where $n_{d}$ denotes the total number of local operators with canonical dimension $d$ in a given term of the Taylor series. The physical meaning of eq. (181) is clear; to any desired accuracy, the low-energy light-particle physics can be described by a local lagrangian of the form, $\widetilde{\mathcal{L}}+\mathcal{L}_{\text {eff }}$, when combined with appropriate subtraction rules to deal with a single or multiple insertion (s) of $\int \mathrm{d}^{4} x \mathcal{L}_{\text {eff }}$.

Generalization of the present formalism to abelian gauge theory, say QED with heavy muons included, looks straightforward. A new theoretical ingredient here is local gauge invariance, which can be taken into account via the Ward-Takahashi identities. The Ward-Takahashi identities in abelian gauge theories are linear, and thus can be easily incorporated into the derivations of the factorization and Callan-Symanzik equations. As a result, only gauge-invariant effective local vertices will be induced by virtual heavy particles. Also, one may note that there will appear not only dimension-six operators but also dimension-five operators of odd chirality. However, those dimension-five operators must come together with a multiplicative factor $m_{\mathrm{e}}$ (mass of electron), and thus the corresponding coupling strengths will be also of order $1 / M^{2}$ (rather than being of order $1 / M$ ).

Generalization to QCD is less trivial due to complicated nature of non-abelian gauge invariance. The Ward-Takahashi identity is not linear in non-abelian gauge theories, and thus, at least in covariant gauges, effective local vertices involving various null operators may appear [10] Aside from this technical complication, analysis of virtual heavy-quark effects in QCD is very similar to the case of muon in QED. In QCD, it is convenient to use the so-called zero-mass renormalization scheme [11] with respect to light-quark masses and normalize light-particle Green functions at a certain non-zero euclidean momentum $\mu(\mu<<M)$. Then, the generalized subtraction operators $t_{\gamma}^{\prime(0)}, t_{\gamma}^{\prime(2)}$ may be expressed as a Taylor series of lightquark masses and external momenta of a proper subgraph in consideration. This renormalization scheme is especially convenient to deal with the renormalization mixing problem between effective local vertices of opposite chirality. (Here, we emphasize that one should not use the zero-mass renormalization with respect to heavyparticle masses to define light-particle vertex functions. This is necessary for the de- 
coupling theorem to be valid at the zeroth order in $1 / M^{2}$.)

As a sample of imminent physical applications, we may list the effects of heavy quarks (mass $M$ ) in

(i) the total $\mathrm{e}^{+} \mathrm{e}^{-}$annihilations; $\mathrm{e}^{+} \mathrm{e}^{-} \rightarrow \gamma(q) \rightarrow$ (hadrons), and

(ii) the inclusive electron scattering off a light hadron;

$$
\gamma(q)+|p\rangle \rightarrow \text { (hadrons), for fixed } \frac{-q^{2}}{2 p \cdot q}=x .
$$

(See figs. 12a, b, and also fig. 13a, b for charged heavy quarks) Conventionally, one analyzes these processes first by looking at the time-ordered products of electromagnetic currents, i.e.,

$$
\begin{array}{ll}
\int \mathrm{d}^{4} x \mathrm{e}^{-i q \cdot x}\left\langle 0\left|\mathrm{~T}\left(J_{\mu}(x) J_{\nu}(0)\right)\right| 0\right\rangle, & \text { for process (i) } \\
\int \mathrm{d}^{4} x \mathrm{e}^{-i q \cdot x}\left\langle\mathrm{p}\left|\mathrm{T}\left(J_{\mu}(x) J_{\nu}(0)\right)\right| \mathrm{p}\right\rangle, & \text { for process (ii) }
\end{array}
$$

and then uses the unitarity relation. Of course, the method of analysis crucially depends on the relative magnitudes of $q^{2}, M^{2}$, and all the other hadronic scales which we may simply represent by $\mu^{2}$. The cases of $q^{2}>M^{2}>\mu^{2}$ and $q^{2} \sim M^{2} \gg \mu^{2}$ have been discussed in a recent paper by Witten [12]. Here, we concentrate on the case of $M^{2} \gg q^{2} \gg \mu^{2}$, where the formalism developed in the present paper should be used for the analysis. As mentioned earlier, the zero-mass renormalizations may be used with respect to light-quark masses only. Now, using the formalism developed in the present paper, one may first isolate all the $\mathrm{O}\left(1 / M^{2}\right)$ effects in terms of effective local vertices (which may include photon fields for charged heavy quarks), and then sum the powers of $\log M^{2}$ by the method given in sect. 3 (the first factorization). For $q^{2} \gg \mu^{2}$, effective vertices involving dimension-six (rather than dimension-five) operators will dominate, yielding contributions

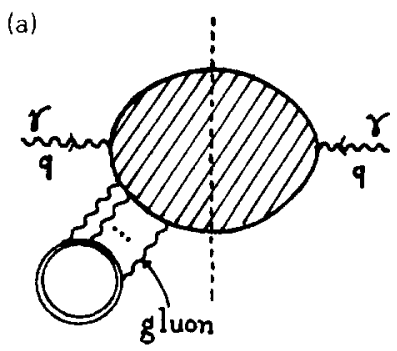

(b)

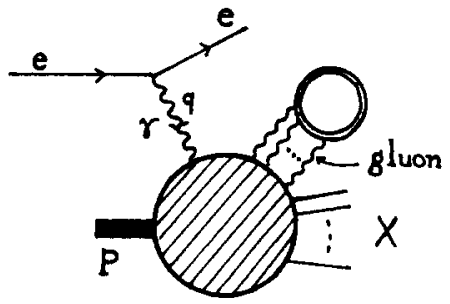

Fig. 12(a) Heavy quarks in total $\mathrm{e}^{+} \mathrm{e}^{-}$annihilation. The double solid line indicates the heavyquark line. (b) Heavy quarks in deep inelastic electron scattering. 
(a)

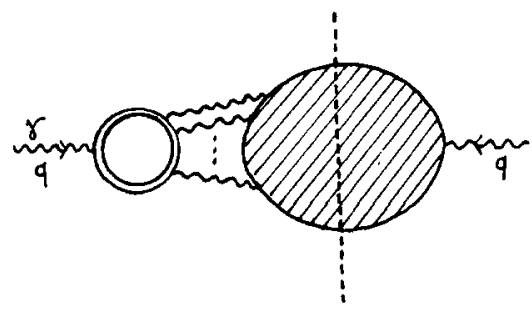

(b)

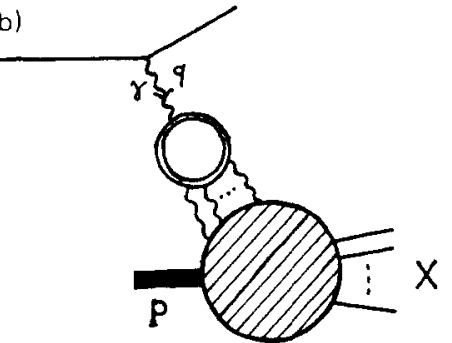

Fig. 13(a) Charged heavy quarks in total $\mathrm{e}^{+} \mathrm{e}^{-}$annihilation. (b) Charged heavy quarks in deep inelastic electron scattering.

of order $q^{2} / M^{2}$, compared to the contributions obtained with heavy quarks completely ignored. To sum the powers of $\log q^{2}$ in the case of the function (184a), one may directly apply the renormalization group corresponding to the variation $\mu^{2} \widetilde{D} / D \mu^{2}$, for the vertex functions with such effective local vertices. As for the function (184b), one should first write down the light-cone expansion for the vertex functions with such effective local vertices, in terms of twist-two local operators. (The second factorization). Then, one may use the renormalization group corresponding to the variation $\mu^{2} \widetilde{D} / D \mu^{2}$, to sum the powers of $\log q^{2}$. Detailed analyses may be given elsewhere.

Yet, the most interesting applications of our formalism presumably lie in the study of unified or grand unified gauge theories. To investigate such theories, we have to generalize the present formalism to spontaneously broken theories in which even the virtual heavy particles in consideration acquire mass via spontaneous symmetry breaking. We have studied the two simple field theories: the linear $\sigma$-model in the Goldstone mode and the abelian Higgs model with light fermions, and found that simple local structures do exist for both cases [14]. For the former in the limit of large mass for $S=\sigma-\langle\sigma\rangle$ and with fixed quartic coupling (this implies also a large number for $\langle\sigma\rangle)$, the low-energy pion theory can be described by the effective lagrangian of the form (182), with the restriction (183). But, there appear some special features like: (i) the zeroth-order lagrangian, i.e., $\widetilde{\mathcal{L}}$, is given by the free massless pion theory; (ii) among the operators $\widetilde{O}_{i}^{(d)}$ with an equal number of pion fields, the effective vertices involving the least number (here, it is two) of derivatives are uniquely determined by the non-linear $\sigma$-model [15]. In the abelian Higgs model, the low-energy effective fermion theory does not show any sign of underlying local gauge symmetry. These results seem to suggest that our formalism will also be useful in unified gauge theories, but, clearly, more investigations are necessary.

We would like to thank Prof. Y.P. Yao who first told us about the possibility of factorization in studying virtual heavy-particle effects. Without his comment, this 
research would not have started, and we are grateful to him for suggesting this problem and helpful discussions.

\section{Appendix A}

Here we derive the factorizations (109) and (110), respectively for the vertex functions $\Gamma_{2,2}^{\prime}\left(p_{1}, p_{2}, P_{1}, P_{2} ; \lambda^{2}, \kappa^{2}\right)$ and $\left(\partial^{2} / \partial\left(\lambda^{2}\right)^{2}\right) \Gamma_{0,2}^{\prime}\left(P,-P ; \lambda^{2}, \kappa^{2}\right)$. In the $(\phi-\psi)^{\prime}$ theory, these vertex functions are given by the forest formulae [7]

$$
\begin{aligned}
& \Gamma_{2,2}^{\prime}\left(p_{1}, p_{2}, P_{1}, P_{2} ; \lambda^{2}, \kappa^{2}\right)=\sum_{\Omega} \prod_{\gamma \in \Omega}\left(-t_{\gamma}^{\prime(0)}\right) \Gamma_{2,2}^{\prime \mathrm{u}}\left(p_{1}, p_{2}, P_{1}, P_{2} ; \lambda^{2}, \kappa^{2}\right) \\
& \frac{\partial^{2}}{\partial\left(\lambda^{2}\right)^{2}} \Gamma_{0,2}^{\prime}\left(P,-P ; \lambda^{2}, \kappa^{2}\right)=\frac{\partial^{2}}{\partial\left(\lambda^{2}\right)^{2}}\left[\sum_{\Omega}^{\text {normal }} \prod_{\gamma \in \Omega}\left(-t_{\gamma}^{\prime(0)}\right) \Gamma_{0,2}^{\prime \mathrm{u}}\left(P,-P ; \lambda^{2}, \kappa^{2}\right)\right]
\end{aligned}
$$

To derive the desired factorizations, it is convenient to introduce a $\Omega^{\prime}$ forest which is defined by adding the $(2+2)$-point tree vertex $\gamma_{0}$ (see figs. $14 \mathrm{a}, \mathrm{b}$ for examples) as a possible element of a forest besides usual non-overlapping proper subgraphs. (Note that, according to our definition, a point vertex is not considered as a proper subgraph.) Then the forest formulae (A.1) and (A.2) may be written as

$$
\begin{aligned}
& \Gamma_{2,2}^{\prime}\left(p_{1}, p_{2}, P_{1}, P_{2} ; \lambda^{2}, \kappa^{2}\right)=\sum_{\Omega^{\prime}} \sum_{\gamma \in \Omega^{\prime}}\left(-t_{\gamma}^{\prime(0)}\right) \Gamma_{2,2}^{\prime \mathrm{u}}\left(p_{1}, p_{2}, P_{1}, P_{2} ; \lambda^{2}, \kappa^{2}\right), \\
& \frac{\partial^{2}}{\partial\left(\lambda^{2}\right)^{2}} \Gamma_{0,2}^{\prime}\left(P,-P ; \lambda^{2}, \kappa^{2}\right)=\frac{\partial^{2}}{\partial\left(\lambda^{2}\right)^{2}}\left[\sum_{\Omega^{\prime}}^{\text {normal }} \prod_{\gamma \in \Omega^{\prime}}\left(-t_{\gamma}^{\prime(0)}\right) \Gamma_{0,2}^{\prime \mathrm{u}}\left(P,-P ; \lambda^{2}, \kappa^{2}\right)\right],
\end{aligned}
$$

assuming $t_{\gamma}^{\prime(0)}=0$ for $\gamma=\gamma_{0}$.

We now introduce the oversubtracted functions

$$
\bar{\Gamma}_{2,2}^{\prime}\left(p_{1}, p_{2}, P_{1}, P_{2} ; \lambda^{2}, \kappa^{2}\right)=\sum_{\Omega^{\prime}} \sum_{\gamma \in \Omega^{\prime}}\left(-\bar{t}_{\gamma}^{\prime}\right) \Gamma_{2,2}^{\prime u}\left(p_{1}, p_{2}, P_{1}, P_{2} ; \lambda^{2}, \kappa^{2}\right),
$$
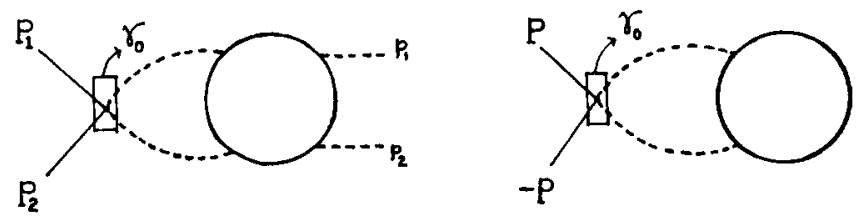

Fig. 14. Illustrations of the tree vertex $\gamma_{0}$. 


$$
\overline{\left(\frac{\partial^{2}}{\partial\left(\lambda^{2}\right)^{2}} \Gamma_{0,2}^{\prime}\left(P,-P ; \lambda^{2}, \kappa^{2}\right)\right)}=\frac{\partial^{2}}{\partial\left(\lambda^{2}\right)^{2}}\left[\sum_{\Omega^{\prime}} \sum_{\gamma \in \Omega^{\prime}}\left(-\bar{t}_{\gamma}^{\prime}\right) \Gamma_{0,2}^{\prime u}\left(P,-P ; \lambda^{2}, \kappa^{2}\right)\right],
$$

where the Taylor operator $\bar{t}_{\gamma}^{\prime}$ is specified by

$$
\begin{array}{ll}
\bar{t}^{\prime} \Gamma_{2,2}^{\prime \mathrm{u}}=-i \gamma, & \text { if the element } \gamma \text { corresponds } \\
& \text { to } \gamma_{0} \text { and there are no internal } \psi \text {-lines left } \\
& \text { in any part of the graph, } \\
\bar{t}_{\gamma}^{\prime}=t_{\gamma}^{\prime(0)}, & \text { otherwise } .
\end{array}
$$

Now, going through the same argument as given in sect. 2 , it is a simple matter to obtain the following forms of the algebraic identity

$$
\begin{aligned}
& \Gamma_{2,2}^{\prime}\left(p_{1}, p_{2} ; \lambda^{2}, \kappa^{2}\right)=-i \gamma \tilde{\Gamma}_{2}^{\prime}\left(\frac{1}{2} N\left[\phi^{2}(x=0)\right], p_{1}, p_{2} ; \lambda^{2}\right) \\
& \quad+\bar{\Gamma}_{2,2}^{\prime}\left(p_{1}, p_{2}, P_{1}, P_{2} ; \lambda^{2}, \kappa^{2}\right), \\
& \frac{\partial^{2}}{\partial\left(\lambda^{2}\right)^{2}} \Gamma_{0,2}^{\prime}\left(P,-P ; \lambda^{2}, \kappa^{2}\right)=-i \gamma \frac{\partial^{2}}{\partial\left(\lambda^{2}\right)^{2}} \tilde{\Gamma}_{0}^{\prime}\left(\frac{1}{2} N\left[\phi^{2}(x=0)\right], \lambda^{2}\right) \\
& \quad+\sqrt{\left(\frac{\partial^{2}}{\partial\left(\lambda^{2}\right)^{2}} \Gamma_{0,2}^{\prime}\left(P,-P ; \lambda^{2}, \kappa^{2}\right)\right)},
\end{aligned}
$$

where we introduced the local operator $\frac{1}{2} N\left[\phi^{2}(x=0)\right]$ (see fig. 15 for the tree graph) in the $\phi^{\prime}$ theory and $\widetilde{\Gamma}_{0}^{\prime}$ denotes its vacuum matrix element. In eqs. (A.8) and (A.9), we have defined

$$
\begin{aligned}
& \widetilde{\Gamma}_{2}^{\prime}\left(\frac{1}{2} N\left[\phi^{2}(x=0)\right], p_{1}, p_{2} ; \lambda^{2}\right)=\sum_{\Omega} \prod_{\gamma \in \Omega}\left(-t_{\gamma}^{\prime(0)}\right) \tilde{\Gamma}_{2}^{\prime u}\left(\frac{1}{2} \phi^{2}(x=0), p_{1}, p_{2} ; \lambda^{2}\right), \\
& \frac{\partial^{2}}{\partial\left(\lambda^{2}\right)^{2}} \widetilde{\Gamma}_{0}^{\prime}\left(\frac{1}{2} N\left[\phi^{2}(x=0)\right] ; \lambda^{2}\right)=\frac{\partial^{2}}{\partial\left(\lambda^{2}\right)^{2}} \sum_{\Omega}^{\text {normal }} \prod_{\gamma \in \Omega}\left(-t_{\gamma}^{\prime(0)}\right) \widetilde{\Gamma}_{0}^{\prime u}\left(\frac{1}{2} \phi^{2}(x=0) ; \lambda^{2}\right)
\end{aligned}
$$

with the Taylor operator $t_{\gamma}^{\prime(0)}$ specified by eqs. $(40 \mathrm{a}-\mathrm{c})$ and

$$
\begin{aligned}
& t^{\prime(0)} \widetilde{\Gamma}_{2}^{\mathrm{u}}\left(\frac{1}{2} \phi^{2}(x=0), q_{1}, q_{2} ; \lambda^{2}\right)=\widetilde{\Gamma}_{2}^{\prime \mathrm{u}}\left(\frac{1}{2} \phi^{2}(x=0), q_{1}, q_{2} ; \lambda^{2}\right) \underset{q i=0, \lambda^{2}=0}{\mid}, \\
& t^{\prime(0)} \widetilde{\Gamma}_{k}^{\prime \mathrm{u}}\left(\frac{1}{2} \phi^{2}(x=0), q_{1}, \ldots, q_{k} ; \lambda^{2}\right)=0, \quad(k>2) .
\end{aligned}
$$

The forest formula (A.10) implies the normalization

$$
\tilde{\Gamma}_{2}^{\prime}\left(\frac{1}{2} N\left[\phi^{2}(x=0)\right], p_{1}, p_{2} ; \lambda^{2}\right) \underset{p_{i}=0, \lambda^{2}=0}{\mid}=1 .
$$




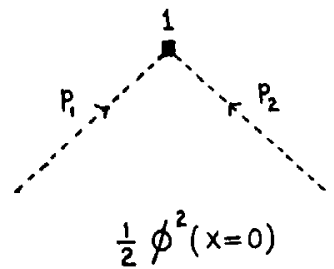

Fig. 15. Tree graph for $\frac{1}{2} \phi^{2}(x=0)$.

If $P_{1}, P_{2}, P$ are large space-like momenta, it is a matter of simple power counting [9] to show that when $-P_{1}^{2},-P_{2}^{2},-P^{2}, M^{2}$ are much larger than the masses $\mu^{2}, \lambda^{2}, \kappa^{2}$ and the momenta of the external $\phi$-legs, the oversubtracted functions in the righthand sides of eqs. (A.8) and (A.9) are at most of $O\left(1 / M^{2}\right)$. This proves the factorizations (109) and (110), for the space-like momenta $P_{1}, P_{2}, P$. For large time-like momenta close to the heavy-particle mass-shell, one may worry about the integration region where the momentum of a certain internal $\psi$-line stays close to its mass-shell. (Note that, by construction, Feynman graphs for the above oversubtracted functions always possess a non-vanishing number of internal $\psi$-lines.) However, in the scalar field theory being considered in this paper, it is easy to convince oneself that such 'infrared' contributions to the above oversubtracted functions are at most of order $1 / M$. Thus, the factorizations (109) and (110) are also valid for the large time-like momenta.

\section{Appendix B}

In the main text, we have normalized the primitively divergent vertex functions involving light particles at zero external momenta for those light particles, for the sake of simplicity. Here we briefly indicate necessary modifications when different normalization conditions are employed. Suppose we have normalized the $\phi-\psi$ theory by imposing

$$
\begin{aligned}
& \left.\Gamma_{2,0}(p,-p)\right|_{p} ^{2}=\mu^{2}=0, \\
& \left.\frac{\partial}{\partial p^{2}} \Gamma_{2,0}(p,-p)\right|_{p^{2}-\mu^{2}}=i \rho_{\phi}, \\
& \Gamma_{4,0}\left(p_{1}, p_{2}, p_{3}, p_{4}\right) \\
& \left.p_{i}^{2}\right|_{\mu} ^{2} \quad=-i \alpha \rho_{\phi}^{2}, \\
& \left(p_{1}+p_{2}\right)^{2}=\left(p_{2}+p_{3}\right)^{2}=\left(p_{1}+p_{3}\right)^{2}=\frac{4}{3} \mu^{2} \\
& \Gamma_{2,2}\left(p_{1}, p_{2}, P_{1}, P_{2}\right) \quad p_{1}^{2}=p_{2}^{2}=\mu^{2}, P_{1}^{2}=P_{2}^{2}=M^{2}=-i \gamma \rho_{\psi} \rho_{\phi} \text {, } \\
& \left(p_{1} \dagger p_{2}\right)^{2}=4 \mu^{2},\left(p_{1}+P_{1}\right)^{2}=\left(p_{1}+P_{2}\right)^{2}=M^{2}-\mu^{2}
\end{aligned}
$$


instead of the conditions ( $4 \mathrm{a}, \mathrm{b}, \mathrm{e}, \mathrm{g}$ ), and the $\phi$-theory by imposing

$$
\begin{aligned}
& \left.\widetilde{\Gamma}_{2}(p,-p)\right|_{p^{2}=\mu^{2}}=0, \\
& \frac{\partial}{\partial p^{2}} \widetilde{\Gamma}_{2}(p,-p)_{p^{2}=\mu^{2}}^{\mid}=i \rho_{\phi}, \\
& \widetilde{\Gamma}_{4}\left(p_{1}, p_{2}, p_{3}, p_{4}\right) \underset{\left(p_{1}+p_{2}\right)^{2}=\left(p_{2}+p_{3}\right)^{2}=\left(p_{1}+p_{3}\right)^{2}=\frac{4}{3} \mu^{2}}{p_{i}^{2}=\mu^{2}}
\end{aligned}
$$

instead of the conditions $(7 \mathbf{a}-\mathrm{c})$. In terms of Zimmermann's subtraction language, these on-shell normalization conditions can be achieved by using the Taylor expansion operator around the mass-shell. For instance, in the $\phi$-theory the Taylor operator $t_{\gamma}^{(0)}$ in the Forest formula (14) is now given by

$$
\begin{aligned}
& t^{(0)} \widetilde{\Gamma}_{2}^{\mathrm{u}}(q,-q)=\widetilde{\Gamma}_{2}^{\mathrm{u}}(q,-q) \underset{q^{2}=\mu^{2}}{1}+\left(q^{2}-\mu^{2}\right)\left[\frac{\partial}{\partial q^{2}} \widetilde{\Gamma}_{2}^{\mathrm{u}}(q,-q)\right]_{q^{2}=\mu^{2}}, \\
& t^{(0)} \tilde{\Gamma}_{4}^{\mathrm{u}}\left(q_{1}, q_{2}, q_{3}, q_{4}\right)=\widetilde{\Gamma}_{4}^{\mathrm{u}}\left(q_{1}, \ldots, q_{4}\right) \quad \\
& t^{(0)} \tilde{\Gamma}_{k}^{\mathrm{u}}\left(q_{1}, \ldots, q_{k}\right)=0, \quad \begin{array}{c}
q_{i}^{2}=\mu^{2} \\
\left(q_{1}+q_{3}\right)^{2}=\left(q_{2}+q_{3}\right)^{2}=\left(q_{1}+q_{3}\right)^{2}=\frac{4}{3} \mu^{2}
\end{array},
\end{aligned}
$$

To prove the factorization, we may again introduce the oversubtracted function $\bar{H}_{n}\left(p_{1}, \ldots, p_{n}\right)$ by the forest formula (20). With the imposition of the normalization conditions (B.1) and (B.2), we may specify the oversubtraction operator $\bar{t}_{\gamma}$ (see eq. (21)) by using the Taylor operator $t_{\gamma}^{(2)}$ defined by

$$
\begin{aligned}
& t^{(2)} H_{2}^{\mathrm{u}}(q,-q)=H_{2}^{\mathrm{u}}(q,-q) \underset{q^{2}=\mu^{2}}{l^{2}}+\left(q^{2}-\mu^{2}\right)\left[\frac{\partial}{\partial q^{2}} H_{2}^{\mathrm{u}}(q,-q)\right]_{q^{2}=\mu^{2}} \\
& +\frac{1}{2}\left(q^{2}-\mu^{2}\right)^{2}\left[\frac{\partial^{2}}{\partial\left(q^{2}\right)^{2}} H_{2}^{\mathrm{u}}(q,-q)\right]_{q^{2}=\mu^{2}}, \\
& t^{(2)} H_{4}^{\mathrm{u}}\left(q_{1}, q_{2}, q_{3}, q_{4}\right)=H_{4}^{\mathrm{u}}\left(q_{i}^{2}, s, t\right) \underset{\substack{q_{i}^{2}=\mu^{2} \\
s=t=\frac{4}{3} \mu^{2}}}{\mid} \\
& +\sum_{i=1}^{4}\left(q_{i}^{2}-\mu^{2}\right)\left[\frac{\partial}{\partial q_{i}^{2}} H_{4}^{\mathrm{u}}\left(q_{i}^{2}, s, t\right)\right] \underset{\substack{q_{i}^{2}=\mu^{2} \\
s=t=\frac{4}{3} \mu^{2}}}{1}
\end{aligned}
$$




$$
\begin{aligned}
& +\left(s-\frac{4}{3} \mu^{2}\right)\left[\frac{\partial}{\partial S} H_{4}^{\mathrm{u}}\left(q_{i}^{2}, s, t\right)\right]_{\substack{q_{i}^{2}=\mu^{2} \\
s=t=\frac{4}{3} \mu^{2}}}+\left(t-\frac{4}{3} \mu^{2}\right) \\
& \times\left[\frac{\partial}{\partial t} H_{4}^{\mathrm{u}}\left(q_{i}^{2}, s, t\right)\right]_{\substack{q_{i}^{2}=\mu^{2} \\
s=t=\frac{4}{3} \mu^{2}}}, \\
& t^{(2)} H_{6}^{\mathrm{u}}\left(q_{1}, \ldots, q_{6}\right)=\left.H_{6}^{\mathrm{u}}\left(q_{1}, \ldots q_{6}\right)\right|_{q_{i}=0}, \\
& t^{(2)} H_{k}^{\mathrm{u}}\left(q_{1}, \ldots, q_{k}\right)=0, \quad(k>6) .
\end{aligned}
$$

(Here, we have assumed that the four-point functions $H_{4}^{\mathrm{u}}$ are written in terms of Lorentz scalars, i.e.,

$$
H_{4}^{\mathrm{u}}\left(q_{1}, q_{2}, q_{3}, q_{4}\right) \equiv H_{4}^{\mathrm{u}}\left(q_{1}^{2}, q_{1}^{2}, q_{3}^{2}, q_{4}^{2}, s, t\right)
$$

with

$$
\left.s=\left(q_{1}+q_{2}\right)^{2}, \quad t=\left(q_{2}+q_{3}\right)^{2}, \quad u=\left(q_{1}+q_{3}\right)^{2}=\sum_{i=1}^{4} q_{i}^{2}-s-t .\right)
$$

With these definitions, the proof of the factorization in sect. 2 goes through entirely with the following effective local vertices

$$
\begin{aligned}
& \widetilde{O}_{1}=\frac{1}{2} \rho_{\phi} \int \mathrm{d}^{4} x N\left[\phi\left(\partial^{2}+\mu^{2}\right)^{2} \phi(x)\right], \\
& \widetilde{O}_{2}=-\frac{1}{6} \rho_{\phi}^{2} \int \mathrm{d}^{4} x N\left[\phi^{3}\left(\partial^{2}+\mu^{2}\right) \phi(x)\right], \\
& \widetilde{O}_{3}=\frac{\rho_{\phi}^{3}}{6 !} \int \mathrm{d}^{4} x N\left[\phi^{6}(x)\right],
\end{aligned}
$$

Tree vertices for $\widetilde{O}_{1}, \widetilde{\mathcal{O}}_{2}, \widetilde{\mathrm{O}}_{3}$ are shown in fig. 16 . Vertex functions with these effec-

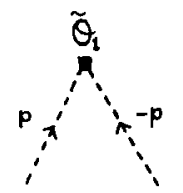

$$
p_{\phi}\left(p^{2}-\mu^{2}\right)^{2}
$$

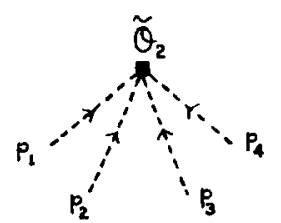

$$
\rho_{\phi}^{2}\left(p_{1}^{2}+p_{2}^{2}+p_{3}^{2}+p_{4}^{2}-4 \mu^{2}\right)
$$

Fig. 16. Tree vertices for $\widetilde{O}_{1}, \widetilde{O}_{2}, \widetilde{O}_{3}$.

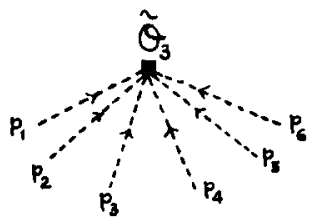

$\rho_{\phi}^{3}$ 
tive vertices once inserted should be minimally renormalized and satisfy the normalization conditions analogous to eqs. $(11 \mathrm{a}-\ell)$, this time specified on the mass-shell.

To derive the Callan-Symanzik equations, we may introduce $\lambda^{2}$ and $\kappa^{2}$ as discussed in sect. 3 and choose all the renormalization counterterms such that they are independent of $\lambda^{2}$ and $\kappa^{2}$. Here, the normalization conditions (41a-d) for the $\phi^{\prime}$ theory should be replaced by

$$
\begin{aligned}
& \widetilde{\Gamma}_{2}^{\prime}\left(p,-p ; \lambda^{2}\right) \underset{p^{2}=\mu^{2}, \lambda^{2}=0}{\mid}=0, \\
& \frac{\partial}{\partial p^{2}} \widetilde{\Gamma}_{2}^{\prime}\left(p,-p ; \lambda^{2}\right) \underset{p^{2}=\mu^{2}, \lambda^{2}=0}{\mid}=i \rho_{\phi}, \\
& \frac{\partial}{\partial \lambda^{2}} \widetilde{\Gamma}_{2}^{\prime}\left(p,-p, \lambda^{2}\right) \underset{p^{2}=\mu^{2}, \lambda^{2}=0}{\mid}=-i \rho_{\phi}, \\
& \left.\widetilde{\Gamma}_{4}^{\prime}\left(p_{1}, p_{2}, p_{3}, p_{4} ; \lambda^{2}\right)\right|_{\substack{\lambda^{2}=0, p_{i}^{2}=\mu^{2} \\
S=t=u=\frac{4}{3} \mu^{2}}} ^{\mid}=-i \alpha \rho_{\phi}^{2},
\end{aligned}
$$

and the normalization conditions $(57 \mathrm{a}-\mathrm{k})$ by similar replacements evaluated on the mass-shell. Then, the Callan-Symanzik equation of the form (51) or (65) follows in a straightforward manner if one varies $\mu^{2}$ for fixed $\tilde{m}_{\mathrm{B}}^{2}, \tilde{\rho}_{\mathrm{B} \phi}, \widetilde{\alpha}_{\mathrm{B}}, \Lambda^{2}$ (in the $\phi^{\prime}$ theory) or for fixed $m_{\mathrm{B}}^{2}, M^{2}, M_{\mathrm{B}}^{2}, \rho_{\mathrm{B} \phi}, \rho_{\mathrm{B} \psi}, \alpha_{\mathrm{B}}, \beta_{\mathrm{B}}, \gamma_{\mathrm{B}}, \Lambda^{2}$ (in the $(\phi-\psi)^{\prime}$ theory).

For the limiting case of $\mu, \lambda, \kappa, p_{1}, \ldots, p_{n}<<M$ in the $(\phi-\psi)^{\prime}$ theory, factorization of the form (79) will be still valid if we replace the effective vertices $\widetilde{O}_{i}^{\prime}(i=$ $1, \ldots 6)$ with

$$
\begin{aligned}
& \widetilde{O}_{1}=\frac{1}{2} \rho_{\phi} \int \mathrm{d}^{4} x N\left[\phi^{2}(x)\right] \\
& \widetilde{\mathcal{O}}_{2}^{\prime}=-\frac{1}{2} \rho_{\phi} \int \mathrm{d}^{4} x N\left[\phi\left(\partial^{2}+\mu^{2}\right) \phi(x)\right], \\
& \widetilde{\mathcal{O}}_{3}^{\prime}=\frac{\rho_{\phi}^{2}}{4 !} \int \mathrm{d}^{4} x N\left[\phi^{4}(x)\right] \\
& \widetilde{O}_{4}^{\prime}=\frac{1}{2} \rho_{\phi} \int \mathrm{d}^{4} x N\left[\phi\left(\partial^{2}+\mu^{2}\right)^{2} \phi(x)\right] \\
& \widetilde{O}_{5}^{\prime}=-\frac{1}{6} \rho_{\phi}^{2} \int \mathrm{d}^{4} x N\left[\phi^{3}\left(\partial^{2}+\mu^{2}\right) \phi(x)\right] \\
& \widetilde{O}_{6}^{\prime}=\frac{\rho_{\phi}^{3}}{6 !} \int \mathrm{d}^{4} x N\left[\phi^{6}(x)\right] .
\end{aligned}
$$


Again, vertex functions with these vertices once inserted are assumed to be minimally subtracted (i.e., use the operator $t_{\gamma}^{\prime(0)}$, defined by the Taylor series expansion in $\lambda^{2}, k^{2}$ and momentum variables around the mass-shell).

Now, we comment on the derivation of the Callan-Symanzik equations satisfied by $\widetilde{\Gamma}_{n}^{\prime}\left(\widetilde{O}_{i}^{\prime}, p_{1}, \ldots, p_{n} ; \lambda^{2}\right)$. For $\widetilde{O}_{1}^{\prime}$, the situation is identical to that in the main text, with $\widetilde{\Gamma}_{n}^{\prime u}\left(\widetilde{O}_{1}^{\prime}, p_{1}, \ldots, p_{n} ; \tilde{m}_{\mathrm{B}}^{2}, \tilde{\rho}_{\mathrm{B} \phi}, \widetilde{\alpha}_{\mathrm{B}}, \Lambda^{2}\right)$ being invariant under the variation $\mu^{2} \widetilde{D} / D \mu^{2}$. However, starting from $\widetilde{O}_{2}^{\prime}$, the situation is a bit different due to explicit $\mu^{2}$ dependence of the $\widetilde{O}_{2}^{\prime}$ vertex. This can be resolved as follows. Let us denote the function obtained from the unsubtracted function $\widetilde{\Gamma}_{n}^{\prime u}\left(\widetilde{O}_{2}^{\prime u}, p_{1}, \ldots, p_{n} ; \lambda^{2}\right)$ by replacing $\mu^{2}+\lambda^{2}$ (appearing in propagators), $\rho_{\phi}, \alpha$ with $\widetilde{m}_{\mathrm{B}}^{2}, \widetilde{\rho}_{\mathrm{B} \phi}, \widetilde{\alpha}_{\mathrm{B}}$, as $\widetilde{\Gamma}_{n}^{\prime \mathrm{u}}\left(\widetilde{\mathrm{O}}_{2}^{\prime}, p_{1}, \ldots, p_{n}\right.$; $\left.\mu^{2}, \widetilde{m}_{\mathrm{B}}^{2}, \tilde{\rho}_{\mathrm{B} \phi}, \widetilde{\alpha}_{\mathrm{B}}, \Lambda^{2}\right)$. (Here, the explicit $\mu^{2}$ dependence in $\widetilde{\Gamma}_{n}^{\prime \mathrm{u}}\left(\widetilde{\sigma}_{2}^{\prime}, p_{1}, \ldots, p_{n} ; \mu^{2}\right.$, $\tilde{m}_{\mathrm{B}}^{2}, \widetilde{\rho}_{\mathrm{B} \phi}, \widetilde{\alpha}_{\mathrm{B}}, \Lambda^{2}$ ) comes from the $\widetilde{\mathcal{O}}_{2}^{\prime}$ vertex.) Then,

$$
\begin{aligned}
& \tilde{\Gamma}_{n}^{\prime \mathrm{u}}\left(\tilde{O}_{2}^{\prime}, p_{1}, \ldots, p_{n} ; \tilde{m}_{\mathrm{B}}^{2}, \tilde{m}_{\mathrm{B}}^{2}, \tilde{\rho}_{\mathrm{B} \phi}, \tilde{\alpha}_{\mathrm{B}}, \Lambda^{2}\right) \\
& =\widetilde{\Gamma}_{n}^{\prime \mathbf{u}}\left(\widetilde{\widetilde{O}}_{2}^{\prime}, p_{1}, \ldots, p_{n} ; \mu^{2}, \tilde{m}_{\mathbf{B}}^{2}, \tilde{\rho}_{\mathbf{B} \phi}, \widetilde{\alpha}_{\mathbf{B}}, \Lambda^{2}\right) \\
& +\left(\tilde{\mu}_{\mathrm{B}}^{2}-\mu^{2}+\lambda^{2} \widetilde{Z}_{\lambda}\right) \widetilde{\Gamma}_{n}^{\prime \mathrm{u}}\left(\widetilde{O}_{1}^{\prime}, p_{1}, \ldots, p_{n} ; \tilde{m}_{\mathrm{B}}^{2}, \widetilde{\rho}_{\mathrm{B} \phi}, \widetilde{\alpha}_{\mathrm{B}}, \Lambda^{2}\right)
\end{aligned}
$$

is invariant under the variation $\mu^{2} \widetilde{D} / D \mu^{2}$. Now, the desired equation can be obtained if one re-expresses the right-hand side of eq. (B.9) in terms of renormalized vertex functions and then takes the derivative $\mu^{2} \widetilde{D} / D \mu^{2}$ on the both sides. After similar considerations for $\widetilde{O}_{4}^{\prime}, \widetilde{O}_{5}^{\prime}$, one can show that the Callan-Symanzik equation (99) is still correct if one writes

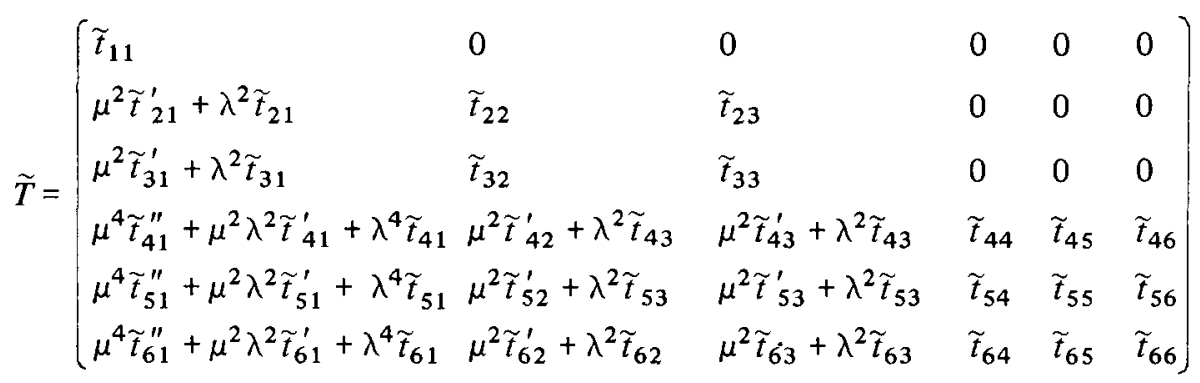

where $\tilde{t}_{i j}, \tilde{t}_{i j}^{\prime}, \tilde{t}_{i j}^{\prime \prime}$ are finite functions of $\alpha$ only. The rest of the arguments in the main text require no major modifications.

\section{References}

[1] K. Wilson, Phys. Rev. 179 (1969) 1499.

[2] T. Kinoshita, J. Math. Phys. 3 (1962) 650;

T.D. Lee and M. Nauenberg, Phys. Rev. 133 (1964) B1549. 
[3] D.J. Gross and F. Wilczek, Phys. Rev. Lett. 30 (1973) 1323; Phys. Rev. D8 (1973) 3633; D9 (1974) 980;

H.D. Politzer, Phys. Rev. Lett. 30 (1973) 1346.

[4] T. Appelquist and J. Carazzone, Phys. Rev. D11 (1975) 2856.

[5] C. Callan, Phys. Rev. D2 (1972) 154;

K. Symanzik, Comm. Math. Phys. 18 (1970) 227.

[6] C. Lee, Ph. D. thesis, chap. II, Stanford University (1975);

M. Gomes and B. Schroer, Phys. Rev. D10 (1974) 3525.

[7] W. Zimmerman, Lectures on elementary particles and quantum field theory, ed., S. Deser, M. Grisaru and H. Pendelton, (M.I.T. Press, Cambridge, Mass., 1971).

[8] J.D. Bjorken and S.D. Drell, Relativistic quantum fields (McGraw-Hill, New York, 1965).

[9] S. Weinberg, Phys. Rev. 118 (1960) 838.

[10] J.A. Dixon and J.C. Taylor, Nucl. Phys. B78 (1974) 552;

H. Kluberg-Stern and J.B. Zuber, Phys. Rev. D12 (1975) 3159;

S.D. Joglekar and B.W. Lee, Ann. of Phys. 97 (1976) 160;

C. Lee, Phys. Rev. D14 (1976) 1078.

[11] S. Weinberg, Phys. Rev. D8 (1973) 3497.

[12] E. Witten, Nucl. Phys. B104 (1976) 445; B122 (1977) 109.

[13] M.A. Shifman, A.I. Vainshtein and V.I. Zakharov, Phys. Rev. D18 (1978) 2583.

[14] C. Lee, unpublished.

[15] B.W. Lee, Chiral dynamics (Gordon and Breach, New York, 1972). 